\title{
WestVirginiaUniversity
}

THE RESEARCH REPOSITORY @ WVU

Graduate Theses, Dissertations, and Problem Reports

2017

\section{On the Matroid Intersection Conjecture}

\author{
Shadisadat Ghaderi
}

Follow this and additional works at: https://researchrepository.wvu.edu/etd

\section{Recommended Citation}

Ghaderi, Shadisadat, "On the Matroid Intersection Conjecture" (2017). Graduate Theses, Dissertations, and Problem Reports. 5665.

https://researchrepository.wvu.edu/etd/5665

This Dissertation is protected by copyright and/or related rights. It has been brought to you by the The Research Repository @ WVU with permission from the rights-holder(s). You are free to use this Dissertation in any way that is permitted by the copyright and related rights legislation that applies to your use. For other uses you must obtain permission from the rights-holder(s) directly, unless additional rights are indicated by a Creative Commons license in the record and/ or on the work itself. This Dissertation has been accepted for inclusion in WVU Graduate Theses, Dissertations, and Problem Reports collection by an authorized administrator of The Research Repository @ WVU.

For more information, please contact researchrepository@mail.wvu.edu. 


\title{
On the Matroid Intersection Conjecture
}

\author{
Shadisadat Ghaderi \\ Dissertation submitted to the \\ Eberly College of Arts and Sciences \\ at West Virginia University \\ in partial fulfillment of the requirements \\ for the degree of
}

Doctor of Philosophy

in

Mathematics

Jerzy Wojciechowski, Ph.D., Chair

Krzysztof Ciesielski, Ph.D.

Elaine Eschen, Ph.D.

John Goldwasser, Ph.D.

Michael Mays, Ph.D.

Department of Mathematics

Morgantown, WV

2017

Keywords: Matroid Intersection, Packing, Covering, Critical Set, Singular Matroids.

Copyright 2017 Shadisadat Ghaderi 


\section{ABSTRACT \\ On the Matroid Intersection Conjecture}

\section{Shadisadat Ghaderi}

In this dissertation, we investigate the Matroid Intersection Conjecture for pairs of matroids on the same ground set, proposed by Nash-Williams in 1990. Originally, the conjecture was stated for finitary matroids only, but we consider it for general matroids and introduce new approaches to attack the conjecture.

The first approach is to consider the situation when it is possible to make a finite modification to the matroids after which the pair satisfies the conjecture. In such a situation we say that the pair has the Almost Intersection Property. We prove that any pair of matroids with the Almost Intersection Property must satisfy the Matroid Intersection Conjecture. Using this result we prove that the Matroid Intersection Conjecture is true in the case when one of the matroids has finite rank and also in the case when one of the matroids is a patchwork matroid.

Our second new approach is inspired by the proof of the general version of König's Theorem for bipartite graphs. That result implies that the Matroid Intersection Conjecture is true for pairs of partition matroids. We develop some new techniques that generalize the critical set approach used in the proof of the countable version of König's Theorem. Our results enable us to prove that the Matroid Intersection Conjecture is true for a pair of singular matroids on a set that is infinitely countable. A matroid is singular when it is a direct sum of matroids such that each term of the sum is a uniform matroid either of rank one or of co-rank one. 


\section{Acknowledgments}

First and foremost, I am most indebted to my supervisor, Dr. Jerzy Wojciechowski, for his continued encouragement and support over these last few years. It is a pleasure to work under his supervision. Without him, this dissertation could not have come about.

I would also like to thank my other committee members: Dr. Krzysztof Ciesielski, Dr. Elaine Eschen, Dr. John Goldwasser, and Dr. Michael Mays, for their help during my studies.

And finally, I would like to thank the Department of Mathematics and Eberly College of Arts and Sciences at West Virginia University for providing me with an excellent study environment and support during my study as a graduate student. 


\section{DEDICATION}

To

My Mother

and

the loving memory of my father and sister Mitra 


\section{Contents}

1 Introduction $\quad 1$

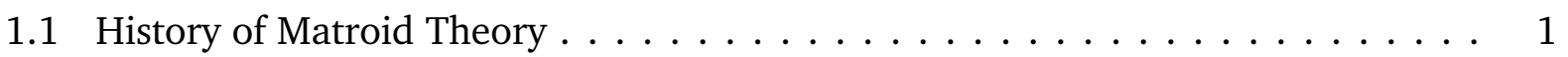

1.2 Matroid Intersection Conjecture . . . . . . . . . . . . . . 2

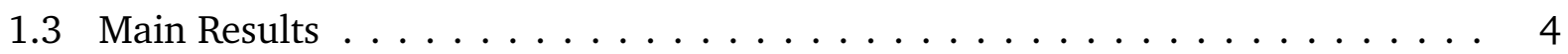

2 Foundations $r$

2.1 Origins of General Matroid $\ldots \ldots \ldots \ldots \ldots \ldots$

2.2 Axiom Systems for General Matroids . . . . . . . . . . . . . . . . . . 10

2.2 .1 Independence Axioms . . . . . . . . . . . . . . . . 11

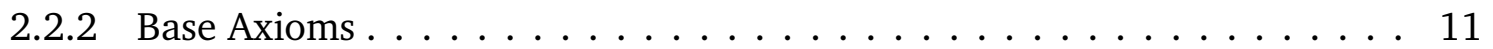

2.2 .3 Closure Axioms . . . . . . . . . . . . . . . . . 12

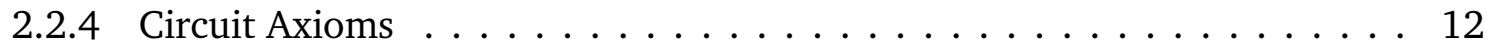

2.2 .5 Rank Axioms . . . . . . . . . . . . . . . . . 13

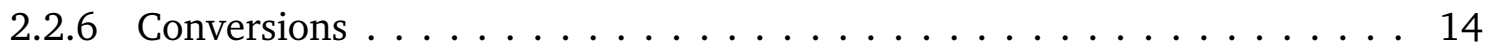

2.2 .7 Spanning Sets . . . . . . . . . . . . . . . . . . . 14

2.2.8 The Circuit Elimination Axiom $\ldots \ldots \ldots \ldots \ldots \ldots$

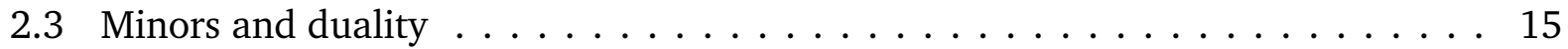

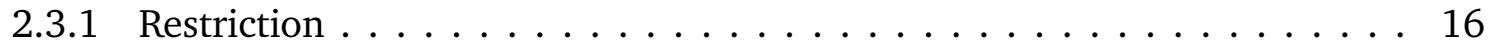

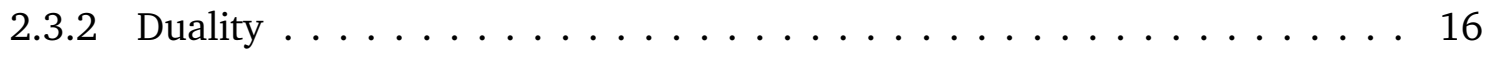

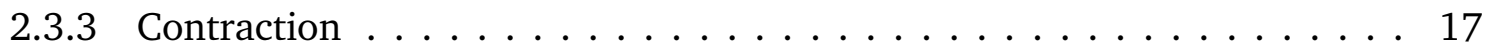


2.4 The Orthogonality Axioms $\ldots \ldots \ldots \ldots \ldots \ldots \ldots$

2.5 Examples . . . . . . . . . . . . . . . . . . . . . 19

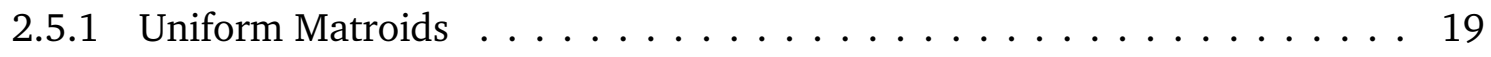

2.5 .2 Cycle and Bond Matroids in Graphs _ . . . . . . . . . . . 20

2.5.3 The Algebraic Cycle Matroid of a Graph . . . . . . . . . . . . . . . 20

2.5 .4 Partition Matroids $\ldots \ldots \ldots \ldots \ldots \ldots \ldots \ldots$

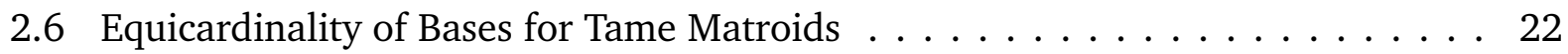

3 Almost Intersection $\quad 24$

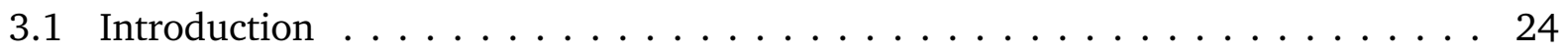

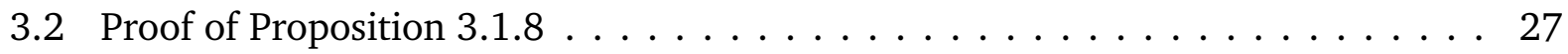

3.3 Proof of Theorems 3.1 .9 and $3.1 .10 \ldots \ldots \ldots \ldots 29 \ldots \ldots \ldots$

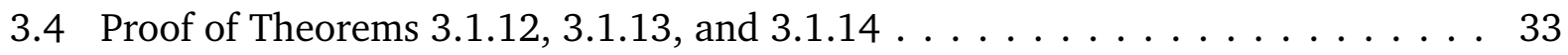

4 Critical Sets $\quad 36$

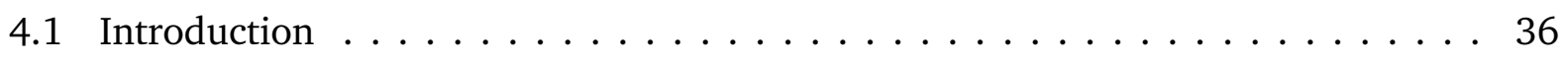

4.2 Proof of Theorem $4.1 .1 \ldots \ldots \ldots \ldots$

4.3 Critical Sets for Matroids $\ldots \ldots \ldots \ldots \ldots \ldots$

4.4 Proof of Main Results . . . . . . . . . . . . . . . . . . . . . . 43

4.4.1 Infinite Exchange Chain $\ldots \ldots \ldots \ldots \ldots \ldots \ldots$

4.4.2 Proof of Theorems 4.1.9, 4.1.10, and Corollary 4.1.11 . . . . . . . . 47

5 Matroid Intersection Conjecture for Singular Matroids 59

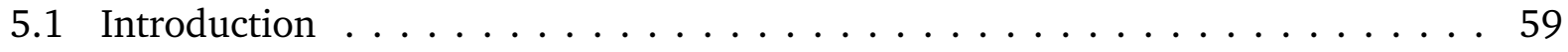

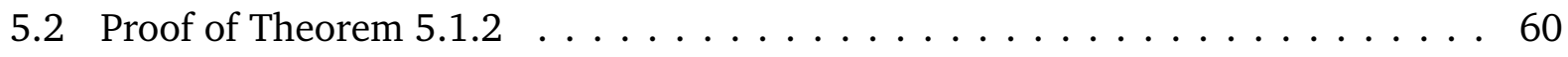

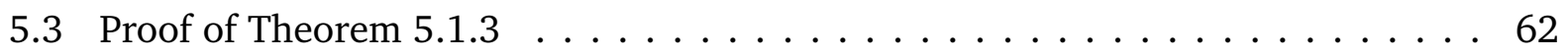

5.4 Proof of Theorem 5.1.4 and Corollary $5.1 .5 \ldots \ldots \ldots \ldots \ldots$ 
6 Conclusion and Future Work $\quad 92$

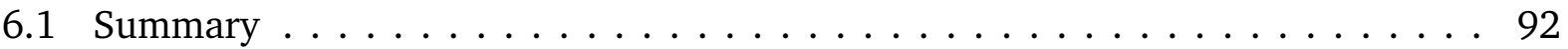

6.2 Statements Equivalent to the Matroid Intersection Conjecture . . . . . . . . . . 93

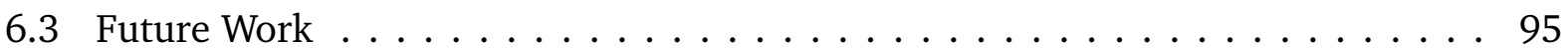




\section{Chapter 1}

\section{Introduction}

\subsection{History of Matroid Theory}

The theory of general matroids originates from two sources. One is the theory of finite matroids introduced by Whitney [38] in 1935, that was also independently discovered by Nakasawa, whose work was forgotten for many years (see [30]). The other source is the result by Sierpiński [35] (see also [36]) in 1945, on duality in Fréchet $V$-spaces without isolated points (unaware of Whitney's work). General matroids were most often defined like finite matroids, by adding the following axiom:

(I4) An infinite set is independent as soon as all its finite subsets are independent.

One of the destructive consequence of (I4) is that it destroys duality, which is one of the key aspects of finite matroid theory. As a consequence, Rado asked for the expansion of a theory of general matroids with duality in 1966 ([34], Problem P531). Rado's challenge began some serious research and activity in the late 1960s (see for example [31]), in which many mathematicians proposed various possible approaches to general matroids. In 1969, Higgs [27] combined the theory of finite matroids with Sierpiński's result to build a theory of general matroids, which he called B-matroids. Oxley [31] showed that B-matroids have the properties of a suitable extension and answered Rado's problem. In 2008, Bruhn, Diestel, Kriesell, 
Pendavingh and Wollan [19] rediscovered the concept of a general matroid equivalent to Bmatroids of Higgs. They introduced five equivalent axiomatizations for general matroids, providing a foundation on which a theory of general matroids with duality can be built. They proposed these equivalent sets of matroid axioms, in terms of independent sets, bases, circuits, closure, and rank, that make duality possible. In this dissertation, we follow the axioms introduced in [19].

\subsection{Matroid Intersection Conjecture}

In the development of general matroid theory, there have been a number of conjectures about how to possibly extend the standard and classical theorems of finite matroid theory to infinite sets. These include the matroid intersection theorem which is a classical result in finite matroid theory.

The well-known finite matroid intersection theorem of Edmonds [22] states that for any two finite matroids $M$ and $N$, the size of a biggest common independent set is equal to the minimum of the rank sum $r_{M}\left(E_{M}\right)+r_{N}\left(E_{N}\right)$, where the minimum is taken over all partitions $E=E_{M} \sqcup E_{N}$. Here rank of a matroid refers to the number of elements of a base of the matroid. In extending this statement to the infinite case, Nash-Williams [5] proposed the following in 1990.

Conjecture 1.2.1 (The Matroid Intersection Conjecture [5]). Any two matroids $M$ and $N$ on a common set $E$ have a common independent set I admitting a partition $I=J_{M} \sqcup J_{N}$ such that $\mathrm{cl}_{M}\left(J_{M}\right) \cup \mathrm{cl}_{N}\left(J_{N}\right)=E$.

Here $\operatorname{cl}_{M}(X)$ denotes the closure of a set $X$, in a matroid $M$ that consists of the set $X$ and the elements spanned by $X$ in $M$.

When Nash-Williams first made this conjecture in 1990, he only had finitary matroids i.e., all of whose circuits are finite, in mind, because at that time general matroids were considered as finitary matroids. 
This dissertation is focused on the Matroid Intersection Conjecture 1.2.1 one of important open problem in the theory of general matroids. As a motivation for working on this conjecture, we can point to the infinite Menger theorem. The infinite Menger theorem was conjectured by Erdős in the 1960s and proved recently by Aharoni and Berger [7]. It states that if $A$ and $B$ are sets of vertices in a (possibly infinite) graph $G$, then there exists a family $P$ of disjoint $A-B$-paths and a separating set which consists of exactly one vertex from each path in $P$. Due to the complexity of the only known proof of this theorem, the investigation of a matroidal proof of the infinite Menger theorem attracts attention among researchers. In [7] this is shown; specifically, it is proved that the Matroid Intersection Conjecture 1.2.1 for finitary matroids implies the infinite Menger theorem.

Remark 1.2.2. The Matroid Intersection Conjecture 1.2.1 is known to be true for the following cases:

- When $M$ is finitary and $N$ is a countable direct sum of finite rank matroids ([5]). (We call a matroid direct sum if the ground set can be partitioned so that each circuit is a subset of one part, and we call a matroid finite rank if the cardinality of its bases is finite).

- When $M$ is finitary and $N$ is co-finitary ([7]). (We call a matroid finitary if all its circuits are finite and co-finitary if its dual is finitary).

- When $M$ is nearly finitary and $N$ is the dual of a nearly finitary matroid ([7]). (We call a matroid nearly finitary if by removing finitely many elements from any subset that contains no finite circuit, we get an independent set).

- When $M$ and the dual of $N$ have only countably many circuits ([12]).

- When $M$ and $N$ are tame matroids which have a common decomposition by 2-separations into finite parts ([11]). (We call a matroid tame if the intersection of any of its circuit with any of its co-circuit is finite). 
- When $M$ and $N$ are partition matroids. We show in Section 4.2 that this case follows from Theorem 4.1.2. (Theorem 4.1.2 was proved in [2] using a deep result from [3]).

\subsection{Main Results}

The content of this dissertation will be published in two papers: [14] and [23]. The content of [14] is described in Chapter 3 and the content of [23] is described in Chapters 4 and 5.

For the rest of this section assume that $M$ and $N$ are matroids on a common ground set $E$. In [12], Packing/Covering Property is introduced i.e. $(M, N)$ has the Packing/Covering Property if and only if there exists a partition $E=P \sqcup C$ such that $(M, N)$ restricted to $P$ has a packing and $(M, N)$ contracted to $C$ has a covering. The pairwise Packing/Covering conjecture [12] says that any pair of matroids has the Packing/Covering Property. It is shown in [12] that the Matroid Intersection conjecture and the pairwise Packing/Covering conjecture are equivalent.

In the coming several chapters, we will present the following main results.

(1) In Chapter 3, we introduce Almost Intersection Property (see Definition 3.1.5), Almost Packing/Covering Property (see Definition 3.1.6), and Packing/Covering Property modulo a finite set (see Definition 3.1.7) for a pair of matroids $(M, N)$. All of those concepts, each in a different way, convey the idea that after a finite modification the pair of matroids $(M, N)$ satisfies the original property.

Then we prove the following main results.

Proposition. 3.1.8. $(M, N)$ has the Almost Intersection Property if and only if $\left(M, N^{*}\right)$ has the Packing/Covering Property modulo a finite set, where $N^{*}$ is the dual matroid of $N$.

Theorem. 3.1.9. If $(M, N)$ has the Almost Intersection Property, then it satisfies the Matroid Intersection Conjecture.

Theorem. 3.1.10. The following are equivalent.

(a) $(M, N)$ has the Packing/Covering Property. 
(b) $(M, N)$ has the Almost Packing/Covering Property.

(c) $(M, N)$ has the Packing/Covering Property modulo a finite subset of $E$

Using these results, we prove that the Matroid Intersection Conjecture 1.2.1 is true for the following cases:

Theorem. 3.1.12. If $M$ has finite rank and $N$ is arbitrary, then $(M, N)$ satisfies the Matroid Intersection Conjecture.

Theorem. 3.1.14. If $M$ is patchwork and $N$ is arbitrary, then $\left(M, N^{*}\right)$ satisfies the Matroid Intersection Conjecture.

Furthermore, we provide an alternative proof that the Matroid Intersection Conjecture 1.2.1 is true for the following case:

Theorem. 3.1.13. If $M$ and $N$ are nearly finitary, then $\left(M, N^{*}\right)$ satisfies the Matroid Intersection Conjecture.

(2) In Chapter 4, we develop new techniques and prove results about general matroids that we plan to use as tools to attack the Matroid Intersection Conjecture 1.2.1. We will use these techniques and results in Chapter 5 to prove the Matroid Intersection Conjecture 1.2.1 for a particular family of matroids. In Chapter 4, we introduce essential element. We say $a \in E$ is essential for $(M, N)$ if and only if $(M, N)$ has a covering and $(M, N) /\{a\}$ has no covering. Then, we introduce the special covering (see Definition 4.4.2). Finally, we introduce critical sets for pairs of matroids: We say $A \subseteq E$ is critical for $(M, N)$ if and only if $\left(M \uparrow_{A}, N \uparrow_{A}\right)$ has a covering and each covering $(I, J)$ for $\left(M \uparrow_{A}, N \uparrow_{A}\right)$ is also a packing. Then we prove the following main results.

Theorem. 4.1.9. Let $(I, J)$ be a special covering and $a \in E$ essential for $(M, N)$. Then there exists a critical set $A \subseteq E$ for $(M, N)$ such that $a \in A$.

Theorem. 4.1.10. If $(M, N)$ has a covering, then there exists a maximal critical set. 
Corollary. 4.1.11. Let $(I, J)$ be a special covering and $E^{\prime} \subseteq E$ be such that every $a \in E^{\prime}$ is essential for $(M, N)$. Then there exists a critical set $K \subseteq E$ such that $E^{\prime} \subseteq K$.

(3) In Chapter 5, we introduce the concept blockage. We say that $(M, N)$ has a blockage if and only if

- there exists a critical set $K \subseteq E$ for $(M, N)$ and $a \in E \backslash K$ such that $a \in \operatorname{cl}_{M}(K)$ and $a \in \mathrm{cl}_{N}(K)$.

Then we prove the following main result which concerns arbitrary matroids.

Theorem. 5.1.2. Suppose for matroids $M$ and $N$ on a common set $E$, the followings are equivalent:

(a) $(M, N)$ has a covering.

(b) $(M, N)$ has no blockage.

Then $(M, N)$ has the Packing/Covering Property.

Then we introduce singular matroids. We say that matroids $M$ and $N$ on a common set are singular if and only if each one is a direct sum of matroids such that each term of the sum is a uniform matroid either of rank one or of co-rank one (see Definition 5.3.1). Then we prove the following main results which concern singular matroids.

Theorem. 5.1.3. If $M$ and $N$ are singular, then there exists a maximal critical set for $M$ and $N$.

Theorem. 5.1.4. Let $M$ and $N$ be singular matroids on an infinite countable set $E$. Then the followings are equivalent:

(a) $(M, N)$ has a covering.

(b) $(M, N)$ has no blockage. 
Finally we show that Theorem 5.1.4 and Theorem 5.1.2 imply that the Matroid Intersection Conjecture is true for singular matroids.

Corollary. 5.1.5. If $M$ and $N$ are singular on an infinite countable set $E$, then $M$ and $N$ satisfy the Matroid Intersection Conjecture. 


\section{Chapter 2}

\section{Foundations}

In this chapter, we provide the essential background that is required for the coming chapters. Any matroid terminology not explained below is taken from Oxley [32] and [19]. We also follow these two notations $\mathbb{N}=\{1,2, \ldots\}$ and $\mathbb{N}_{0}=\mathbb{N} \cup\{0\}$. In Section 1 , we introduce general matroids using topology related terminology and give connections with finite matroids and Sierpiński result on the duality for in Fréchet $V$-spaces with no isolated points. In Section 2, we give more standard definitions of general matroids following [19]. We state axiom systems introduced in [19] for general matroids, and define general matroids as set systems satisfying the independence axioms. In Section 3, we define the dual matroid and two important minor matroids: restriction and contraction. In Section 4, we state the orthogonality axioms. Section 5 is devoted to examples of general matroids. In Section 6, we state the results and proof of equicardinality of bases of tame matroids.

\subsection{Origins of General Matroid}

Definition 2.1.1. Let $E$ be a set and $\mathscr{P}(E)$ the family of all subsets of $E$. A pre-closure operation on $E$ is a function $\mathrm{cl}: \mathscr{P}(\mathrm{E}) \rightarrow \mathscr{P}(\mathrm{E})$ such that satisfies the followings:

(CL1) For all $X \subseteq E$ we have $X \subseteq \operatorname{cl}(X)$. 
(CL2) For all $X \subseteq Y \subseteq E$ we have $\operatorname{cl}(X) \subseteq \operatorname{cl}(Y)$.

Definition 2.1.2. Let $\mathrm{cl}: \mathscr{P}(\mathrm{E}) \rightarrow \mathscr{P}(\mathrm{E})$ be a pre-closure operation on $E$. We say that $x \in E$ is a loop if and only if $x \in \operatorname{cl}(\emptyset)$ and is a co-loop (isolated point) if and only if $x \notin \operatorname{cl}(E \backslash\{x\})$.

Definition 2.1.3. Let $\mathrm{cl}: \mathscr{P}(\mathrm{E}) \rightarrow \mathscr{P}(\mathrm{E})$ be a pre-closure operation on $E$. We say that the set $I \subseteq E$ is cl-independent if and only if $x \notin \operatorname{cl}(I \backslash\{x\})$ for all $x \in I$.

Definition 2.1.4. Let $\mathrm{cl}: \mathscr{P}(\mathrm{E}) \rightarrow \mathscr{P}(\mathrm{E})$ be a pre-closure operation on $E, A \subseteq E$, and $a \in E$. We define $\mathrm{cl}^{*}: \mathscr{P}(\mathrm{E}) \rightarrow \mathscr{P}(\mathrm{E})$ by

$$
a \in \mathrm{cl}^{*}(A) \quad \text { if and only if } \quad a \in A \text { or } a \in \operatorname{cl}(E \backslash(A \cup\{a\})) \text {. }
$$

Observe that the function $\mathrm{cl}^{*}$ also satisfies (CL1) and (CL2), and hence $\mathrm{cl}^{*}$ is also a preclosure operation on $E$. We say that $\mathrm{cl}^{*}$ is the pre-closure operation dual to $\mathrm{cl}$.

Definition 2.1.5. Let $E$ be a set. A closure operation on $E$ is a pre-closure operation on $E$ such that it also satisfies the following:

(CL3) For all $X \subseteq E$ we have $\operatorname{cl}(\operatorname{cl}(X))=\operatorname{cl}(X)$.

Definition 2.1.6. A pair $(E, \mathscr{I})$ is called a finite matroid where $E$ is a finite set and $\mathscr{I}$ is the set of all cl-independent sets for some pre-closure operation $\mathrm{cl}$ on $E$ such that $\mathrm{cl}$ and $\mathrm{cl}^{*}$ are both closure operations.

Definition 2.1.7. A pair $(E, \mathrm{cl})$ is called a Fréchet $V$-space where $E$ is a set and $\mathrm{cl}$ is a closure operation on $E$ such that $\operatorname{cl}(\emptyset)=\emptyset$ (see chapter $1[36]$ ).

Sierpiński proved the following result (apparently without knowing anything about Whitney's work on matroids).

Theorem 2.1.8 ([35]). If $(E, \mathrm{cl})$ is a Fréchet V-space with no isolated points, then $\left(E, \mathrm{cl}^{*}\right)$ is also a Fréchet V-space with no isolated points. 
To define matroids (general matroids) we need the followings:

- Let $E$ be a set and $\mathscr{I} \subseteq \mathscr{P}(\mathrm{E})$. We say that $I \in \mathscr{I}$ is maximal in $\mathscr{I}$ if and only if for every $J \in \mathscr{I}$ the inclusion $I \subseteq J$ implies that $I=J$.

- Let $\mathscr{I} \subseteq \mathscr{P}(\mathrm{E})$. The following statement describes a possible property of $\mathscr{I}$.

(M) Whenever $I \subseteq X \subseteq E$ and $I \in \mathscr{I}$, the set $\left\{I^{\prime} \in \mathscr{I} ; I \subseteq I^{\prime} \subseteq X\right\}$ has a maximal element.

Definition 2.1.9. A pair $(E, \mathscr{I})$ is called a matroid where $E$ is a set and $\mathscr{I}$ is the set of all cl-independent sets for a closure operation $\mathrm{cl}$ on $E$ such that in addition to (CL1), (CL2), and (CL3) it also satisfies the followings:

(CL4) For all $Z \subseteq E$ and $x, y \in E$, if $y \in \operatorname{cl}(Z \cup\{x\}) \backslash \operatorname{cl}(Z)$ then $x \in \operatorname{cl}(Z \cup\{y\})$.

(CLM) The set $\mathscr{I}$ satisfies (M).

Note that if $E$ is finite, then Definition 2.1.9 is equivalent to Definition 2.1.6 (since (CL4) implies that $\mathrm{cl}^{*}$ is a closure operation). Note also that if matroid $(E, \mathscr{I})$ has no loops and no co-loops, then $(E, \mathrm{cl})$ is a in Fréchet $V$-space with no isolated points (recall that $\mathrm{cl}$ is a closure operation on $E$ such that $\mathscr{I}$ is the set of all cl-independent sets).

\subsection{Axiom Systems for General Matroids}

In this section, we state [19] five systems of axioms for general matroids. They are stated, respectively, in terms of independent sets, bases, closure, circuits, and rank. Theses axioms allow infinite circuits, which leads to a theory of matroids that is not necessary finitary matroids. On the other side, in the case that circuits are finite, they default to finitary matroids. Therefore, these axioms generate a theory of matroids which include the family of finitary matroids. Duality will work as familiar from finite matroids: the co-bases are the complements of bases, and there are well-defined and dual operations of contraction and deletion extending the familiar finite operations. In developing these axioms, one objective was that every independent set 
to extend to a maximal one, and the other objective was that every dependent set to contain a minimal one. Moreover, they wanted to have the property that every independent set extends to a maximal one, inside any restriction $X \subseteq E$. To state these axioms, we recall (M): Let $\mathscr{I} \subseteq \mathscr{P}(\mathrm{E})$. The following statement describes a possible property of $\mathscr{I}$.

(M) Whenever $I \subseteq X \subseteq E$ and $I \in \mathscr{I}$, the set $\left\{I^{\prime} \in \mathscr{I} ; I \subseteq I^{\prime} \subseteq X\right\}$ has a maximal element.

\subsubsection{Independence Axioms}

Let $E$ be a set and $\mathscr{P}(E)$ the family of all subsets of $E$. The following statements about a set $\mathscr{I} \subseteq \mathscr{P}(\mathrm{E})$ are independence axioms:

(I1) $\phi \in \mathscr{I}$.

(I2) For $I \in \mathscr{I}$ and $I^{\prime} \subseteq I$ we have $I^{\prime} \in \mathscr{I}$.

(I3) If $I, J \in \mathscr{I}$ with $I$ maximal and $J$ not maximal, then there exists an $x \in I \backslash J$ such that $J \cup\{x\} \in \mathscr{I}$.

(IM) $\mathscr{I}$ satisfies (M).

Definition 2.2.1. When a set $\mathscr{I} \subseteq \mathscr{P}$ (E) satisfies the independence axioms, we call the pair $M=(E, \mathscr{I})$ a matroid on $E$. We then call every element of $\mathscr{I}$ an independent set, every element of $\mathscr{P}(\mathrm{E}) \backslash \mathscr{I}$ a dependent set, the maximal independent sets bases, and and the minimal dependent sets circuits. The function $\mathrm{cl}_{M}: \mathscr{P}(\mathrm{E}) \rightarrow \mathscr{P}(\mathrm{E})$

$$
\mathrm{cl}_{\mathrm{M}}(A)=A \cup\{a \in E: \text { there exists a circuit } C \text { of } M \text { such that } a \in C \subseteq A \cup\{a\}\}
$$

will be called the closure operator on $\mathscr{P}($ E) associated with $\mathscr{I}$.

\subsubsection{Base Axioms}

The following statements about a set $\mathscr{B} \subseteq \mathscr{P}(\mathrm{E})$ are base axioms: 
(B1) $\mathscr{B} \neq \emptyset$.

(B2) For $B_{1}, B_{2} \in \mathscr{B}$ and $x \in B_{1} \backslash B_{2}$, there is an element $y \in B_{2} \backslash B_{1}$ such that $B_{1} \cup\{y\} \backslash\{x\} \in \mathscr{B}$.

(BM) The set $\mathscr{I}$ of all subsets of elements of $\mathscr{B}$ satisfies (M).

Definition 2.2.2. Let $M=(E, \mathscr{I})$ be a matroid. A base of $M$ is a maximal element of $\mathscr{I}$. The family of all bases of $\mathrm{M}$ is denoted by $\mathscr{B}$.

Remark. Note that it follows from (I3) that any element of $\mathscr{I}$ is contained in a base of $M$.

\subsubsection{Closure Axioms}

The following statements about a function $\mathrm{cl}: \mathscr{P}(\mathrm{E}) \rightarrow \mathscr{P}(\mathrm{E})$ are closure axioms:

(CL1) For all $X \subseteq E$ we have $X \subseteq \operatorname{cl}(X)$.

(CL2) For all $X \subseteq Y \subseteq E$ we have $\operatorname{cl}(X) \subseteq \operatorname{cl}(Y)$.

(CL3) For all $X \subseteq E$ we have $\operatorname{cl}(\operatorname{cl}(X))=\operatorname{cl}(X)$.

(CL4) For all $Z \subseteq E$ and $x, y \in E$, if $y \in \operatorname{cl}(Z \cup\{x\}) \backslash \operatorname{cl}(Z)$ then $x \in \operatorname{cl}(Z \cup\{y\})$.

(CLM) The set $\mathscr{I}$ of all cl-independent sets satisfies (M). These are the sets $I \subseteq E$ such that $x \notin \operatorname{cl}(I \backslash\{x\})$ for all $x \in I$.

Sets of the form $\operatorname{cl}(X)$ are called closed sets. Thus by (CL3) a subset $X$ of $E$ is closed if and only if $X=\operatorname{cl}(X)$. A subset $X$ of $E$ is said to be spanning if $\operatorname{cl}(X)=E$.

Remark. Note that the the closure operator $\mathrm{cl}_{M}: \mathscr{P}(\mathrm{E}) \rightarrow \mathscr{P}(\mathrm{E})$ defined in Definition 2.2.1 satisfies the closure axioms.

\subsubsection{Circuit Axioms}

Definition 2.2.3. Let $\mathscr{C} \subseteq \mathscr{P}(\mathrm{E})$. Define $\mathrm{cl}_{\mathscr{C}}: \mathscr{P}(\mathrm{E}) \rightarrow \mathscr{P}(\mathrm{E})$ by

$a \in \operatorname{cl}_{\mathscr{C}}(A)$ if and only if $a \in A$ or there is some $C \in \mathscr{C}$ such that $a \in C \subseteq A \cup\{a\}$. 
We call $\mathrm{cl}_{\mathscr{C}}$ closure-like operation induced by a family $\mathscr{C}$.

The following statements about a set $\mathscr{C} \subseteq \mathscr{P}$ (E) are circuit axioms:

(C1) $\emptyset \notin \mathscr{C}$.

(C2) No element of $\mathscr{C}$ is a subset of another.

(C3) The operation $\mathrm{cl}_{\mathscr{C}}$ is transitive.

(CM) The set $\mathscr{I}$ of all $\mathscr{C}$-independent sets satisfies (M). These are the sets $I \subseteq E$ such that $C \nsubseteq I$ for all $C \in \mathscr{C}$.

The axiom (C3) is called the circuit elimination axiom. If $\{e\}$ is a circuit, then it is called a loop.

\subsubsection{Rank Axioms}

The set of all pairs $(A, B)$ such that $B \subseteq A \subseteq E$ will be denoted by $(\mathscr{P}(\mathrm{E}) \times \mathscr{P}(\mathrm{E}))_{\subseteq}$. The following statements about a function $r:(\mathscr{P}(\mathrm{E}) \times \mathscr{P}(\mathrm{E}))_{\subseteq} \rightarrow \mathbb{N}_{0} \cup\{\infty\}$ are rank axioms:

(R1) For all $B \subseteq A \subseteq E$ we have $r(A, B) \leq|A \backslash B|$.

(R2) For all $A, B \subseteq E$ we have $r(A, A \cap B) \leq r(A \cup B, B)$.

(R3) For all $C \subseteq B \subseteq A \subseteq E$ we have $r(A, C) \leq r(A, B)+r(B, C)$.

(R4) For all families $\left(A_{\gamma}\right)$ and $B$ such that $B \subseteq A_{\gamma} \subseteq E$ and $r\left(A_{\gamma}, B\right)=0$ for all $\gamma$, we have $r(A, B)=0$ for $A:=\bigcup_{\gamma} A_{\gamma}$.

(RM) The set $\mathscr{I}$ for all $r$-independent sets satisfies (M). These are the sets $I \subseteq E$ such that $r(I, I \backslash\{x\})>0$ for all $x \in I$. 


\subsubsection{Conversions}

Let $\mathscr{I}$ be the family of independent sets of a matroid, then the family of maximal independent sets is the set of bases of the same matroid, the family $\mathscr{C}$ of minimal dependent sets is the set of circuits of the same matroid, the operator $\mathrm{cl}: \mathscr{P}(\mathrm{E}) \rightarrow \mathscr{P}(\mathrm{E})$

$$
\operatorname{cl}(A)=A \cup\{a \notin A \text { : there exists some } I \in \mathscr{I} \text { with } I \subseteq A \text { and } I \cup\{a\} \notin \mathscr{I}\}
$$

is the closure operator of the same matroid.

In the other direction, if $\mathscr{B}$ is the family of bases of a matroid, then the family of subsets of elements of $\mathscr{B}$ is the family of independent sets of the same matroid. If $\mathscr{C}$ is the family of circuits of a matroid, then the subsets of $E$ that include no element of $\mathscr{C}$ form the family of independent sets of the same matroid. If $\mathrm{cl}$ is the closure operator of a matroid, then those subsets $I \subseteq E$ that has no $e \in I$ with $e \in \operatorname{cl}(I \backslash\{e\})$ form the family of independent sets of the same matroid. The closure operator also can be defined in the terms of the circuits: if $\mathscr{C}$ is the family of circuits of a matroid, then $\mathrm{cl}_{\mathscr{C}}: \mathscr{P}(\mathrm{E}) \rightarrow \mathscr{P}(\mathrm{E})$

$$
\operatorname{cl}_{\mathscr{C}}(A)=A \cup\{a \notin A: \text { there exists some circuit } C \in \mathscr{C} \text { such that } a \in C \subseteq A \cup\{a\}\}
$$

is the closure operator of the same matroid.

By these conversions, we can see that all of these axioms are in fact different descriptions of the same sort of mathematical object. If $M$ is a matroid, then we will refer to the set of independent sets of $M$ as $\mathscr{I}$, the set of bases of $M$ as $\mathscr{B}$, the set of circuits of $M$ as $\mathscr{C}$, the closure operator of $M$ as $\mathrm{cl}_{\mathrm{M}}$, and the rank function of $M$ as $r_{M}$.

\subsubsection{Spanning Sets}

If $M$ is a matroid on a ground set $\mathrm{E}$, then a subset $I \subseteq E$ is a spanning set for the matroid $M$ if and only if $\mathrm{cl}_{\mathrm{M}}(I)=E$. By this definition, it is clear that an independent set is a base if and 
only if it is a spanning set. Therefore any set that contains a base is a spanning set. Conversely, if $S$ is a spanning set and $B$ is a base of $M$, then $S \subseteq \operatorname{cl}(B)$ and so

$$
\operatorname{cl}(B) \supseteq \operatorname{cl}(\operatorname{cl}(B)) \supseteq \operatorname{cl}(S)=E .
$$

This implies that $B$ is both independent and spanning, and hence is a base. Therefore the bases are precisely the minimal spanning sets.

\subsubsection{The Circuit Elimination Axiom}

The circuit elimination axiom (C3) (introduced in Section 2.2.4) is an extension of the usual circuit elimination axiom for finite matroids (C3'):

(C3') Circuit elimination axiom. For any distinct $C_{1}, C_{2} \in \mathscr{C}$ and any $c \in C_{1} \cap C_{2}$, there exists some $C_{3} \in \mathscr{C}$ such that

$$
C_{3} \subseteq\left(C_{1} \cup C_{2}\right) \backslash\{c\} .
$$

Specially, axiom (C3) implies that adding an element to a base creates at most one circuit.

Lemma 2.2.4 ([19]). Let $\mathscr{C}$ be a set of subsets of E satisfying (C3'), $x \in E$, and I a $\mathscr{C}$-independent set. Then there is at most one nonempty $C \in \mathscr{C}$ with $C \subseteq I \cup\{e\}$.

In particular, if $B$ is a base and $e \notin B$, then there exists a unique circuit $C_{e}^{B}$ with

$$
e \in C_{e}^{B} \subseteq B \cup\{e\} .
$$

This circuit is called the fundamental circuit of $e$ with respect to $B$.

\subsection{Minors and duality}

In this section we state just enough about general matroids $M=(E, \mathscr{I})$ to enable us in the coming chapters to deduce the main results introduced in Section 1.3. On the way, we define 
duality, restriction, and contractions. For more properties of general matroids see for example [20].

\subsubsection{Restriction}

The following is proved in [19].

Theorem 2.3.1 ([19]). If $M=(E, \mathscr{I})$ is a matroid, $X \subseteq E$, and $\mathscr{I}^{\prime}=\mathscr{I} \cap \mathscr{P}(X)$, then $\left(X, \mathscr{I}^{\prime}\right)$ is also a matroid.

This matroid is called the restriction of $M$ to $X$ and denote it by $M \uparrow_{X}$. Bases of $M \uparrow_{X}$ are maximal independent subsets of $X$. For any set $Q$, the matroid $M \uparrow_{E \backslash Q}$ is denoted by $M \backslash Q$ and is said to be obtained from $M$ by deleting $Q$. The following identities are easily verified:

- $\mathscr{C}\left(M \uparrow_{X}\right)=\mathscr{C}(M) \cap X$

- $\mathrm{cl}_{\mathrm{M}_{\mathrm{X}}}(Y)=\mathrm{cl}_{\mathrm{M}}(Y) \cap X$.

- $M \backslash Q_{1} \backslash Q_{2}=M \backslash Q_{2} \backslash Q_{1}=M \backslash\left(Q_{1} \cup Q_{2}\right)$.

\subsubsection{Duality}

The following is proved in [19].

Theorem 2.3.2 ([19]). Let $M=(E, \mathscr{I})$ be a matroid and

$$
\mathscr{I}^{*}=\left\{I^{*} \subseteq E \text { : there is a } B \in \mathscr{B} \text { such that } I^{*} \cap B=\emptyset\right\}
$$

Then $M^{*}=\left(E, \mathscr{I}^{*}\right)$ is also a matroid.

The matroid $M^{*}$ is called the dual matroid of $M$. Let

$$
\mathscr{B}^{*}:=\left\{B^{*} \subseteq E: E \backslash B^{*} \in \mathscr{B}\right\} .
$$


Then $\mathscr{B}^{*}$ is the set of bases of matroid $M^{*}$. Clearly $M^{* *}=M$. Independent sets of $M^{*}$ are called co-independent subsets of $M$. Similarly, bases, circuits, loops, the closure operator, closed sets, spanning sets of $M^{*}$ are called respectively co-bases, co-circuits, co-loops, the co-closure operator, co-closed sets, co-spanning sets of $M$.

Let $B$ be a base of matroid $M=(E, \mathscr{I})$ and $a \in E \backslash B$. Then the set

$$
C=\{b \in B \cup\{a\}: B \cup\{a\} \backslash\{b\} \in \mathscr{I}\}
$$

is the unique circuit of $M$ containing $a$ and contained in $B \cup\{a\}$. It is called the fundamental circuit of $a$ with respect to $B$. If $B$ is a base of $M$ and $e \in B$, then the fundamental circuit of $e$ with respect to the complement of $B$ in $M^{*}$ is denoted by $D_{e}^{B}$, and called the fundamental co-circuit of $e$ with respect to $B$.

Theorem 2.3.3 ([19]). A circuit and a co-circuit of a matroid never meet in exactly one element.

\subsubsection{Contraction}

Contraction is the dual operation to restriction: if $M$ is a matroid with ground set $E$ and $X \subseteq E$, then the matroid $\left(\left.M^{*}\right|_{X}\right)^{*}$ is called the contraction of $M$ to $X$ and is denoted by $M . X$. If $P$ is any set then the matroid $M / P=M .(E \backslash P)$ is said to be obtained from $M$ by contracting $P$. A matroid $N$ is a minor of a matroid $M$ if it is a matroid that can be obtained from $M$ by a sequence of contractions and restrictions.

The following characterization of the contraction are taken from [19]:

Lemma 2.3.4. Let $M=(E, \mathscr{I})$ be a matroid and $X \subseteq E$. Then for every $I \subseteq X$, the following conditions are equivalent:

1. I is independent in M.X.

2. For every $J$ that is independent in $M \backslash X$ we have $I \cup J \in \mathscr{I}$.

3. There is a base $B$ of $M \backslash X$ such that $I \cup B \in \mathscr{I}$. 
Lemma 2.3.5. Let $M=(E, \mathscr{I})$ be a matroid, $X \subseteq E$, and $B \subseteq E \backslash X$. Then the following are equivalent:

1. B is a base of M.X.

2. There is a base $B^{\prime}$ of $M \backslash X$ such that $B \cup B^{\prime}$ is a base of $M$.

3. For any base $B^{\prime}$ of $M \backslash X$, the set $B \cup B^{\prime}$ is a base of $M$.

It is not easy to characterize the circuits of $M . X$, but we have the following.

Lemma 2.3.6. $\mathscr{C}(M . X) \subseteq \mathscr{C}(M) . X$.

Corollary 2.3.7. Let $M=(E, \mathscr{I})$ be a matroid, and $P$ and $Q$ be disjoint sets. Then

$$
M / P \backslash Q=M \backslash Q / P .
$$

\subsection{The Orthogonality Axioms}

The orthogonality axioms are as follows, where $\mathscr{C}$ and $\mathscr{D}$ are sets of subsets of a set $E$ (intended to be the sets of circuits of some matroid and of its dual, respectively).

(C1) $\emptyset \notin \mathscr{C}$.

(C2) No element of $\mathscr{C}$ is a subset of another.

$(\mathrm{C} 1 *) \emptyset \notin \mathscr{D}$.

(C2*) No element of $\mathscr{D}$ is a subset of another.

(O1) $|C \cap D| \neq 1$ for all $C \in \mathscr{C}$ and $D \in \mathscr{D}$.

(O2) For all partitions $E=P \sqcup Q \sqcup\{e\}$ either $P \cup\{e\}$ includes an element of $\mathscr{C}$ through $e$ or $Q \cup\{e\}$ includes an element of $\mathscr{D}$ through $e$. 
(O3) For every $C \in \mathscr{C}, e \in C$, and $X \subseteq E$ there is some $C_{\min } \in \mathscr{C}$ with $e \in C_{\min } \subseteq X \cup C$ such that $C_{\min } \backslash X$ is minimal.

(O3*) For every $D \in \mathscr{D}, e \in D$, and $X \subseteq E$ there is some $D_{\min } \in \mathscr{D}$ with $e \in D_{\min } \subseteq X \cup D$ such that $D_{\min } \backslash X$ is minimal.

The axiom (IM) says that there are bases in all minors. Similarly, the axiom (O3) says that there are circuits in all minors.

The main result of this section is the following proved in [19].

Theorem 2.4.1 ([19]). Let $E$ be a countable set and $\mathscr{C}, \mathscr{D} \subseteq \mathscr{P}(E)$.

Then $\mathscr{C}$ is the set of circuits of a matroid and $\mathscr{D}$ is the set of co-circuits of the same matroid if and only if $\mathscr{C}$ and $\mathscr{D}$ satisfy the orthogonality axioms.

\subsection{Examples}

In this section, we provide some natural examples of general matroids. More primal examples can be found in the existing literature on Higgs's B-matroids, see for example [8], [27], [29], [31], and [39].

\subsubsection{Uniform Matroids}

Let $E$ be any set and $k$ be a non-negative integer. If

$$
\mathscr{I}=\{I \subseteq E:|I| \leq k\},
$$

then $M=(\mathscr{I}, E)$ is a matroid. It will be called a uniform matroid of rank $\mathrm{k}$. If

$$
\mathscr{I}^{\prime}=\{I \subseteq E:|E \backslash I| \geq k\},
$$


then $M^{*}=\left(\mathscr{I}^{\prime}, E\right)$ is a is also a matroid. It will be called a uniform matroid of co-rank $k$. Matroids $M$ and $M^{*}$ are the dual of each other.

\subsubsection{Cycle and Bond Matroids in Graphs}

There are two standard matroids related to a graph $G$ that we state in this subsection. We say that a finite circuit of a graph is the edge sets of a finite cycle of $G$. Let $G=(V, E)$ be a graph and

$$
\mathscr{I}=\{I \subseteq E: I \text { contains no finite circuits of } G\}
$$

Then $(\mathscr{I}, E)$ is a matroid. It will be called finite-cycle matroid of $G$ and denoted by $M_{F C}(G)$. Clearly, $M_{F C}(G)$ is a finitary matroid for any $G$. The other matroid is called the finite-bond matroid of $G$ denoted by $M_{F B}(G)$ whose circuits are the finite bonds of $G$. (A bond is a minimal non-empty cut.)

When the graph $G$ is finite, these two matroids are dual. If $G$ is infinite, the dual of $M_{F C}(G)$ is not $M_{F B}(G)$ but the full bond matroid $M_{B}(G)$. This is the matroid whose circuits are all the bonds of $G$, finite or infinite: these are the minimal edge sets meeting all the spanning trees of $G$ (connected), the bases of $M_{F C}(G)$. Similarly, the dual of $M_{F B}(G)$ is not $M_{F C}(G)$ but a matroid $M_{T C}(G)$ which its circuits can be infinite.

\subsubsection{The Algebraic Cycle Matroid of a Graph}

Another matroid associated to a graph $G$ is its algebraic cycle matroid. In this subsection, we state this matroid. We say that a set is the algebraic cycle of $\mathrm{G}$ if it is the edge set of a (finite) cycle or a double ray of $G$, (a double ray of $G$ is a 2-way infinite path of $G$ ).

Let $G=(V, E)$ be a graph and

$$
\mathscr{I}=\{I \subseteq E: I \text { contains no algebraic circuits of } G\}
$$




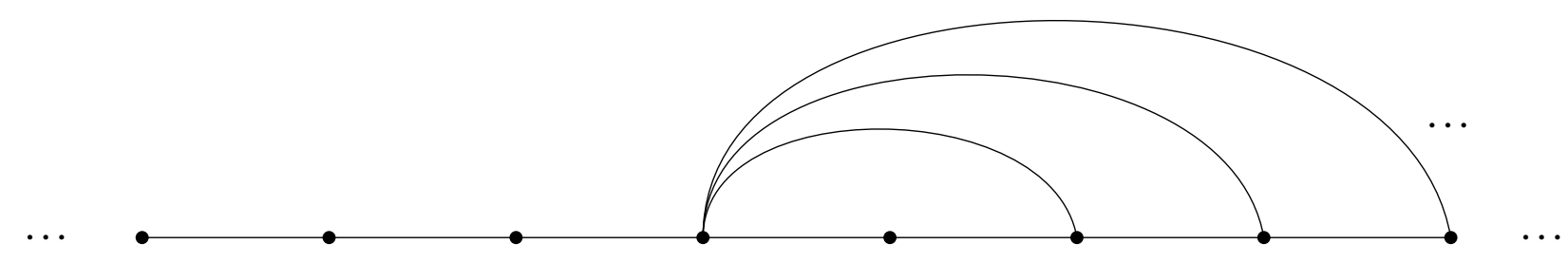

Figure 2.5.1: The Bean graph

Then $(\mathscr{I}, E)$ is not necessary a matroid in every infinite graph. For example [26], the pair $(\mathscr{I}, E)$ is not a matroid when $G$ is the Bean graph shown in Figure 2.5.1.

However, Higgs [26] showed that this is actually the only counterexample. In particular, he proved in [26] that the algebraic cycles of an infinite graph $G$ are the circuits of a matroid on its edge set $E(G)$ if and only if $G$ contains no subdivision of the Bean graph.

\subsubsection{Partition Matroids}

Definition 2.5.1. Let $\left\{E_{i}: i \in I\right\}$ be a partition of the set $E$, and

$$
\mathscr{I}=\left\{I \subseteq E:\left|I \cap E_{i}\right| \leq 1 \text { for each } i \in I\right\} .
$$

Then $M=(E, \mathscr{I})$ is a matroid and is called partition matroid on $E$ corresponding to the partition $E=\bigsqcup_{i \in I} E_{i}$.

Remark. Every bipartite graph induces two partition matroids. Let $G$ be a bipartite graph with sides of vertices $A$ and $B, E=\bigsqcup_{v \in A} E_{v}$ and $E=\bigsqcup_{w \in B} E_{w}$ the partitions of its edge set where each $E_{v}$ is the set of edges incident to the vertex $v$ for $v \in A$ and each $E_{w}$ is the set of edges incident to the vertex $w$ for $w \in B$. We call the partition matroid on $E$ corresponding to the partition $E=\bigsqcup_{v \in A} E_{v}$ the partition matroid determined by side A, and the partition matroid on $E$ corresponding to the partition $E=\bigsqcup_{w \in B} E_{w}$ the partition matroid determined by side $B$.

Let $\left(M_{i} ; i \in I\right)$ be a family of matroids on the same ground set $E$. A packing for this family 


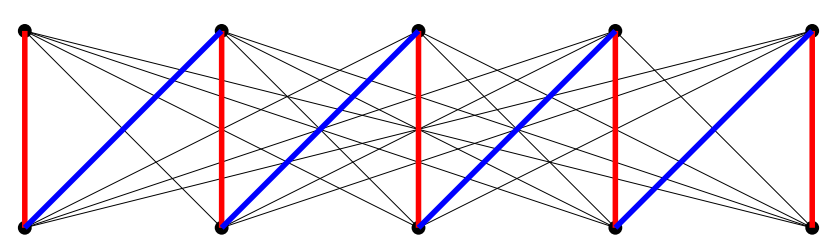

Figure 2.5.2: A packing for the partition matroids determined by sides of a bipartite graph.

of matroids consists of disjoint spanning sets $S_{i}$ for each $M_{i}$. A covering for this family consists of independent sets $I_{i}$ for each $M_{i}$ such that whose union is the set $E$.

Example 2.5.2. Let $G$ be a bipartite graph with sides of vertices $A$ and $B$ and the edge set $E$. Let $M$ and $N$ be the partition matroid determined by sides $A$ and $B$ respectfully. Then any base $B \subseteq E$ of $M$ has exactly one edge incident to a vertex $v$ for any $v \in A$ (Similarly, any base $D \subseteq E$ of $N$ has exactly one edge incident to a vertex $v$ for any $v \in B$ ). For instance, consider the complete bipartite graph $G$ shown in Figure 2.5.2. Let $M$ and $N$ be the partition matroids determined by each side of vertices. Then, the sets of blue and red edges form a packing for the pair of matroids $(M, N)$ and obviously it has no covering.

\subsection{Equicardinality of Bases for Tame Matroids}

If $A$ and $B$ are the sets, then we say that $A$ and $B$ have the same cardinality or that they are equicardinal if and only if there exists a bijection $f: A \rightarrow B$. Higgs [25] proved that assuming the generalized continuum hypothesis, (GCH) any two bases of a general matroid have the same cardinality. Bowler and Geschke [15] show that it is also consistent with ZFC that there is a matroid with bases of two different cardinalities.

The followings are defined in [9]: A matroid $M$ is tame if the intersection of any circuit of $M$ with any co-circuit of $M$ is finite. Otherwise, it is called wild. Note that any finitary and any co-finitary matroid is tame. The existance of a wild matroid is shown in [10].

The following theorem shows [13] that for a tame matroid any two bases have the same cardinality without using any extra axioms beyond ZFC. 
Theorem 2.6.1 ([13]). Let $M$ be a tame matroid and $B$ and $D$ bases of $M$. Then $|B|=|D|$.

Proof. Let $E$ be the ground set of $M$. Suppose, for a contradiction, that $|B| \neq|D|$. Without loss of generality, we can assume that $B \cup D=E$ and $B \cap D=\emptyset$ since otherwise we can replace $M$ by the (tame) matroid

$$
M^{\prime}=\left(M \uparrow_{B \cup D}\right) /(B \cap D)
$$

for which the sets $B \backslash D$ and $D \backslash B$ are bases of different cardinality. Then both $B$ and $D$ are also bases of the dual matroid $M^{*}$.

For each $b \in B$, let $D_{b}$ be the (finite) intersection of the fundamental circuit of $b$ with respect to $D$ and he fundamental co-circuit of $b$ with respect to $D$. Similarly, for each $d \in D$, let $B_{d}$ be the (finite) intersection of the fundamental circuit of $d$ with respect to $B$ and the fundamental co-circuit of $d$ with respect to $B$. Note that for every $d \in D$ and $b \in B$ we have $d \in D_{b}$ if and only if $b \in B_{d}$. Define an equivalence relation $\sim$ on $B$ by $b \sim b^{\prime}$ if and only if

$$
D_{b} \backslash\{b\}=D_{b^{\prime}} \backslash\left\{b^{\prime}\right\} .
$$

Then each equivalence class of $\sim$ is finite. Consider $\mathscr{B}$ to be the set of all equivalence classes of $\sim$ and

$$
\mathscr{D}=\left\{D_{b} \backslash\{b\}: b \in B\right\} .
$$

Then $|\mathscr{B}|=|B|$ and $|\mathscr{D}| \leq|D|$. The function assigning $D_{b} \in \mathscr{D}$ to the equivalence class containing $b$ is an injection so $|\mathscr{B}| \leq|\mathscr{D}|$, which implies that $|B| \leq|D|$. By symmetry, we also have $|D| \leq|B|$ so $|B|=|D|$, which is a contradiction. 


\section{Chapter 3}

\section{Almost Intersection}

\subsection{Introduction}

Suppose we have a family of matroids $\left(M_{k}: k \in K\right)$ on the same ground set $E$. A packing for this family consists of a spanning set $S_{k}$ for each $M_{k}$ such that the $S_{k}$ are all disjoint. The wellknown finite base packing theorem states that if $E$ is finite then the family has a packing if and only if for every subset $Y \subseteq E$ the following holds.

$$
\sum_{k \in K} r_{M_{k} \cdot Y}(Y) \leq|Y| .
$$

This theorem does not extend accurately to finitary matroids [4] (see also [21]). However, it is shown in [6] that the base packing theorem extends to finite families of co-finitary matroids. Bowler and Carmesin show [12] that the base packing theorem extends to arbitrary families of co-finitary matroids.

Similar to packings are coverings: a covering for a family $\left(M_{k}: k \in K\right)$ on the same ground set $E$ consists of an independent set $I_{k}$ for each $M_{k}$ such that the union of all $I_{k}$ covers $E$. And analogously to the base packing theorem, there is a base covering theorem characterizing the finite families of finite matroids admitting a covering.

Bowler and Carmesin [12] proposed this question: "Although not every family of matroids 
has a packing and not every family has a covering, is it always possible to divide the ground set into a part, which has a packing, and a part, which has a covering?"

Definition 3.1.1. A family of matroids $\left(M_{k}: k \in K\right)$ on the same ground set $E$, has the Packing/Covering property if $E$ admits a partition $E=P \sqcup C$ such that $\left(M_{k} \uparrow_{P}: k \in K\right)$ has a packing and $\left(M_{k} . C: k \in K\right)$ has a covering.

Conjecture 3.1.2 (Bowler and Carmesin [12]). Any family of matroids on a common ground set has the Packing/Covering property.

This conjecture is called the Packing/Covering conjecture. Here $M_{k} \uparrow_{p}$ is the restriction of $M_{k}$ to $P$ and $M_{k}$.C is the contraction of $M_{k}$ onto $C$. For finite matroids, the Packing/Covering Conjecture 3.1.2 is true [12]. For infinite matroids, the Packing/Covering Conjecture 3.1.2 and the Matroid Intersection Conjecture are equivalent, and that both are equivalent to Conjecture 3.1.2 for pairs of matroids. Specifically, Bowler and Carmesin proved the followings.

Theorem 3.1.3 (Bowler and Carmesin [12]). $(M, N)$ satisfies the Matroid Intersection Conjecture if and only if $\left(M, N^{*}\right)$ has the Packing/Covering Property.

Corollary 3.1.4 (Bowler and Carmesin [12]). If $M$ and $N$ are matroids on the same ground set, then $M$ and $N$ satisfies the Matroid Intersection Conjecture if and only if $M^{*}$ and $N^{*} d o$.

The Packing/Covering Conjecture 3.1.2 is known to be true for the following cases: Here we say a matroid $M$ on $E$ is nearly finitary if and only if for every $A \subseteq E$ that contains no finite circuits of $M$ there exists a finite $F \subseteq A$ such that $A \backslash F$ is independent in $M$.

- a family of co-finitary matroids.

- a finite family of finitary matroids.

- a finite family of nearly finitary matroids.

- a family of finitary matroids on a countable ground set. 
For the rest of this section assume that $M$ and $N$ are matroids on a common ground set $E$.

Definition 3.1.5. We say that the pair $(M, N)$ has the Almost Intersection Property when there exist almost disjoint $I, J \subseteq E$ such that $\operatorname{cl}_{M}(I) \cup \operatorname{cl}_{N}(J)$ is almost equal to $E$ and $I \cup J$ is almost independent in both $M$ and $N$. We mean here that the sets $I \cap J, E \backslash\left(\operatorname{cl}_{M}(I) \cup \mathrm{cl}_{N}(J)\right)$ and $(I \cup J) \backslash K$ are all finite for some $K \subseteq E$ that is independent in both $M$ and $N$.

Definition 3.1.6. We say that $(S, T)$ is an almost packing of $(M, N)$ if and only if $S$ and $T$ are spanning in $M$ and $N$, respectively, and $S \cap T$ is finite. Analogously, we say that $(I, J)$ is an almost covering of $(M, N)$ when $I$ and $J$ are independent in $M$ and $N$, respectively and $E \backslash(I \cup J)$ is finite. If there exists a partition $E=P \sqcup Q$ of $E$ such that $(M \backslash Q, N \backslash Q)$ has an almost packing and $(M / P, N / P)$ has an almost covering, then we say that $(M, N)$ has the Almost Packing/Covering Property.

Definition 3.1.7. If $F \subseteq E$, then say that $(M, N)$ has the Packing/Covering Property modulo $F$ if and only if there exists a partition $E \backslash F=P \sqcup C$ such that $(M, N) / F \backslash C$ has a packing and $(M, N) \backslash F / P$ has a covering.

The following proposition will be proved in Section 2 .

Proposition 3.1.8. $(M, N)$ has the Almost Intersection Property if and only if $\left(M, N^{*}\right)$ has the Packing/Covering Property modulo a finite set.

The main result of this chapter is the following theorem and will be proved in Section 3.

Theorem 3.1.9. If $(M, N)$ has the Almost Intersection Property, then it satisfies the Matroid Intersection Conjecture.

Note that Theorem 3.1.9 immediately implies the Edmonds' Intersection Theorem (the finite case of the Matroid Intersection Conjecture).

Theorem 3.1.9 follows from Theorem 3.1.3, Proposition 3.1.8 and the following result.

Theorem 3.1.10. The following are equivalent. 
1. $(M, N)$ has the Packing/Covering Property.

2. $(M, N)$ has the Almost Packing/Covering Property.

3. $(M, N)$ has the Packing/Covering Property modulo a finite subset of $E$

The following corollary follows.

Corollary 3.1.11. If $(M, N)$ has the Packing/Covering Property and $A, B \subseteq E$ are finite, then $(M, N) / A \backslash B$ also has the Packing/Covering Property.

Using this new direction and our results we prove the following results in Section 4.

Theorem 3.1.12. If $M$ has finite rank and $N$ is arbitrary, then $(M, N)$ satisfies the Matroid Intersection Conjecture.

Theorem 3.1.13. If $M$ and $N$ are nearly finitary, then $\left(M, N^{*}\right)$ satisfies the Matroid Intersection Conjecture.

For the definition of patchwork matroid see 3.4.3.

Theorem 3.1.14. If $M$ is patchwork and $N$ is arbitrary, then $\left(M, N^{*}\right)$ satisfies the Matroid Intersection Conjecture.

\subsection{Proof of Proposition 3.1.8}

We follow the notation and terminology of [32] and [19].

Let $M$ and $N$ be matroids on the same ground set $E$. A packing for $(M, N)$ is a pair $(S, T)$ of disjoint subsets of $E$ such that $\mathrm{cl}_{M}(S) \cup \mathrm{cl}_{N}(T)=E$. A covering for $(M, N)$ is a pair $(A, B)$ of subsets of $E$ that are independent in $M, N$, respectively, and $A \cup B=E$.

Proof of Proposition 3.1.8. Assume first that there exists a partition $E=P \sqcup Q \sqcup F$ such that $F$ is finite, $\left(M, N^{*}\right) / F \backslash Q$ has a packing and $\left(M, N^{*}\right) \backslash F / P$ has a covering. Then $P$ and $Q$ can be partitioned as $P=S \sqcup T$ and $Q=A \sqcup B$ with $T \subseteq \operatorname{cl}_{M}(S \cup F), S \subseteq \mathrm{cl}_{N^{*}}(T \cup F), A \subseteq \mathrm{cl}_{N}(B \cup F)$ 
and $B \subseteq \mathrm{cl}_{M^{*}}(A \cup F)$. Moreover, we can assume without loss of generality that $S$ is independent in $M$ and $B$ is independent in $N$ (see Figure 3.2.1).

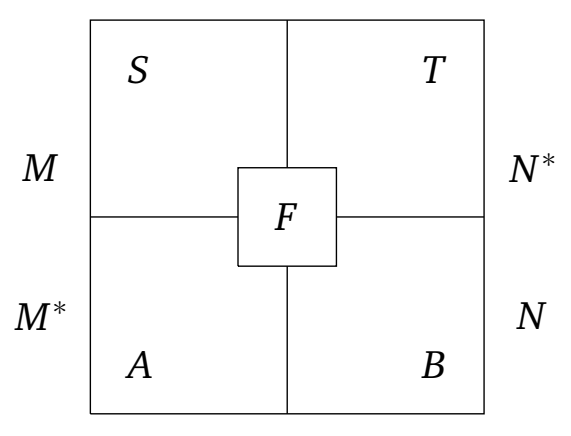

Figure 3.2.1: The sets $F, S, T, A$ and $B$.

Since $T \cup F$ is spanning in $N^{*} \backslash Q$, it follows that $S$ is independent $N / Q$ implying that $S \cup B$ is independent in $N$. Similarly $S \cup B$ is independent in $M$. Let $I=S \cup F$ and $J=B \cup F$. Then $I, J$ are almost disjoint and $I \cup J$ is almost independent in both $M$ and $N$. Moreover, $\operatorname{cl}_{M}(I) \cup \mathrm{cl}_{N}(J)=E$. It follows that $(M, N)$ has the Almost Intersection Property.

Now assume that $(M, N)$ has the Almost Intersection Property. Let $I, J \subseteq E$ be almost disjoint and such that $\operatorname{cl}_{M}(I) \cup \mathrm{cl}_{N}(J)$ is almost equal to $E$ and $I \cup J$ is almost independent in both $M$ and $N$. Without loss of generality, we can assume that $I$ is independent in $M$ and $J$ is independent in $N$. Let $I^{\prime} \subseteq I \backslash J$ and $J^{\prime} \subseteq J \backslash I$ be such that $I \cup J^{\prime}$ is a basis of $M \uparrow_{I \cup J}$ and $J \cup I^{\prime}$ is a basis of $N \uparrow_{I \cup J}$. Note that $(I \cup J) \backslash\left(I^{\prime} \cup J^{\prime}\right)$ is finite. Let $P^{\prime}, Q^{\prime} \subseteq E \backslash(I \cup J)$ be disjoint and such that $E \backslash\left(I \cup J \cup P^{\prime} \cup Q^{\prime}\right)$ is finite with $P^{\prime} \subseteq \operatorname{cl}_{M}(I)$ and $Q^{\prime} \subseteq \operatorname{cl}_{N}(J)$. Let $P=I^{\prime} \cup P^{\prime}$, $Q=J^{\prime} \cup Q^{\prime}$ and $F=E \backslash(P \cup Q)$ (see Figure 3.2.2).

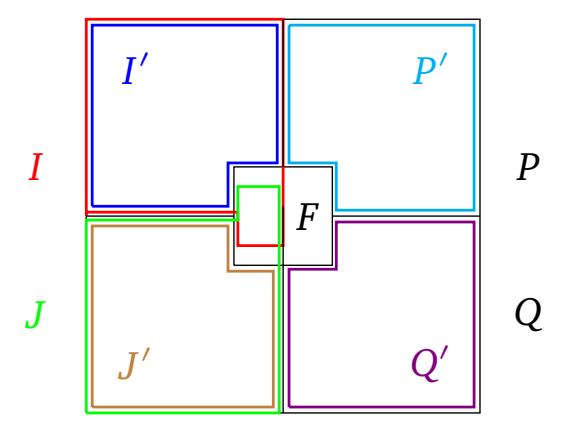

Figure 3.2.2: A packing for $\left(M, N^{*}\right) / F \backslash Q$ and a covering for $\left(M, N^{*}\right) \backslash F / P$. 
Note that $F$ is finite and $P \subseteq \operatorname{cl}_{M}\left(I^{\prime} \cup F\right)$. Moreover, since $I^{\prime} \cup J$ is independent in $N$ and $J$ spans every element of $Q$, it follows that $I^{\prime}$ is independent in $N / Q$ and hence $P^{\prime} \cup F$ is spanning in $(N / Q)^{*}=N^{*} \backslash Q$. It follows that $\left(I^{\prime}, P^{\prime}\right)$ is a packing for $\left(M, N^{*}\right) / F \backslash Q$. Similarly, $\left(J^{\prime}, Q^{\prime}\right)$ is a packing for $\left(N, M^{*}\right) / F \backslash P$ and hence it is a covering for $\left(M, N^{*}\right) \backslash F / P$.

\subsection{Proof of Theorems 3.1.9 and 3.1.10}

Throughout this section we assume that $M$ and $N$ are matroids on a common ground set $E$.

A semi-packing for $(M, N)$ is a pair $(B, D)$ of subsets of $E$ that are spanning in $M$ and $N$, respectively, with a minimal possible intersection. That is such that if $\left(B^{\prime}, D^{\prime}\right)$ is another pair of subsets of $E$ that are spanning in $M$ and $N$, respectively, and $B^{\prime} \cap D^{\prime} \subseteq B \cap D$ then $B^{\prime} \cap D^{\prime}=$ $B \cap D$. A semi-covering for $(M, N)$ is defined analogously as a pair of independent subsets with a maximal possible union. Note that if $(M, N)$ has an almost packing, then it has a semi-packing and if it has an almost covering, then it has a semi-covering.

Let $B$ and $D$ be independent in $M$ and $N$, respectively. A $(B, D)$-exchange $M$-chain is a finite sequence $\left(e_{1}, e_{2}, \ldots, e_{n}\right)$ of elements of $E$ such that for each $i \in\{1,2, \ldots, n-1\}$ the elements $e_{i}$ and $e_{i+1}$ are distinct and:

- if $i$ is odd, then there exists a circuit $C$ of $M$ with $e_{i}, e_{i+1} \in C \subseteq B \cup\left\{e_{i}\right\}$;

- if $i$ is even, then there exists a circuit $C$ of $N$ with $e_{i}, e_{i+1} \in C \subseteq D \cup\left\{e_{i}\right\}$.

We say that such a chain is from $e_{1}$ to $e_{n}$.

A $(B, D)$-exchange $N$-chain is defined analogously with the words "even" and "odd" interchanged. A $(B, D)$-exchange chain refers to either of these notions.

The following lemmas are proved in [6].

Lemma 3.3.1. If there exists an $\left(I_{1}, I_{2}\right)$-exchange chain from $y$ to $x$ with $y \notin I_{1} \cup I_{2}$, then there exists an $\left(I_{1}^{\prime}, I_{2}^{\prime}\right)$-exchange chain from $y$ to $x$ such that $y \in I_{1} \cup I_{2}$. 
Remark. In the proof of Lemma 3.3.1 chains are used in order to alter the sets $I_{1}$ and $I_{2}$; the change is in a single element. Nevertheless, to accomplish this change, exchange chain of arbitrary length may be required; for instance, a chain of length four is needed to handle the configuration depicted in Figure 3.3.1.

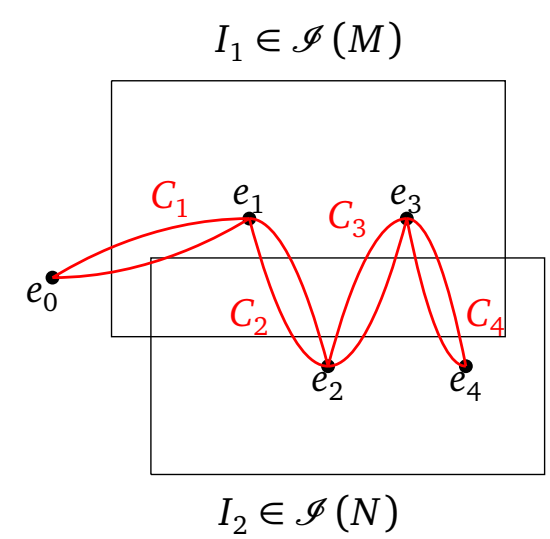

(a) the initial representation

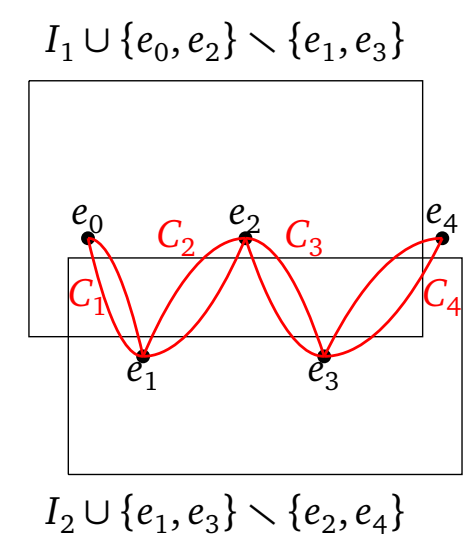

(b) the obtained representation

Figure 3.3.1: An even exchange chain of length 4.

Lemma 3.3.2. Let $B$ and $D$ be independent in $M$ and $N$, respectively. If there exists $a(B, D)-$ exchange $M$-chain from $d \in E \backslash(B \cup D)$ to $e \in B \cap D$, then there exist $B^{\prime}$ and $D^{\prime}$ that are independent in $M$ and $N$, respectively, such that $B^{\prime} \cap D^{\prime}=(B \cap D) \backslash\{e\}, \operatorname{cl}_{M}(B) \subseteq \operatorname{cl}_{M}\left(B^{\prime}\right)$ and $\mathrm{cl}_{N}(D) \subseteq \mathrm{cl}_{N}\left(D^{\prime}\right)$.

The following lemma is the key technical result that will be used in the proof of the main result.

Lemma 3.3.3. If $(M, N)$ has a semi-packing, then it has the Packing/Covering Property.

Proof. Let $(B, D)$ be a semi-packing for $(M, N)$. Without loss of generality, we can assume that $B$ and $D$ are bases of $M$ and $N$, respectively. Let $B^{\prime}$ be the set of all $e \in B$ to which there exists a $(B, D)$-exchange chain from an element of $E \backslash(B \cup D)$. Similarly, let $D^{\prime}$ consist of those $e \in D$ to which there exists a $(B, D)$-exchange chain from $E \backslash(B \cup D)$ (see Figure 3.3.2). 


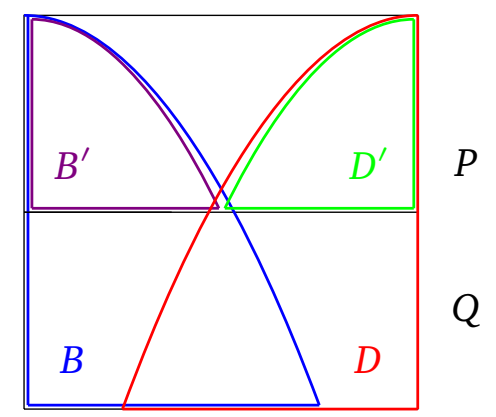

Figure 3.3.2: The sets $B, D, B^{\prime}$ and $D^{\prime}$.

Note that $B^{\prime} \cap D=\varnothing$ and $D^{\prime} \cap B=\varnothing$, since if $e \in B \cap D$ and there is a $(B, D)$-exchange $M$-chain from $d \in E \backslash(B \cup D)$ to $e$, then Lemma 3.3.2 implies that there exist $B^{\prime \prime}$ and $D^{\prime \prime}$ that are bases of $M$ and $N$, respectively, such that

$$
B^{\prime \prime} \cap D^{\prime \prime}=(B \cap D) \backslash\{e\} .
$$

Since $B^{\prime \prime} \cap D^{\prime \prime}$ is a proper subset of $B \cap D$ and $(B, D)$ is a semi-packing we get a contradiction. Similarly, the existence of a $(B, D)$-exchange $N$-chain would lead to a contradiction.

Let

$$
P=B^{\prime} \cup D^{\prime} \cup(E \backslash B \cup D),
$$

(see Figure 3.3.2). We claim that $\left(B^{\prime}, D^{\prime}\right)$ is a packing for $\left(M \uparrow_{P}, N \uparrow_{P}\right)$. If $e \in E \backslash(B \cup D)$, then the definition of exchange chains implies that $e$ is spanned by $B^{\prime}$ in $M$ and by $D^{\prime}$ in $N$. If $e \in B^{\prime}$, then $e$ is spanned by $D$ in $N$ so there exists a circuit $C$ of $N$ with $\{e\} \in C \subseteq D \cup\{e\}$. Since $e \in B^{\prime}$, it follows that there exists a $(B, D)$-exchange chain from $E \backslash(B \cup D)$ to each element of $C \backslash\{e\}$ implying that $C \backslash\{e\} \subseteq D^{\prime}$. Thus $e$ is spanned by $D^{\prime}$ in $N$. Similarly, each element of $D^{\prime}$ is spanned by $B^{\prime}$ in $M$ completing the proof that $\left(B^{\prime}, D^{\prime}\right)$ is a packing for $M \uparrow_{P}$.

Let $Q=E \backslash P$ (see Figure 3.3.2). $\hat{B}=B \cap Q$ and $\hat{D}=D \cap Q$. We claim that $(\hat{B}, \hat{D})$ is a covering of $(M . Q, N . Q)$. Clearly $\hat{B} \cup \hat{D}=Q$. Since $B=B^{\prime} \cup \hat{B}$ is independent in $M$ and $B^{\prime}$ spans $P$ in $M$, it follows that $\hat{B}$ is independent in M.Q. Similarly, $\hat{D}$ is independent in N.Q completing the proof of the lemma. 
Since $(M, N)$ has the Packing/Covering Property if and only if $\left(M^{*}, N^{*}\right)$ does so, the following corollary follows.

Corollary 3.3.4. If $(M, N)$ has a semi-covering, then it has the Packing/Covering Property.

The proof of the following lemma is routine.

Lemma 3.3.5. Let I be independent in $M$ and $F \subseteq E$ be finite. Then there exists $I^{\prime} \subseteq I$ that is independent in $M / F$ with $I \backslash I^{\prime}$ finite.

The following corollary follows.

Corollary 3.3.6. If $(M, N)$ has an almost covering and $F \subseteq E$ is finite, then $(M / F, N / F)$ has an almost covering.

Now we are ready to prove Theorem 3.1.10.

Proof of Theorem 3.1.10. It is clear that 1. implies 2., which implies 3. It suffices to show that 3. implies 1.

Assume that $(M, N)$ has the Packing/Covering Property modulo finite $F \subseteq E$. Let $P \sqcup Q$ be a partition of $E \backslash F$ such that $(M, N) / F \backslash Q$ has a packing and $(M, N) \backslash F / P$ has a covering. Since $F$ is finite, it follows that $(M \backslash Q, N \backslash Q)$ has an almost packing and hence it has a semi-packing. Consequently, Lemma 3.3.3 implies that $(M \backslash Q, N \backslash Q)$ has the Packing/Covering Property. Let $E \backslash Q=P^{\prime} \sqcup Q^{\prime}$ be a partition of $E \backslash Q$ such that $\left(M \uparrow_{P^{\prime}}, N \uparrow_{P^{\prime}}\right)$ has a packing $(S, T)$ and $(M, N) \backslash Q / P^{\prime}$ has a covering $(A, B)$ (see Figure 3.3.3).

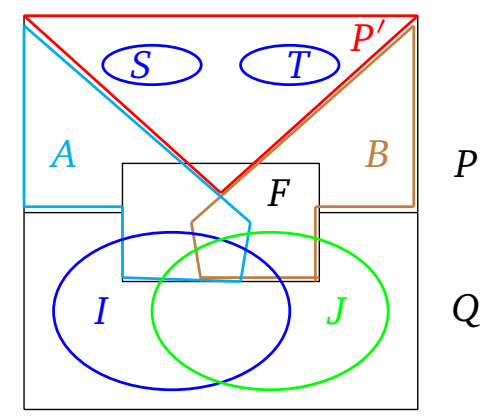

Figure 3.3.3: A packing $(S, T)$ for $\left(M \uparrow_{P^{\prime}}, N \uparrow_{P^{\prime}}\right)$ and a covering $(A, B)$ for $(M, N) \backslash Q / P^{\prime}$. 
A covering of $(M, N) \backslash F / P$ is an almost covering of $(M / P, N / P)$ and $P^{\prime} \backslash P$ is finite so Corollary 3.3.6 implies that $\left(M /\left(P \cup P^{\prime}\right), N /\left(P \cup P^{\prime}\right)\right)$ has an almost covering $(I, J)$. Since $F$ is finite, it follows that $((A \cup I) \backslash F,(B \cup J) \backslash F)$ is an almost covering of $\left(M / P^{\prime}, N / P^{\prime}\right)$. Since $\left(M / P^{\prime}, N / P^{\prime}\right)$ has a semi-covering, Corollary 3.3.4 implies that it has the Packing/Covering Property.

Let $P^{\prime \prime} \sqcup Q^{\prime \prime}$ be a partition of $E \backslash P^{\prime}$ such that $(M, N) / P^{\prime} \backslash Q^{\prime \prime}$ has a packing $\left(S^{\prime}, T^{\prime}\right)$ and $(M, N) / P^{\prime} / P^{\prime \prime}$ has a covering $\left(A^{\prime}, B^{\prime}\right)$ (see Figure 3.3.4).

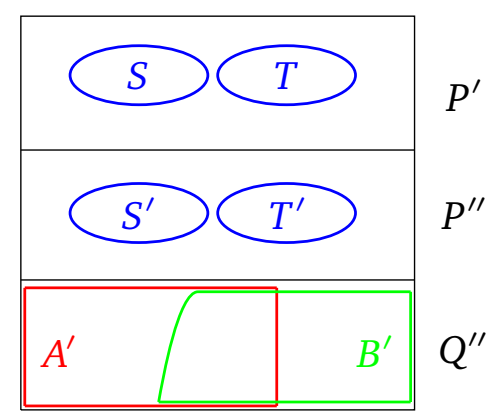

Figure 3.3.4: A packing $\left(S^{\prime}, T^{\prime}\right)$ for $(M, N) / P^{\prime} \backslash Q^{\prime \prime}$ and a covering $\left(A^{\prime}, B^{\prime}\right)$ for $(M, N) / P^{\prime} / P^{\prime \prime}$.

Then $\left(S \cup S^{\prime}, T \cup T^{\prime}\right)$ is a packing for $\left(M \backslash Q^{\prime \prime}, N \backslash Q^{\prime \prime}\right)$ implying that $(M, N)$ has the Packing/Covering Property.

\subsection{Proof of Theorems 3.1.12, 3.1.13, and 3.1.14}

Proof of Theorem 3.1.12. Let $M$ be a matroid of finite rank, and $N$ be an arbitrary matroid. Observe that $\left(M^{*}, N\right)$ has an almost covering. Hence $\left(M^{*}, N\right)$ has a semi-covering and Corollary 3.3.4 implies that $\left(M^{*}, N\right)$ has the Packing/Covering Property. By Theorem 3.1.3 the pair $(M, N)$ satisfies the Matroid Intersection Conjecture.

A matroid $M$ on $E$ is nearly finitary if and only if for every $A \subseteq E$ that contains no finite circuits of $M$ there exists a finite $F \subseteq A$ such that $A \backslash F$ is independent in $M$. Assume that $M$ and $N$ are matroids on the same ground set $E$. Let $M \vee N$ be the set system $M \vee N=(E, \mathscr{I}(M \vee N))$, 
where

$$
\mathscr{I}(M \vee N)=\{I \cup J: I \in \mathscr{I}(M), J \in \mathscr{I}(N)\} .
$$

The following result is proved in [6].

Theorem 3.4.1. If $M$ and $N$ are nearly finitary, then $M \vee N$ is a nearly finitary matroid.

In [7] it is proved that if $M \vee N^{*}$ is a matroid, then $(M, N)$ satisfies the Intersection Conjecture. In particular, the following result holds. We can use Corollary 3.3.4 to provide an alternative proof.

Theorem 3.4.2. If $M$ and $N$ are nearly finitary, then $(M, N)$ has the Packing/Covering Property.

Proof. By Theorem 3.4.1, $M \vee N$ is a matroid. If $I$ and $J$ are independent in $M$ and $N$, respectively, with $I \cup J$ being a basis of $M \vee N$, then $(I, J)$ is a semi-covering of $(M, N)$. By Corollary 3.3.4, $(M, N)$ has the Packing/Covering Property.

Proof of Theorem 3.1.13. By Theorem 3.4.2 and Theorem 3.1.3 it follows.

In [16] patchwork matroids are introduced and proved to satisfy the following characterization. Here $K \triangle B=(K \backslash B) \cup(B \backslash K)$.

Theorem 3.4.3. The matroid $M$ is patchwork if and only if for every $K \subseteq E$ one of the following conditions holds:

1. $K$ is independent in $M$.

2. $K$ is spanning in $M$.

3. There exists a basis $B$ of $M$ with finite $K \triangle B$.

Lemma 3.3.3 implies the following result.

Theorem 3.4.4. If $M$ is patchwork and $N$ is arbitrary, then $(M, N)$ has the Packing/Covering Property. 
Proof. Let $D$ be a basis of $N$ and $K=E \backslash D$. If $K$ is independent in $M$, then $(M, N)$ has a covering. If $K$ is spanning in $M$, then $(M, N)$ has a packing. Otherwise, by Theorem 3.4.3, there exists a basis $B$ of $M$ with finite $K \triangle B$. Then $(B, D)$ is an almost packing for $(M, N)$. Hence $(M, N)$ has a semi-packing and Lemma 3.3.3 implies that $(M, N)$ has the Packing/Covering Property.

Proof of Theorem 3.1.14. By Theorem 3.4.4 and Theorem 3.1.3 it follows. 


\section{Chapter 4}

\section{Critical Sets}

\subsection{Introduction}

One of the main cases in which the Matroid Intersection Conjecture is proved to be true is the case of a pair of partition matroids.

Theorem 4.1.1. The Matroid Intersection Conjecture is true when $M$ and $N$ are partition matroids on set $E$.

This result follows from Theorem 4.1.2, as we will show in Section 2. Theorem 4.1.2 was conjectured by Erdős (see for example [1]). Use [28] for terminology and notations not defined here.

Theorem 4.1.2 (Aharoni [2]). Let $G=(M, W, E)$ be a bipartite graph. Then there exists a matching $f$ and a cover $C$ of $G$ such that

1. every vertex in $C$ is an endpoint of an edge of $f$.

2. no edge of $f$ has both endpoints in $C$.

This implication inspired us to work on the the Matroid Intersection Conjecture in a new direction. The countable case of Theorem 4.1.2 was proved by Podewski and Steffens in [33]. 
The uncountable case was proved by Aharoni [2] in which its fundamental step was proved by Aharoni, Nash-Williams, and Shelah in [3]. The main purpose of Chapter 4 is to generalize the techniques used in that development (described in [28]) beyond partition matroids.

In this chapter we will introduce some new concepts and techniques in matroid theory and develop some new results. In chapter 5 we will use these techniques to prove the Matroid Intersection Conjecture is true for a particular family of matroids. We are convinced that the results in this chapter can be used to prove the Matroid Intersection Conjecture in more general cases as well.

Followings are the results (described in [28]) that motivated us to define and develop the concepts, techniques, and results in this chapter.

Let $\mathscr{F}=\left(F_{i}: i \in I\right)$ be a family of sets. A choice function for $\mathscr{F}$ is a function $f: I \rightarrow \mathscr{F}(I)$ such that $f(i) \in F_{i}$ for each $i \in I$. We say that a family $\mathscr{F}$ is matchable if and only if there is an injective choice function $f: I \rightarrow \mathscr{F}(I)$.

Theorem 4.1.3 (P. Hall [24]). Let $\mathscr{F}=\left(F_{i}: i \in I\right)$ be a finite family of finite sets. Then $\mathscr{F}$ is matchable if and only if

(XO) for every finite $J \subseteq I$ we have $|\mathscr{F}(J)| \geq|J|$.

The condition (X0) is sufficient for a more general family of sets to be matchable.

Theorem 4.1.4 (Brualdi [17]). Let $\mathscr{F}=\left(F_{i}: i \in I\right)$ be a family of finite sets. Then $\mathscr{F}$ is matchable if and only if

(XO) for every finite $J \subseteq I$ we have $|\mathscr{F}(J)| \geq|J|$.

The condition (X0) is not sufficient for a family of arbitrary sets to be matchable (see for example [28]). To find a sufficient condition for a family of sets $\mathscr{F}=\left(F_{i}: i \in I\right)$ to be matchable the following is introduced (see [33] and [37]).

Definition 4.1.5. Let $\mathscr{F}=\left(F_{i}: i \in I\right)$ be a family of sets. A subset $K \subseteq I$ is called critical for $\mathscr{F}$ if and only if 
1. $\left.\mathscr{F}\right|_{K}=\left\{F_{i}: i \in K\right\}$ is matchable, and

2. the range of any matching for $\mathscr{F} \uparrow_{K}$ is $\mathscr{F}(K)=\bigcup_{i \in K} F_{i}$.

Observe that if $\mathscr{F}=\left(F_{i}: i \in I\right)$ is matchable, then we have the following:

(X1) there is no critical set $K \subseteq I$ and $i \in I \backslash K$ with $F_{i} \subseteq \mathscr{F}(K)$.

It is shown in [33] that a countable family is matchable if and only if condition (X1) holds.

Theorem 4.1.6 ([33]). Let $\mathscr{F}=\left(F_{i}: i \in I\right)$ be a family of sets and I countable. Then $\mathscr{F}$ is matchable if and only if

(X1) there is no critical set $K \subseteq I$ and $i \in I \backslash K$ with $F_{i} \subseteq \mathscr{F}(K)$.

Theorem 4.1.6 implies the following [33].

Theorem 4.1.7 ([33]). Let $G=(M, W, E)$ be a bipartite graph with $M$ countable and $\mathscr{F}=$ $\left\{F_{i}: i \in M\right\}$ with $F_{i}=\{w \in W ;(i, w) \in E\}$. The followings are equivalent:

1. $M$ is matchable.

2. (X1) there is no critical set $K \subseteq M$ for $\mathscr{F}$ and $i \in M \backslash K$ such that $F_{i} \subseteq \mathscr{F}(K)$.

Theorem 4.1.7 is equivalent to the following (see for example [28]).

Theorem 4.1.8. Let $G=(M, W, E)$ be a bipartite graph with $M$ countable. Then there exist a matching $f$ and a cover $C$ of $G$ such that

1. every vertex in $C$ is an endpoint of an edge of $f$.

2. No edge of $f$ has both endpoints in $C$.

Finally, Theorem 4.1.8 implies the Matroid Intersection Conjecture is true for partition matroids on a countable set.

This chapter is organized as follows: In Section 2, we show the proof of Theorem 4.1.1. In Sections 3, we introduce critical sets for matroids and we show their connection with critical 
sets for family of sets. In section 4 , we prove the main results of this chapter which are the followings:

Here we say $a \in E$ is essential for $(M, N)$ if and only if $(M, N)$ has a covering and $(M, N) /\{a\}$ has no covering. We say $A \subseteq E$ is critical for $(M, N)$ if and only if $\left(M \uparrow_{A}, N \uparrow_{A}\right)$ has a covering and each covering $(I, J)$ for $\left(M \uparrow_{A}, N \uparrow_{A}\right)$ is also a packing. For the definition of special covering see Definition 4.4.2.

Theorem 4.1.9. Let $(I, J)$ be a special covering and $a \in E$ essential for $(M, N)$. Then there is a critical set $A \subseteq E$ for $(M, N)$ such that $a \in A$.

Theorem 4.1.10. If $(M, N)$ has a covering, then there exists a maximal critical set.

Corollary 4.1.11. Let $(I, J)$ be a special covering and $E^{\prime} \subseteq E$ be such that every $a \in E^{\prime}$ is essential for $(M, N)$. Then there exist a critical set $K \subseteq E$ such that $E^{\prime} \subseteq K$.

\subsection{Proof of Theorem 4.1.1}

In the following, we show that Theorem 4.1.2 implies the Matroid Intersection Conjecture is true for a pair of partition matroids.

Proof. Let $M$ be a partition matroid corresponding to partition $E=\bigsqcup_{i \in I} E_{i}$ and $N$ a partition matroid corresponding to $E=\bigsqcup_{j \in J} E_{j}^{\prime}$. We want to define a bipartite graph $G$ with sides of vertices to be the sets $I$ and $J$ and the sets of edges to be the set $E$. Let $e \in E$ be arbitrary, $i \in I$ such that $e \in E_{i}$ and $j \in J$ such that $e \in E_{j}^{\prime}$. Then in the graph $G$, we let $e$ to be an edge with endpoints $i$ and $j$. By the theorem 4.1.2, there is a matching $f$ and a cover $C$ of $G$ such that

1. every vertex in $C$ is an endpoint of an edge of $f$.

2. No edge of $f$ has both endpoints in $C$.

Observe that matroid $M$ is the partition matroid determined by side $I$ and matroid $N$ is the partition matroid determined by side $B$ in the graph $G$. Observe that the set of edges in matching $f$ is an independent set in both matroids $M$ and $N$. Let $A \subseteq f$ be such that every edge in $A$ 
has an end point in $C \cap I$ and $B \subseteq f$ be such that every edge in $B$ has an endpoint in $C \cap J$. It remain to show that $\operatorname{cl}_{M}(A) \cup \mathrm{cl}_{N}(B)=E$. It is clear that $\mathrm{cl}_{M}(A) \cup \mathrm{cl}_{N}(B) \subseteq E$. So, it is enough to show that $E \subseteq \operatorname{cl}_{M}(A) \cup \mathrm{cl}_{N}(B)$. Let $e \in E$ be arbitrary. If $e \in A$, we have clearly $e \in \mathrm{cl}_{M}(A)$ and if $e \in B$, we have $e \in \operatorname{cl}_{N}(B)$. If $e \notin f$, then $e$ has an endpoint in either $A$ or $B$. If $e$ has an endpoint $v$ in $A$, then there is an edge in $A$ with the same endpoint $v$. Thus $e \in \operatorname{cl}_{M}(A)$. Similarly, if $e$ has an endpoint in $B$, it can be proved that $e \in \mathrm{cl}_{N}(B)$. This implies that $E \subseteq \mathrm{cl}_{M}(A) \cup \mathrm{cl}_{N}(B)$.

\subsection{Critical Sets for Matroids}

In this section we first introduce critical sets for matroids and then we show the equivalency between critical sets for a family of sets and critical sets for matroids. Throughout this section we assume that $M$ and $N$ are matroids on a common ground set $E$.

Definition 4.3.1. We say $A \subseteq E$ is critical for $(M, N)$ if and only if $\left(M \uparrow_{A}, N \uparrow_{A}\right)$ has a covering and each covering $(I, J)$ for $\left(M \uparrow_{A}, N \uparrow_{A}\right)$ is also a packing.

Theorem 4.3.2. Let $G=(I, J, E)$ be a bipartite graph and $\mathscr{F}=\left(F_{i}: i \in I\right)$ a family of sets with $F_{i}=\{j \in J:(i, j) \in E\}$ for every $i \in I$. Let $(M, N)$ be the partition matroids determined by sides $I$ and $J$ respectfully,

$$
\mathscr{K}=\{K \subseteq I: K \text { is a critical set for the family } \mathscr{F}\}
$$

and

$$
\mathscr{A}=\left\{E^{\prime} \subseteq E: E^{\prime} \text { is a critical set for the matroids }\left(M^{*}, N\right)\right\} \text {. }
$$

Then there exists a bijection between $\mathscr{K}$ and $\mathscr{A}$.

Proof. Let $K \in \mathscr{K}$. Let $G^{\prime}$ be the sub-graph of $G$ induced by restriction to the vertices $(K, \mathscr{F}(K))$ and $E^{\prime}$ the set of edges of $G^{\prime}$. We want show that $E^{\prime}$ is a critical set for $\left(M^{*}, N\right)$. Since $K$ is critical for $\mathscr{F}, K$ is matchable. Let $f$ be a matching for $K$. Then $\left(E^{\prime} \backslash f, f\right)$ is a covering for 
$\left(M^{*} \uparrow_{E^{\prime}}, N \uparrow_{E^{\prime}}\right)$. To show that every covering for $\left(M^{*} \uparrow_{E^{\prime}}, N \uparrow_{E^{\prime}}\right)$ is a also a packing, it is enough to show that for every covering $(A, B)$ for $\left(\left.M^{*}\right|_{E^{\prime}}, N \uparrow_{E^{\prime}}\right)$ we have $A \cap B=\emptyset$. Suppose, for a contradiction, that there exists a covering $(A, B)$ for $\left(M^{*} \uparrow_{E^{\prime}}, N \uparrow_{E^{\prime}}\right)$ such that $A \cap B \neq \emptyset$. Let $e \in A \cap B$ and $e=\left(i^{\prime}, j^{\prime}\right)$ for some $i^{\prime} \in I$ and $j^{\prime} \in J$. Let

$$
H_{i}=\left\{j \in F_{i}:(i, j) \in A\right\}
$$

for every $i \in K$. Since $A$ is independent in $M^{*}$, for every vertex $i \in K$, there is $j \in \mathscr{F}(K)$ such that $e_{i}=(i, j) \notin A$ and so $\left\{j \in F_{i} \backslash H_{i}\right\} \neq \emptyset$. Let

$$
\left\{\begin{array}{c}
f: K \rightarrow \mathscr{F}(K) \\
f(i)=\left\{j \in F_{i} \backslash H_{i}\right\}
\end{array} .\right.
$$

We show that for any two $i_{1}, i_{2} \in K$, we have $f\left(i_{1}\right) \cap f\left(i_{2}\right)=\emptyset$ which implies that there exists an injective choice function for $K$. Suppose, for a contradiction, that there are $i_{1}, i_{2} \in K$ with $j \in f\left(i_{1}\right) \cap f\left(i_{2}\right)$. This means $e_{i_{1}}=\left(i_{1}, j\right) \notin A$ and $e_{i_{2}}=\left(i_{2}, j\right) \notin A$. So, $\left\{e_{i_{1}}, e_{i_{2}}\right\} \subseteq B$. But $\left\{e_{i_{1}}, e_{i_{2}}\right\}$ is a circuit of matroid $N$ which is a contradiction with $B$ is independent in $N$. Thus, there is an injective choice function $f^{\prime} \subseteq f$ which is a matching for $K$ and since $K$ is critical we have $\operatorname{ran}\left(f^{\prime}\right)=\mathscr{F}(K)$. So, $j^{\prime} \in \operatorname{ran}\left(f^{\prime}\right)$, which implies there is $r \in K$ such that $e_{r}=\left(r, j^{\prime}\right) \notin A$. Since $e=\left(i^{\prime}, j^{\prime}\right) \in A, r \neq i^{\prime}$, which implies $e_{r}$ and $e$ are distinct edges. The set $\left\{e_{r}, e\right\} \subseteq B$ is a circuit of $N$ which is a contradiction with $B$ is independent in $N$. This completes the proof that $E^{\prime}$ is a critical set for $\left(M^{*}, N\right)$.

Now let $E^{\prime} \in \mathscr{A}$. Since $E^{\prime}$ is a critical set for matroids $\left(M^{*}, N\right)$, the pair $\left(M^{*} \uparrow_{E^{\prime}}, N \uparrow_{E^{\prime}}\right)$ has a covering. Let $(A, B)$ be a covering for $\left(M^{*} \uparrow_{E^{\prime}}, N \uparrow_{E^{\prime}}\right)$. Consider the sub-graph $G^{\prime}$ obtained by restricting the graph $G$ to the edge sets $E^{\prime}$ and let $\left(V_{1}, V_{2}\right)$ be the sides of vertices of $G^{\prime}$. First observe that since $E^{\prime}$ is a critical set for matroids $\left(M^{*}, N\right), V_{2}=\mathscr{F}\left(V_{1}\right)$. Suppose for a contradiction, that $\mathscr{F}\left(V_{1}\right) \backslash V_{2} \neq \emptyset$. Let $\left(i, j_{1}\right) \in \mathscr{F}\left(V_{1}\right) \backslash V_{2}$. There exists some $j_{2} \in V_{2}$ such that $\left(i, j_{2}\right) \in E^{\prime}$.Let $\left(A^{\prime}, B^{\prime}\right)$ be a covering for $\left(M^{*} \uparrow_{E^{\prime}}, N \uparrow_{E^{\prime}}\right)$ such that $\left(i, j_{2}\right) \in B^{\prime}$. Then $\left(A^{\prime}, B^{\prime}\right)$ is 
not a packing for $\left(\left.M^{*}\right|_{E^{\prime}}, N \uparrow_{E^{\prime}}\right)$ because $\left(i, j_{1}\right) \notin E^{\prime}$.This is a contradiction with $E^{\prime}$ is a critical set for $\left(M^{*}, N\right)$. Therefore, $V_{2}=\mathscr{F}\left(V_{1}\right)$. Let $i \in V_{1}$, then there exists some $j \in V_{2}$ such that $(i, j) \notin A$ because $A$ is independent in $M^{*}$. Let

$$
\left\{\begin{array}{c}
g: V_{1} \rightarrow V_{2} \\
g(i)=\left\{j \in V_{2}:(i, j) \notin A\right\}
\end{array} .\right.
$$

We want to show that $V_{1}$ is a critical set for the family $\mathscr{F}$. We first show that for any $i_{1}, i_{2} \in V_{1}$, we have $g\left(i_{1}\right) \cap g\left(i_{2}\right)=\emptyset$. Suppose, for a contradiction, that there are some $i_{1}, i_{2} \in V_{1}$ such that $g\left(i_{1}\right) \cap g\left(i_{2}\right) \neq \emptyset$. Let $j \in g\left(i_{1}\right) \cap g\left(i_{2}\right)$. This implies that $\left(i_{1}, j\right),\left(i_{2}, j\right) \notin A$ and therefore $\left(i_{1}, j\right),\left(i_{2}, j\right) \in B$. But $\left\{\left(i_{1}, j\right),\left(i_{2}, j\right)\right\}$ is a circuit of $N$ and $\left\{\left(i_{1}, j\right),\left(i_{2}, j\right)\right\} \subseteq B$ is a contradiction with $B$ is independent in $N$. So, for any $i_{1}, i_{2} \in V_{1}$, we have $g\left(i_{1}\right) \cap g\left(i_{2}\right)=\emptyset$ and since $g(i) \neq \emptyset$ for any $i \in V_{1}$ this implies that $V_{1}$ is matchable. It remains to show that for any matching $f$ for $V_{1}, \operatorname{ran}(f)=\mathscr{F}\left(V_{1}\right)$. Let $f$ be a matching for $V_{1}$, then $\left(E^{\prime} \backslash f, f\right)$ is a covering for $\left(M^{*} \uparrow_{E^{\prime}}, N \uparrow_{E^{\prime}}\right)$. Since $E^{\prime}$ is a critical set for $\left(M^{*}, N\right)$, every covering for $\left(M^{*} \uparrow_{E^{\prime}}, N \uparrow_{E^{\prime}}\right)$ is also a packing. This implies that $f$ is a base of $N \uparrow_{E^{\prime}}$. This means for every $j \in V_{2}$, there exists some $i$ such that the edge $(i, j) \in f$. This implies $\operatorname{ran}(f)=V_{2}$.

Definition 4.3.3. Let $\mathscr{F}=\left(F_{i}: i \in I\right)$ be a family of sets such that $I \cap \mathscr{F}(I)=\emptyset$. The bipartite graph corresponding to $\mathscr{F}$ is the graph $G_{\mathscr{F}}=(I, \mathscr{F}(I), E)$ where $E=\left\{\{i, a\}: i \in I, a \in F_{i}\right\}$.

Definition 4.3.4. Let $\mathscr{F}=\left(F_{i}: i \in I\right)$ be a family of sets such that $I \cap \mathscr{F}(I)=\emptyset$ and $G_{\mathscr{F}}=$ $(I, \mathscr{F}(I), E)$ the bipartite graph corresponding to $\mathscr{F}$. The corresponding partition matroids to $\mathscr{F}$ is the partition matroids determined by sides $I$ and $J$ respectfully.

Proposition 4.3.5. Let $\mathscr{F}=\left(F_{i}: i \in I\right)$ be a family of sets and $(M, N)$ the corresponding partition matroids to $\mathscr{F}$. Then failure of the condition (X1) for $\mathscr{F}$ induces a unique critical set for the $\left(M^{*}, N\right)$. 
Proof. Let $K \subseteq I$ be a critical set for $\mathscr{F}$ and $i \in I \backslash K$ such that $F_{i} \subseteq \mathscr{F}(K)$. Consider

$$
G=(I, \mathscr{F}(I), E)
$$

the corresponding bipartite graph to the family $\mathscr{F}=\left(F_{i}: i \in I\right)$ and $(M, N)$ the partition matroids determined by the sides $I$ and $\mathscr{F}(I)$ respectfully. Let $E^{\prime}$ be the edge set of $G$ restricted to the sides $(K, \mathscr{F}(K))$ and

$$
E_{i}=\left\{e \in E: e=(i, j) \text { for some } j \in F_{i}\right\}
$$

and $U_{i}=E_{i} \backslash\{e\}$ for some $e \in E_{i}$. We want to show that $E^{\prime \prime}=E^{\prime} \cup U_{i}$ is a critical set for $\left(M^{*}, N\right)$. We first show that $\left(M^{*} \Gamma_{E^{\prime \prime}}, N \uparrow_{E^{\prime \prime}}\right)$ has a covering. Let $f$ be a matching for $K$. Then

$$
\left(\left(E^{\prime} \backslash f\right) \cup U_{i}, f\right)
$$

is a covering for $\left(M^{*} \uparrow_{E^{\prime \prime}}, N \uparrow_{E^{\prime \prime}}\right)$. Now, we need to show that every covering for $\left(M^{*} \uparrow_{E^{\prime \prime}}, N \uparrow_{E^{\prime \prime}}\right)$ is also a packing. Let $(A, B)$ be a covering for $\left(M^{*} \uparrow_{E^{\prime \prime}}, N \uparrow_{E^{\prime \prime}}\right)$. Observe that $\left(A \backslash U_{i}, B \backslash U_{i}\right)$ is a covering for $\left(M^{*} \uparrow_{E^{\prime}}, N \uparrow_{E^{\prime}}\right)$. In the proof of the lemma 4.3.2 it is shown that the set $E^{\prime}$ is a critical set for $\left(M^{*}, N\right)$. Thus, $\left(A \backslash U_{i}, B \backslash U_{i}\right)$ is also a packing for $\left(M^{*} \uparrow_{E^{\prime}}, N \uparrow_{E^{\prime}}\right)$. Since $B \backslash U_{i}$ is a base of $N \uparrow_{E^{\prime}}$ and $F_{i} \subseteq \mathscr{F}(K)$, this implies that $B \cap U_{i}=\emptyset$ because otherwise it contradict with $B$ is independent in $N$. Therefore $U_{i} \subseteq A$. Since $A \backslash U_{i}$ is a base of $\left.M^{*}\right|_{E^{\prime}}$ and $U_{i}$ is a base of $M^{*} \uparrow_{\{i\}}$, we have $A$ is a base of $M^{*} \uparrow_{E^{\prime \prime}}$. Thus, $(A, B)$ is also a packing for $\left(M^{*} \uparrow_{E^{\prime \prime}}, N \uparrow_{E^{\prime \prime}}\right)$. This complete the proof that $E^{\prime \prime}$ is a critical set for $\left(M^{*}, N\right)$.

\subsection{Proof of Main Results}

\subsubsection{Infinite Exchange Chain}

Throughout this section we assume that $M$ and $N$ are matroids on a common ground set $E$. 
Let $I$ and $J$ be independent in $M$ and $N$, respectively. An $(I, J)$-exchange $M$-chain is a finite sequence $\left\langle e_{1}, e_{2}, \ldots, e_{n}\right\rangle$ of elements of $E$ such that for each $i \in\{1,2, \ldots, n-1\}$ the elements $e_{i}$ and $e_{i+1}$ are distinct and:

- if $i$ is odd, then there exists a circuit $C_{e_{i}}$ of $M$ with $e_{i}, e_{i+1} \in C_{e_{i}} \subseteq I \cup\left\{e_{i}\right\}$;

- if $i$ is even, then there exists a circuit $C_{e_{i}}$ of $N$ with $e_{i}, e_{i+1} \in C_{e_{i}} \subseteq J \cup\left\{e_{i}\right\}$.

We say that such a chain is from $e_{1}$ to $e_{n}$. Note that such $C_{e_{i}}$ is unique and we call it the $(I, J)$ fundamental circuit of $e_{i}$ for each $i \in\{1,2, \ldots, n-1\}$. If $\left\langle e_{1}, e_{2}, \ldots\right\rangle$ is an infinite sequence of elements of $E$ such that for each $i \in \mathbb{N}$ the finite initial segment $\left\langle e_{1}, e_{2}, \ldots, e_{i}\right\rangle$ is an $(I, J)$ exchange $M$-chain, then we call the sequence $\left\langle e_{1}, e_{2}, \ldots\right\rangle$ an infinite $(I, J)$-exchange $M$-chain. An (infinite) $(I, J)$-exchange $N$-chain is defined analogously with the words "even" and "odd" interchanged. An (infinite) $(I, J)$-exchange chain refers to either of these notions.

Definition 4.4.1. Let $I$ and $J$ be independent in $M$ and $N$, respectively. An (infinite) $(I, J)$ exchange string is an (infinite) $(I, J)$-exchange chain $\left\langle e_{1}, e_{2}, \ldots\right\rangle$ such that for each $i \in \mathbb{N}$, the finite initial segment $\left\langle e_{1}, e_{2}, \ldots, e_{i}\right\rangle$ is the shortest $(I, J)$-exchange chain from $e_{1}$ to $e_{i}$.

Note that if $\left\langle e_{1}, e_{2}, \ldots\right\rangle$ is an infinite $(I, J)$-exchange string, then for each $i \in \mathbb{N}$, the $(I, J)$ fundamental circuit of $e_{i}$ does not contain any $e_{j}$ for $j>i+1$.

Definition 4.4.2. A covering $(I, J)$ for $(M, N)$ is special if and only if $I$ and $J$ are disjoint and the followings hold:

1. There exists a partition of $E=\bigsqcup_{k \in K} E_{k}$ such that

$$
M=\bigoplus_{k \in K} M_{k}
$$

with each $M_{k}$ being a matroid on the set $E_{k}$.

2. There exists a partition of $E=\bigsqcup_{l \in L} E_{l}^{\prime}$ such that

$$
N=\bigoplus_{l \in L} N_{l}
$$


with each $N_{l}$ being a matroid on the set $E_{l}^{\prime}$.

3. For any circuit $C$ of $M$, either $C$ is finite or $\left(E_{k} \backslash C\right) \cap J$ is finite, for the unique $k \in K$ such that $C \subseteq E_{k}$.

4. For any circuit $C$ of $N$, either $C$ is finite or $\left(E_{l}^{\prime} \backslash C\right) \cap I$ is finite, for the unique $l \in L$ such that $C \subseteq E_{l}^{\prime}$.

The partition $E=\bigsqcup_{k \in K} E_{k}$ is called I-special or e-special for $e \in I$ and the partition $E=\bigsqcup_{l \in L} E_{l}^{\prime}$ is called $J$-special or $e$-special when $e \in J$.

Remark 4.4.3. Let $M$ be finitary and $N=\bigoplus_{l \in L} N_{l}$ with each $N_{l}$ being a uniform matroid of rank finite. If $(M, N)$ has a covering, then it also has a special covering.

Definition 4.4.4. We say $a \in E$ is essential for $(M, N)$ if and only if $(M, N)$ has a covering and $(M, N) /\{a\}$ has no covering.

Lemma 4.4.5. If $a \in E$ is essential for $(M, N)$, then for any covering $(I, J)$ of $(M, N)$ we have $a \in \operatorname{cl}_{M}(I) \cap \operatorname{cl}_{N}(J)$.

Proof. By symmetry it suffices to show that $a \in \mathrm{cl}_{M}(I)$. Suppose, for a contradiction, that $a \notin \mathrm{cl}_{M}(I)$. So, $a \in J$ and $I \cup\{a\}$ is independent in $M$. Then, $(I, J \backslash\{a\})$ is a covering for $(M, N) /\{a\}$ which is a contradiction with the assumption.

Lemma 4.4.6. Let $(I, J)$ be a covering for $(M, N)$ and $S=\left\langle x_{0}, x_{1}, x_{2}, \cdots, x_{n}\right\rangle$ an $(I, J)$-exchange chain from $x_{0}$ to $x_{n}$ with $x_{n} \in J$. If $x_{n} \notin \mathrm{cl}_{M}(I)$, then $\left(I^{\prime}, J^{\prime}\right)$ is a covering for $(M, N)$ in which $I^{\prime}=I \cup(S \cap J) \backslash(S \cap I)$ and $J^{\prime}=J \cup(S \cap I) \backslash(S \cap J)$. (See figure 4.4.1)

Proof. We show by induction on $k \in \mathbb{N}$ that we have the followings:

1. If

$$
I_{k}=I \cup\left\{x_{n}, x_{n-2}, \ldots, x_{n-2 k}\right\} \backslash\left\{x_{n-1}, x_{n-3} \ldots, x_{n-2 k+1}\right\}
$$




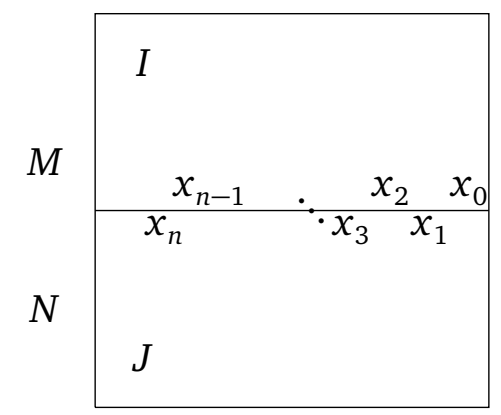

(a) before the exchange

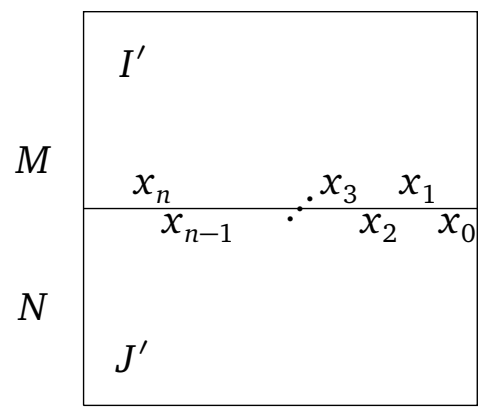

(b) after the exchange

Figure 4.4.1: The $(I, J)$-exchange chain $\left\langle x_{0}, x_{1}, x_{2}, \cdots, x_{n}\right\rangle$ and the covering $(I, J)$ and $\left(I^{\prime}, J^{\prime}\right)$ for $(M, N)$.

and

$$
J_{k}=J \cup\left\{x_{n-1}, x_{n-3}, \ldots, x_{n-2 k+1}\right\} \backslash\left\{x_{n}, x_{n-2}, \ldots, x_{n-2 k}\right\},
$$

then $\left(I_{k}, J_{k}\right)$ is a covering for $(M, N)$ and $x_{n-2 k-1} \notin \operatorname{cl}_{N}\left(J_{k}\right)$.

2. If

$$
I_{k}=I \cup\left\{x_{n}, x_{n-2}, \ldots, x_{n-2 k}\right\} \backslash\left\{x_{n-1}, x_{n-3} \ldots, x_{n-2 k-1}\right\}
$$

and

$$
J_{k}=J \cup\left\{x_{n-1}, x_{n-3} \ldots, x_{n-2 k-1}\right\} \backslash\left\{x_{n}, x_{n-2}, \ldots, x_{n-2 k}\right\},
$$

then $\left(I_{k}, J_{k}\right)$ is a covering for $(M, N)$ and $x_{n-2 k-2} \notin \mathrm{cl}_{M}\left(I_{k}\right)$

First observe that (1) implies (2). So, it in enough to show that the case (1) is true for $k=0$. When $k=0$, we have $I_{0}=I \cup\left\{x_{n}\right\}$ and $J_{0}=J \backslash\left\{x_{n}\right\}$. Since by the assumption $x_{n} \notin \mathrm{cl}_{M}(I)$, we have $\left(I_{0}, J_{0}\right)$ is a covering for $(M, N)$. Also, we have $x_{n-1} \notin \mathrm{cl}_{N}\left(J_{0}\right)$ because $x_{n} \in C_{x_{n-1}}$, in which $C_{x_{n-1}}$ is the $(I, J)$-fundamental circuit of $x_{n-1}$ and $x_{n} \notin J_{0}$. This implies that the case (1) is true for $k=0$ and this completes the proof of the induction. Now, since for any $k \in \mathbb{N},\left(I_{k}, J_{k}\right)$ is a covering for $(M, N)$ we have $\left(I^{\prime}, J^{\prime}\right)$ is a covering for $(M, N)$ in which $I^{\prime}=I \cup(S \cap J) \backslash(S \cap I)$ and $J^{\prime}=J \cup(S \cap I) \backslash(S \cap J)$. 


\subsubsection{Proof of Theorems 4.1.9, 4.1.10, and Corollary 4.1.11}

Lemma 4.4.7. Suppose $a \in E$ is essential for $(M, N)$. Let $(I, J)$ be a covering for $(M, N)$ and $A$ be the set of all elements of $E$ to which there exists an $(I, J)$-exchange chain starting at a. Then $A \subseteq \mathrm{cl}_{M}(I)$ and $A \subseteq \mathrm{cl}_{N}(J)$.

Proof. Suppose, for a contradiction, that there exists some

$$
b \in A \backslash\left(\mathrm{cl}_{M}(I) \cup \mathrm{cl}_{N}(J)\right) .
$$

By symmetry, we can assume that $a \in I$. Since $b \in A$, there exists an $(I, J)$-exchange $N$-chain from $a$ to $b$. Let

$$
S=\left\{x_{0}, x_{1}, x_{2}, \cdots, x_{n}\right\}
$$

with $x_{0}=a$ and $x_{n}=b$, be the shortest $(I, J)$-exchange $N$-chain from $a$ to $b$. Let

$$
I^{\prime}=I \cup(S \cap J) \backslash(S \cap I),
$$

and

$$
J^{\prime}=J \cup(S \cap I) \backslash(S \cap J)
$$

By the lemma 4.4.6 and the choice of $b$ we have $\left(I^{\prime}, J^{\prime}\right)$ is a covering for $(M, N)$. See figure 4.4.2 for the case when $b \in J$. Let $\mathscr{C}$ be the family of all circuits $C$ of $M$ such that $C \subseteq I \cup S$. For each $C \in \mathscr{C}$, let

$$
\operatorname{so}(C)=\min \left\{i \in\{0,1, \ldots, n\}: i \text { is odd and } x_{i} \in C\right\}
$$




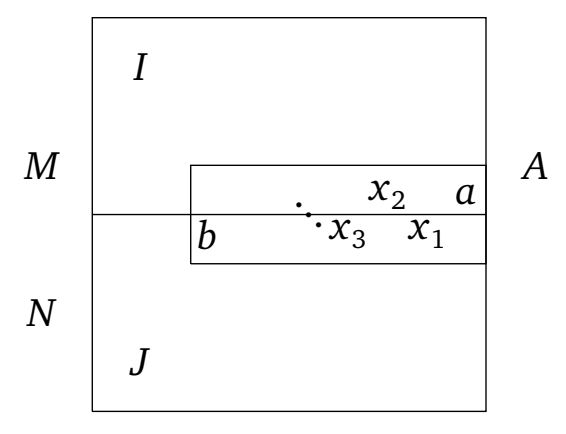

(a) before the exchange

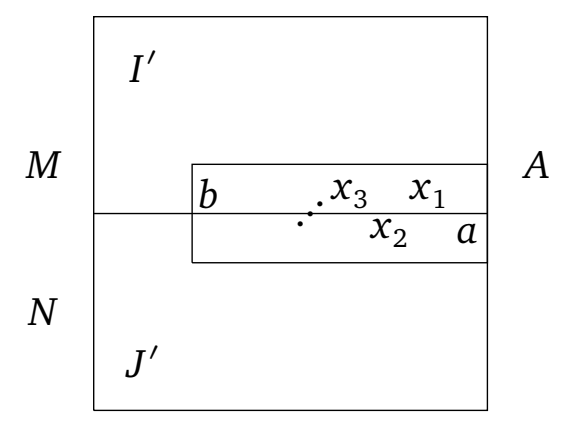

(b) after the exchange

Figure 4.4.2: A shortest $N-(I, J)$-exchange chain $\left\langle a, x_{1}, x_{2}, x_{3}, \cdots, b\right\rangle$ from $a$ to $b$ for the case when $b \in A \backslash \operatorname{cl}_{M}(I)$.

be the smallest odd index $i$ of $x_{i}$ in $C$ (we will call it the smallest odd index of $C$ ) and

$$
\operatorname{le}(C)=\max \left\{i \in\{0,1, \ldots, n\}: i \text { is even and } x_{i} \in C\right\}
$$

be the largest even such index. Let

$$
\mathscr{C}^{\prime}=\{C \in \mathscr{C}: \operatorname{le}(C)<\operatorname{so}(C)\}
$$

By the lemma 4.4.5, we have $a \in \mathrm{cl}_{M}\left(I^{\prime}\right)$ and so there exists a circuit $C^{M}$ of $M$ such that

$$
a \in C^{M} \subseteq I^{\prime} \cup\{a\}
$$

Since $C^{M} \in \mathscr{C}^{\prime}$ it follows that $\mathscr{C}^{\prime} \neq \varnothing$. Let $C^{\prime}$ be a circuit in $\mathscr{C}^{\prime}$ for which the smallest odd index is as large as possible and let $i=\operatorname{so}\left(C^{\prime}\right)$. If $n$ is odd, then $b \notin \mathrm{cl}_{M}(I)$ implying that $i<n$.

There is circuit $C_{i}$ of $M$ that $x_{i} \in C_{i} \subseteq I \cup\left\{x_{i}\right\}$ and $x_{i+1} \in C_{i}$. In particular $C_{i} \notin \mathscr{C}^{\prime}$ implying that $C_{i} \neq C^{\prime}$. By eliminating $x_{i}$ from $C_{i}$ and $C^{\prime}$ we get a circuit $C^{\prime \prime}$ of $M$ that $C^{\prime \prime} \subseteq\left(C_{i} \cup C^{\prime}\right) \backslash\left\{x_{i}\right\}$. Since $S$ is the shortest $(I, J)$-exchange $N$-chain from $a$ to $b$, it follows that $C_{i}$ contains no $x_{j}$ with even $j>i+1$ and consequently we have $C^{\prime \prime} \in \mathscr{C}^{\prime}$. Since $\operatorname{so}\left(C^{\prime \prime}\right)>i$, we have a contradiction. 
Lemma 4.4.8. Let $(I, J)$ be a special covering for $(M, N)$ and $S=\left\langle e_{0}, e_{1}, e_{2}, \ldots\right\rangle$ be an infinite $(I, J)$-exchange chain. Then there is an infinite $(I, J)$-exchange string starting at $e_{0}$ that is a sub-sequence of $S$.

Proof. Without loss of generality, we can assume that $e_{0} \in I$. Then we have

$$
\left\{e_{0}, e_{2}, e_{4}, \cdots\right\} \subseteq I \text { and }\left\{e_{1}, e_{3}, e_{5}, \cdots\right\} \subseteq J
$$

Let $i \in \mathbb{N}, C_{e_{i}}$ be the $(I, J)$-fundamental circuit of $e_{i}$, and $E=\bigsqcup_{k \in K} E_{k}$ be an $e_{i}$-special partition of E.

1. If $C_{e_{i}}$ is finite, then let

$$
\alpha_{i}=\max \left\{j \in \mathbb{N}: e_{j} \in C_{e_{i}}\right\} .
$$

2. Otherwise, then let

$$
\alpha_{i}=\max \left\{j \in \mathbb{N}: e_{j} \in C_{e_{i}} \text { with } e_{j-1} \in E_{k} \backslash C_{e_{i}} \text { where } E_{k} \text { is such that } C_{e_{i}} \subseteq E_{k}\right\} .
$$

Let $\left\langle\beta_{i}: i \in \mathbb{N}\right\rangle$ be the sequence defined inductively by

$$
\begin{gathered}
\beta_{0}=\alpha_{0} \\
\beta_{i+1}=\alpha_{\beta_{i}+1}
\end{gathered}
$$

for each $i \in \mathbb{N}$. Now consider the circuit $C_{e_{0}}$ and from the sequence $S$, remove any $e_{k}$ such that $0<k<\beta_{0}$. The sub-sequence $S^{\prime}=\left\langle e_{0}, e_{\beta_{0}}, e_{\beta_{1}}, \cdots\right\rangle$ of elements of $S$ is an $(I, J)$-exchange chain such that for each $i \in \mathbb{N}$, the circuit $C_{e_{\beta_{i}}}$ contains no $e_{\beta_{j}}$ for $j>i+1$. This implies that $S^{\prime}=\left\langle e_{0}, e_{\beta_{1}}, e_{\beta_{2}}, \cdots\right\rangle$ is an $(I, J)$-exchange string starting at $e_{0}$.

Lemma 4.4.9. Let $(I, J)$ be a special covering, $a \in E$ essential for $(M, N)$, and $A$ the set of all elements of $E$ to which there exist an $(I, J)$-exchange chain starting at a. If $\left(I^{\prime}, J^{\prime}\right)$ is a covering for $\left(M \uparrow_{A}, N \uparrow_{A}\right)$, then $A \subseteq \mathrm{cl}_{M}\left(I^{\prime}\right)$ and $A \subseteq \mathrm{cl}_{N}\left(J^{\prime}\right)$. 
Proof. Let $\left(I^{\prime}, J^{\prime}\right)$ be a covering for $\left(M \uparrow_{A}, N \uparrow_{A}\right)$. We show that $A \subseteq \mathrm{cl}_{M}\left(I^{\prime}\right)$ and the proof of $A \subseteq \operatorname{cl}_{N}\left(J^{\prime}\right)$ follows by a similar argument.

Suppose, for a contradiction, that there is some $b \in A \backslash \mathrm{cl}_{M}\left(I^{\prime}\right)$. We assume that $b \in I$ and the proof for the case when $b \in J$ can be obtained by a similar idea and construction.

We claim that there exists an infinite $(I, J)$-exchange chain $\left\langle x_{0}, x_{1}, x_{2}, x_{3}, \cdots\right\rangle$ with $x_{0}=b$ such that

$$
\left\{x_{0}, x_{2}, x_{4}, \cdots\right\} \subseteq J^{\prime} \cap I
$$

and

$$
\left\{x_{1}, x_{3}, x_{5}, \cdots\right\} \subseteq I^{\prime} \cap J .
$$

To prove this claim we define a bipartite graph $G_{1}$ on vertex sets

$$
P=I \cap J^{\prime} \text { and } Q=J \cap I^{\prime}
$$

as follows: For every vertex $y \in P$, let $C_{y} \subseteq J \cup\{y\}$ be the (I,J)-fundamental circuit of $y$. In the graph $G_{1}$, we connect the vertex $y$ to all elements in $C_{y} \cap I^{\prime}$. We define a bipartite graph $G_{2}$ on vertex set

$$
Q=J \cap I^{\prime} \text { and } \quad P \backslash\{b\}=\left(I \cap J^{\prime}\right) \backslash\{b\}
$$

as follows: For every vertex $x \in Q$, let $C_{x} \subseteq I \cup\{x\}$ be the $(I, J)$-fundamental circuit of $x$. In graph $G_{2}$, we connect the vertex $x$ to all elements in $\left(C_{x} \cap J^{\prime}\right) \backslash\{b\}$.

In the followings, we show that in the graph $G_{1}$, the vertex set $P$ is matchable into the vertex set $Q$ and in the graph $G_{2}$, the vertex set $Q$ is matchable into the vertex set $P \backslash\{b\}$.

First consider the graph $G_{1}$ and the partition of the vertex set 
$G_{1}$

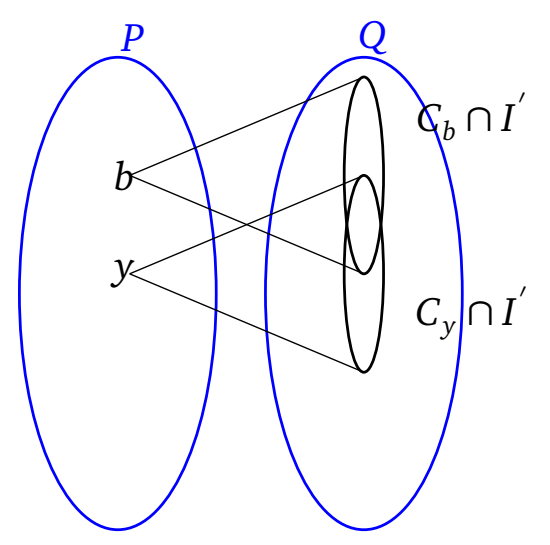

$G_{2}$

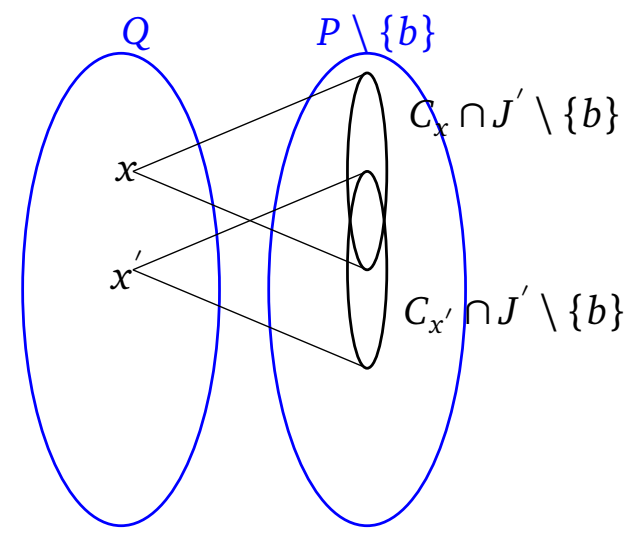

Figure 4.4.3: Graphs $G_{1}$ and $G_{2}$

$$
P=\bigsqcup_{l \in L} P_{l} \quad \text { with } \quad P_{l}=E_{l}^{\prime} \cap P
$$

where $E=\bigsqcup_{l \in L} E_{l}^{\prime}$ is a $J$-special. Since $P=\bigsqcup_{l \in L} P_{l}$ is a partition of the vertex set $P$ and for any $l, l^{\prime} \in L$, the vertex sets $E\left[P_{l}\right]$ and $E\left[P_{l^{\prime}}\right]$ are disjoint, to show that $P$ is matchable into the vertex set $Q$, it is enough to show that for any arbitrary $l \in L$, the vertex set $P_{l}$ is matchable into $E\left[P_{l}\right]$. Now, let $l \in L$, then we have two possibilities:

1. either every $y \in P_{l}$ has finite degree.

2. or the set $P_{l}$ is finite.

This is true because, if (1) does not hold, then for some $y \in P_{l}$, the circuit $C_{y}$ which is the $(I, J)$-fundamental circuit of $y$ is infinite. Since $(I, J)$ is a special cover, we have $\left(E_{l}^{\prime} \backslash C_{y}\right) \cap I$ is finite and since $P_{l} \subseteq\left(E_{l}^{\prime} \backslash C_{y}\right) \cap I$, this implies that the set $P_{l}$ is finite.

By symmetry, consider the graph $G_{2}$ and the partition of the vertex set

$$
Q=\bigsqcup_{k \in K} Q_{k} \quad \text { with } \quad Q_{k}=E_{k} \cap Q
$$

where $E=\bigsqcup_{k \in K} E_{k}$ is an $I$-special. Since $Q=\bigsqcup_{k \in K} Q_{k}$ is a partition of the vertex set $Q$ and for any $k, k^{\prime} \in K$, the vertex sets $E\left[P_{k}\right]$ and $E\left[P_{k^{\prime}}\right]$ are disjoint, to show that $Q$ is matchable into 
the vertex set $P \backslash\{b\}$, it is enough to show that for any arbitrary $k \in K$, the vertex set $Q_{k}$ is matchable into $E\left[Q_{k}\right]$. Now, let $k \in K$, by a similar argument as above we have:

1. either every $x \in Q_{k}$ has finite degree.

2. or the set $Q_{k}$ is finite.

By symmetry, it suffices to show that in the graph $G_{2}$, for every $k \in K$, the vertex set $Q_{k}$ is matchable into $E\left[Q_{k}\right]$. Let $k \in K$.

By Theorem 4.1.3, it is enough to show that for every finite set $F \subseteq Q_{k}$ we have $|F| \leq|E[F]|$. Suppose, for a contradiction, that there is $F_{0} \subseteq Q_{k}$ with $\left|F_{0}\right|>\left|E\left(F_{0}\right)\right|$. In the following we show that this leads to a contradiction.

Let $\mathscr{F}$ be the family of all pairs $(F, \mathscr{C})$ where $F$ is a finite set,

$$
F \subseteq Q_{k}
$$

and

$$
\mathscr{C}=\left\{C_{x}: x \in F\right\}
$$

such that $C_{x}$ is a circuit of $M$ and

$$
\begin{gathered}
x \in C_{x}, \\
C_{x} \cap F=\{x\}, \\
C_{x} \subseteq Q_{k} \cup I,
\end{gathered}
$$

and

$$
|F|>|G(F, \mathscr{C})|
$$

where

$$
G(F, \mathscr{C})=\left\{y \in P \backslash\{b\}: y \in C_{x} \text { for some } C_{x} \in \mathscr{C}\right\}
$$


and

$$
G^{\prime}(F, \mathscr{C})=G(F, \mathscr{C}) \cup\left\{z \in Q \backslash F: z \in C_{x} \text { for some } C_{x} \in \mathscr{C}\right\}
$$

is independent in $M$.

Observe that $\mathscr{F} \neq \emptyset$ since $\left(F_{0}, \mathscr{C}_{0}\right) \in \mathscr{F}$ in which

$$
\mathscr{C}_{0}=\left\{C_{x}: x \in F_{0} \text { and } C_{x} \text { is the }(I, J)-\text { fundamental circuit of } x\right\} .
$$

Let $(\hat{F}, \hat{\mathscr{C}}) \in \mathscr{F}$ be such that $|\hat{F}| \leq|F|$ for any $(F, \mathscr{C}) \in \mathscr{F}$. Since for any $x \in \hat{F}$, there is $C_{x}$, the $(I, J)$-fundamental circuit of $x$, which

$$
C_{x} \cap G(\stackrel{\wedge}{F}, \hat{\mathscr{C}}) \neq \emptyset
$$

and

$$
|\hat{F}|>|G(\stackrel{\wedge}{F}, \hat{\mathscr{C}})|
$$

there is some $y_{0} \in G(\hat{F}, \hat{\mathscr{C}})$ for which there are at least two elements $x, x^{\prime} \in \hat{F}$ with

$$
y_{0} \in C_{x} \cap C_{x^{\prime}}
$$

Fix $x_{0} \in \hat{F}$ such that $y_{0} \in C_{x_{0}}$ and let $H^{\prime} \subseteq \hat{F} \backslash\left\{x_{0}\right\}$ be the set of all $x \in \hat{F} \backslash\left\{x_{0}\right\}$ such that $y_{0} \in C_{x}$. Eliminate $y_{0}$ from all pairs of circuits $C_{x_{0}}$ and $C_{x}$ for all $x \in H^{\prime}$ as follows: For each $x \in H^{\prime}$, let $C_{x}^{\prime}$ be a circuit of $M$ such that

$$
C_{x}^{\prime} \subseteq\left(C_{x} \cup C_{x_{0}}\right) \text { and } y_{0} \notin C_{x}^{\prime}
$$

Let $H=F \backslash\left\{x_{0}\right\}$ and $C_{x}^{\prime}=C_{x}$ for any $x \in H \backslash H^{\prime}$. We claim that $\left(H, \mathscr{C}^{\prime}\right) \subseteq \mathscr{F}$ where $\mathscr{C}^{\prime}=\left\{C_{x}^{\prime}: x \in H\right\}$.

We first need to show that $x \in C_{x}^{\prime}$ for any $x \in H$. If $x \in H \backslash H^{\prime}$, it is obvious. Let $x \in H^{\prime}$ 
and suppose, for a contradiction, that $x \notin C_{x}^{\prime}$. Since

$$
C_{x}^{\prime} \subseteq\left(C_{x} \cup C_{x_{0}}\right) \backslash\left\{y_{0}\right\}
$$

and $G^{\prime}(F, \mathscr{C})$ is independent in $M$, we must have $x_{0} \in C_{x}^{\prime}$. Now elimination of $x_{0}$ from the circuits $C_{x}^{\prime}$ and $C_{x_{0}}$ gives a circuit of $M$ in $G^{\prime}(F, \mathscr{C})$ which is a contradiction with $G^{\prime}(F, \mathscr{C})$ is independent in $M$. Obviously

$$
C_{x}^{\prime} \cap H=\{x\} \text { and } C_{x}^{\prime} \subseteq Q_{k} \cup I .
$$

Also, we have

$$
|H|>\left|G\left(H, \mathscr{C}^{\prime}\right)\right|
$$

because

$$
|F|>|G(F, \mathscr{C})|
$$

and

$$
|H|=|F|-1
$$

and

$$
\left|G\left(H, \mathscr{C}^{\prime}\right)\right| \leqslant G(F, \mathscr{C})-1
$$

because

$$
G\left(H, \mathscr{C}^{\prime}\right) \subseteq G(F, \mathscr{C}) \backslash\left\{y_{0}\right\}
$$

It remains to show that $G^{\prime}\left(H, \mathscr{C}^{\prime}\right)$ is independent in $M$. First, note that

$$
G^{\prime}\left(H, \mathscr{C}^{\prime}\right) \subseteq G^{\prime}(F, \mathscr{C}) \cup\left\{x_{0}\right\} \backslash\left\{y_{0}\right\}
$$

Suppose, for a contradiction, that there is a circuit $C$ of $M$ such that $C \subseteq G^{\prime}\left(H, \mathscr{C}^{\prime}\right)$. Observe that $x_{0} \in C$ and $y_{0} \notin C$. So, $C$ and $C_{x_{0}}$ are two distinct circuits of $M$ containing $x_{0}$. Now, by 
eliminating $x_{0}$ from the circuits $C$ and $C_{x_{0}}$, we get a circuit of $M$ inside $G^{\prime}(F, \mathscr{C})$ which is a contradiction with $G^{\prime}(F, \mathscr{C})$ is independent in $M$. Hence,

$$
\left(H, \mathscr{C}^{\prime}\right) \subseteq \mathscr{F} \text { with }|H|<|F|
$$

This is a contradiction with the definition of $\hat{F}$. So, we proved that in the graph $G_{2}$, the vertex set $Q_{k}$ is matchable into $E\left[Q_{k}\right]$ for any $k \in K$. This implies that in the graph $G_{2}, Q$ is matchable into the vertex set $P \backslash\{b\}$ and similarly, in the graph $G_{1}$, the vertex set $P$ is matchable into the vertex set $Q$.

Thus, there exists an infinite $(I, J)$-exchange chain $S=\left\{x_{0}, x_{1}, x_{2}, x_{3}, \cdots\right\}$ with $x_{0}=b$ such that

$$
\left\{x_{0}, x_{2}, x_{4}, \cdots\right\} \subseteq J^{\prime} \cap I
$$

and

$$
\left\{x_{1}, x_{3}, x_{5}, \cdots\right\} \subseteq I^{\prime} \cap J
$$

Let $x_{j} \in S$ and $L$ be an $(I, J)$-exchange chain from $a$ to $x_{j}$. Now, let

$$
S^{\prime}=S \backslash\left\{b, x_{1}, \cdots, x_{j-1}\right\}=\left\{x_{j}, x_{j+1}, x_{j+2}, \cdots\right\}
$$

and $S^{\prime \prime}=S^{\prime} \cup L$. By the lemma 4.4.8, there exists an infinite $(I, J)$-exchange string

$$
S^{\prime \prime \prime}=\left\{z_{0}, z_{1}, z_{2}, \cdots\right\}
$$

with $z_{0}=a$ that is a sub-sequence of $S$. Let

$$
I^{\prime \prime}=I \cup\left(S^{\prime \prime \prime} \cap J\right) \backslash\left(S^{\prime \prime \prime} \cap I\right),
$$




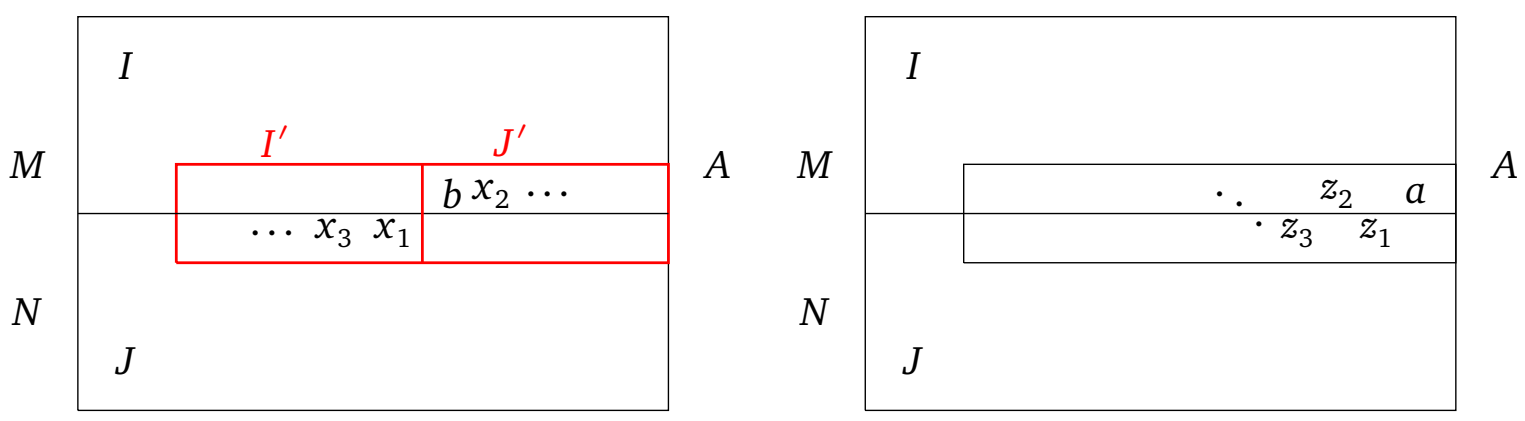

Figure 4.4.4: The sets $S=\left\{b, x_{1}, x_{2}, x_{3}, \cdots\right\}$ and $S^{\prime \prime \prime}=\left\{a, z_{1}, z_{2} \cdots\right\}$ for the case $a \in I$.

and

$$
J^{\prime \prime}=J \cup\left(S^{\prime \prime \prime} \cap I\right) \backslash\left(S^{\prime \prime \prime} \cap J\right)
$$

We show that $\left(I^{\prime \prime}, J^{\prime \prime}\right)$ is a covering for $(M, N)$. Without loss of generality, we can assume that $a \in I$ and follow the next argument. The proof for the case that $a \notin I$ follows by a similar argument and replacing odd and even indices.

By symmetry, it is enough to show that $I^{\prime \prime}$ is independent in $M$. Suppose, for a contradiction, that there is a circuit $C$ of $M$ such that $C \subseteq I^{\prime \prime}$. Observe that $C \cap\left\{z_{1}, z_{3}, z_{5}, \cdots\right\} \neq \emptyset$. Since the partition $E=\bigsqcup_{k \in K} E_{k}$ is an $I$-special, the set $C \cap\left\{z_{1}, z_{3}, z_{5}, \cdots\right\}$ is finite because:

1. If $C$ is finite, then clearly $C \cap\left\{z_{1}, z_{3}, z_{5}, \cdots\right\}$ is finite.

2. If $C$ is infinite and $z_{r} \in C$ for some $r \in\{1,3,5, \cdots\}$, then $\left(E_{k} \backslash C_{z_{r}}\right) \cap J$ is finite for the unique $k \in K$ that $C_{z_{r}} \subseteq K$. Since $C \cap\left\{z_{1}, z_{3}, z_{5}, \cdots\right\} \subseteq\left(E_{k} \backslash C_{z_{r}}\right) \cap J$, we have $C \cap\left\{z_{1}, z_{3}, z_{5}, \cdots\right\}$ is finite.

Let $i$ to be the largest odd index that $z_{i} \in C$ and $I^{*}=I \backslash\left\{z_{i+1}\right\}$. Observe that the pair $\left(I^{*}, J\right)$ is independent in $(M, N)$. Since $z_{i} \notin \mathrm{cl}_{M}\left(I^{*}\right)$, by the lemma 4.4 .6 we have $(\widehat{I}, \widehat{J})$ is a pair of independent sets for $(M, N)$ in which

$$
\widehat{I}=I^{*} \cup\left\{z_{1}, z_{3}, z_{5}, \cdots, z_{i}\right\} \backslash\left\{z_{0}, z_{2}, z_{4}, \cdots, z_{i-1}\right\}
$$


and

$$
\widehat{J}=J \cup\left\{z_{0}, z_{2}, z_{4}, \cdots, z_{i-1}\right\} \backslash\left\{z_{1}, z_{3}, z_{5}, \cdots, z_{i}\right\} .
$$

But, the circuit $C \subseteq \widehat{I}$ which is a contradiction with $\widehat{I}$ is independent in $M$. So the proof that $\left(I^{\prime \prime}, J^{\prime \prime}\right)$ is a covering for $(M, N)$ is completed.

Since $a$ is is essential for $(M, N)$, by the lemma 4.4.5, we have

$$
\mathrm{a} \in \operatorname{cl}_{M}\left(I^{\prime \prime}\right) \cap \mathrm{cl}_{N}\left(J^{\prime \prime}\right)
$$

and since we considered the case that $a \in I$, we have $a \notin I^{\prime \prime}$. So, there is a circuit $C$ of $M$ such that $a \in C \subseteq I^{\prime \prime} \cup\{a\}$. Observe that $C \cap\left\{z_{1}, z_{3}, z_{5}, \cdots\right\} \neq \emptyset$. Here we get a contradiction again by using the fact that the partition $E=\bigsqcup_{k \in K} E_{k}$ is an $I$-special and applying the lemma 4.4.6 and the proof completes.

Now we are ready to prove Theorem 4.1.9.

Proof of Theorem 4.1.9. Let $A$ be the set of all elements of $E$ to which there exist an $(I, J)$ exchange chain starting at $a$. We show that $A$ is a critical set. Suppose not, then there is a covering $\left(I^{\prime}, J^{\prime}\right)$ for $\left(M \uparrow_{A}, N \uparrow_{A}\right)$ such that $I^{\prime} \cap J^{\prime} \neq \emptyset$. Let $b \in I^{\prime} \cap J^{\prime}$. There is an $(I, J)$ exchange chain from $a$ to $b$. So, there is covering $\left(I^{\prime \prime}, J^{\prime \prime}\right)$ for $\left(M \uparrow_{A}, N \uparrow_{A}\right)$ with $a \in I^{\prime \prime} \cap J^{\prime \prime}$. Now, $\left(I^{\prime \prime} \backslash\{a\}, J^{\prime \prime}\right)$ is a covering for $\left(M \uparrow_{A}, N \uparrow_{A}\right)$. By the lemma 4.4.9, we have $A \subseteq \operatorname{cl}_{M}\left(I^{\prime \prime} \backslash\{a\}\right)$, and hence $a \in \mathrm{cl}_{M}\left(I^{\prime \prime} \backslash\{a\}\right)$ which is a contradiction.

Lemma 4.4.10. Suppose $(M, N)$ has a covering and $\mathscr{A}=\{A \subseteq E: A$ is ciritical for $(M, N)\}$. Then $\cup \mathscr{A}$ is critical for $(M, N)$.

Proof. Let $\mathscr{A}=\{A \subseteq E: A$ is ciritical for $(M, N)\}$ and $K=\bigcup \mathscr{A}$. Since $(M, N)$ has a covering, $\left(M \uparrow_{K}, N \uparrow_{K}\right)$ has a covering. So, it is enough to show that every covering of $\left(M \uparrow_{K}, N \uparrow_{K}\right)$ is also a packing. Suppose, for a contradiction, that there is a covering $(I, J)$ for $\left(M \uparrow_{K}, N \uparrow_{K}\right)$ that $I \cap J \neq \emptyset$. Let $b \in I \cap J$. There is some $A \in \mathscr{A}$ such that $b \in A$. Observe that $(I \cap A, J \cap A)$ 
is a covering for $\left(M \uparrow_{A}, N \uparrow_{A}\right), b \in I \cap A$, and $b \in J \cap A$ which implies that $(I \cap A, J \cap A)$ is not a packing for $\left(M \uparrow_{A}, N \uparrow_{A}\right)$. This is a contradiction with $A$ being a critical set for $(M, N)$.

Proof of Theorem 4.1.10. Let $\mathscr{A}=\{A \subseteq E: A$ is ciritical for $(M, N)\}$. Since $(M, N)$ has a covering, by the lemma 4.4.10, we have $K=\bigcup \mathscr{A}$ is critical for $(M, N)$. Therefore $K$ is a maximal critical set for $(M, N)$.

Proof of Corollary 4.1.11. Let $a \in E^{\prime}$. Since $a$ is essential for $(M, N)$ by Theorem 4.1.9, there is a critical set $A_{a}$ such that $a \in A_{a}$. Now, let $K=\bigcup_{a \in E^{\prime}} A_{a}$. By the lemma 4.4.10, $K$ is a critical set for $(M, N)$ and also $E^{\prime} \subseteq K$.

Remark 4.4.11. If $(M, N)$ has no covering, then the lemma 4.4.10 may not be true. For example, let $M$ be the algebraic cycle matroid of a double ray on an edge set $E$ and $N$ a matroid with the family of independent sets to be the empty set. First observe that $(M, N)$ has no covering. Now, let $\mathscr{A}=\{A \subseteq E: A$ is ciritical for $(M, N)\}$. Then $\bigcup \mathscr{A}=E$ which is not a critical set for $(M, N)$ because $(M, N)$ has no covering.

Remark 4.4.12. If $\mathscr{F}=\left(F_{i}: i \in I\right)$ is a family of sets and $\left\langle A_{\alpha}: \alpha<\gamma\right\rangle$ a sequence of critical sets for $\mathscr{F}$ such that $A_{\alpha} \subseteq A_{\beta}$ for every $\alpha \leq \beta<\gamma$, then $K=\bigcup_{\alpha<\gamma} A_{a}$ is a critical set for $\mathscr{F}$ (see for example [28]). But, this result is not true for critical sets for matroids in general. Consider the matroids $(M, N)$ in Remark 4.4.11 and the sequence $\left\langle A_{i}: i \in \omega\right\rangle$ such that $A_{i} \subseteq E$ with $\left|A_{i}\right|=i$ and $A_{\alpha} \subseteq A_{\beta}$ for every $\alpha \leq \beta<\omega$. Observe that $\left\langle A_{i}: i \in \omega\right\rangle$ is a sequence of critical sets for $(M, N)$. But $\bigcup_{\alpha<\omega} A_{a}=E$ which is not a critical set for $(M, N)$ because $(M, N)$ has no covering. 


\section{Chapter 5}

\section{Matroid Intersection Conjecture for}

\section{Singular Matroids}

\subsection{Introduction}

In this chapter assume that $M$ and $N$ are matroids on a common ground set $E$. In Chapter 5, we first introduce the following condition which is equivalent to the condition (X1) introduced in 4.1 .

Definition 5.1.1. We say that $(M, N)$ has a blockage if and only if

- there exists a critical set $K \subseteq E$ for $(M, N)$ and $a \in E \backslash K$ such that $a \in \mathrm{cl}_{M}(K)$ and $a \in \mathrm{cl}_{N}(K)$.

The first main result in this chapter is the following and concerns arbitrary matroids. Recall that we say $(M, N)$ has the Packing/Covering Property if and only if there exists a partition $E=P \sqcup C$ such that $\left(M \uparrow_{P}, N \uparrow_{P}\right)$ has a packing and (M.C,N.C) has a covering.

Theorem 5.1.2. Suppose the followings are equivalent:

1. $(M, N)$ has a covering. 
2. $(M, N)$ has no blockage.

Then $(M, N)$ has the Packing/Covering Property.

The next main results in this chapter are the following and for a particular matroids that we call them singular. We say $M$ and $N$ are singular if and only if they are disjoint union of matroids either uniform of rank one or uniform of co-rank one.

Theorem 5.1.3. If matroids $M$ and $N$ are singular, then there exists a maximal critical set for $M$ and $N$.

Theorem 5.1.4. Let $M$ and $N$ be singular matroids on an infinite countable set $E$. Then the followings are equivalent:

1. $(M, N)$ has a covering.

2. $(M, N)$ has no blockage.

Theorem 5.1.4 and Theorem 5.1.2 imply that the Matroid Intersection Conjecture is true for singular matroids on an infinite countable set.

Corollary 5.1.5. If matroids $M$ and $N$ are singular on an infinite countable set $E$, then $M$ and $N$ satisfy the Matroid Intersection Conjecture.

In section 2, we prove Theorem 5.1.2. The remaining sections cover the proof of Theorem 5.1.3. and Corollary 5.1.4. and the main part of the proof is in Section 4.

\subsection{Proof of Theorem 5.1.2}

Definition 5.2.1. We say $(M, N)$ is loose if and only if for every nonempty set $P \subseteq E$, the pair $\left(M \uparrow_{P}, N \uparrow_{P}\right)$ has no packing.

Lemma 5.2.2. Let $(M, N)$ be any matroids on $E$. There exists a partition $E=P \sqcup C$ such that $\left(M \uparrow_{P}, N \uparrow_{P}\right)$ has a packing and (M.C,N.C) is loose. 
Proof. Let

$$
\mathscr{A}=\left\{(P, \mathscr{P}): P \subseteq E \text { and } \mathscr{P}=\left(S_{1}, S_{2}\right) \text { being a packing for }\left(M \uparrow_{P}, N \uparrow_{P}\right)\right\}
$$

Let $(P, \mathscr{P}),\left(P^{\prime}, \mathscr{P}^{\prime}\right) \in \mathscr{A}$ and define

$$
(P, \mathscr{P}) \leq\left(P^{\prime}, \mathscr{P}^{\prime}\right) \quad \text { if and only if } \quad P \subseteq P^{\prime}, S_{1} \subseteq S_{1}^{\prime}, S_{2} \subseteq S_{2}^{\prime}
$$

Let $\left(A_{\alpha}: \alpha<\gamma\right)$ be a sequence of elements of $\mathscr{A}$ such that $A_{\alpha} \subseteq A_{\beta}$ for $\alpha \leq \beta$. Observe that $\bigcup_{\alpha<\gamma} A_{\alpha} \in \mathscr{A}$. Therefore by Zorn's Lemma, there exists a maximal element $(\bar{P}, \bar{P})$ in $\mathscr{A}$. Let $C=E \backslash \bar{P}$. Then (M.C,N.C) is loose.

Now we are ready to prove the Theorem 5.1.2.

Proof of Theorem 5.1.2. By the lemma 5.2.2, there exists a partition $E=P \sqcup C$ such that $\left(M \uparrow_{P}, N \uparrow_{P}\right)$ has a packing and (M.C,N.C) is loose. If (M.C,N.C) has a covering, then $(M, N)$ has the Packing/Covering Property. Suppose, for a contradiction, that (M.C,N.C) has no covering. Then by the assumption, (M.C,N.C) has a blockage. So, there exists a critical set $K \subseteq C$ for (M.C,N.C) and $a \in C \backslash K$ such that $a \in \mathrm{cl}_{M . C}(K)$ and $a \in \mathrm{cl}_{N . C}(K)$. Let $\left(M^{\prime}, N^{\prime}\right)=(M . C, N . C)$. Since $K$ is a critical set for $\left(M^{\prime}, N^{\prime}\right)$, we have $\left(M^{\prime} \uparrow_{K}, N^{\prime} \uparrow_{K}\right)$ has a covering. Let $(I, J)$ be a covering for $\left(M^{\prime} \uparrow_{K}, N^{\prime} \uparrow_{K}\right)$. Since $K$ is a critical set for $\left(M^{\prime}, N^{\prime}\right)$, we have $(I, J)$ is also a packing for $\left(M^{\prime} \uparrow_{K}, N^{\prime} \uparrow_{K}\right)$. But $\left(M^{\prime}, N^{\prime}\right)$ is loose and this implies that $K=\emptyset$. Since $a \in \mathrm{cl}_{M^{\prime}}(K)$ and $a \in \mathrm{cl}_{N^{\prime}}(K)$ and $K=\emptyset$, we have $\{a\}$ is a loop of $M^{\prime}$ and $N^{\prime}$. This implies that $(\emptyset, \emptyset)$ is a packing for $\left(M^{\prime} \uparrow_{\{a\}}, N^{\prime} \uparrow_{\{a\}}\right)$. So, $\left(M^{\prime} \uparrow_{\{a\}}, N^{\prime} \uparrow_{\{a\}}\right)$ has a packing and this is a contradiction with $\left(M^{\prime}, N^{\prime}\right)$ is loose. 


\subsection{Proof of Theorem 5.1.3}

One of the key elements in our proof for Theorem 5.1.4 is using maximal critical sets. In this section we show that for singular matroids maximal critical sets exists. Here, we first provide an explicit definition of singular matroids.

Definition 5.3.1. Matroids $M$ and $N$ on a common ground set $E$ are called singular if and only if the followings hold:

1. There exists a partition of $E=\bigsqcup_{i \in I} E_{i}$ such that

$$
M=\bigoplus_{i \in I} M_{i}
$$

with each $M_{i}$ being either a uniform matroid of rank one or a uniform matroid co-rank one on the set $E_{i}$.

2. There exists a partition of $E=\bigsqcup_{j \in J} E_{j}^{\prime}$ such that

$$
N=\bigoplus_{j \in J} N_{j}
$$

with each $N_{j}$ being either a uniform matroid of rank one or a uniform matroid co-rank one on the set $E_{j}^{\prime}$.

First, observe that for singular matroids $M$ and $N$ where each $M_{i}$ and each $N_{j}$ being a uniform matroid of rank one, we have $M$ and $N$ are partition matroids. So, the family of singular matroids contains the family of partition matroids. Also, for singular matroids $M$ and $N$ where each $M_{i}$ is a uniform matroid of co-rank 1 and each $N_{j}$ is a uniform matroid of rank one, we have $M^{*}$ and $N$ are partition matroids corresponding to a bipartite graph with the edges sets $E$.

Lemma 5.3.2. Let $(M, N)$ be singular, $K \subseteq E$ a critical set for $(M, N)$, and $L \subseteq E \backslash K$ a critical set for $(M / K, N / K)$. Then $K \cup L$ is a critical set for $(M, N)$. 


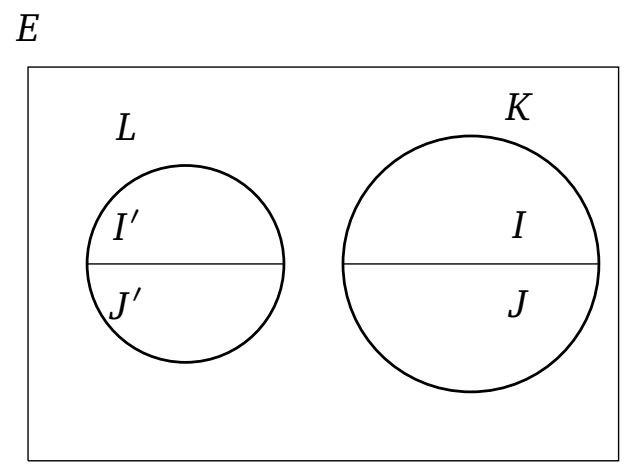

Figure 5.3.1: The covering $(I, J)$ for $\left(M \uparrow_{K}, N \uparrow_{K}\right)$ and the covering $\left(I^{\prime}, J^{\prime}\right)$ for $\left(M^{\prime} \uparrow_{L}, N^{\prime} \uparrow_{L}\right)$.

Proof. Since $K$ is a critical set for $(M, N)$, there exists a covering $(I, J)$ for $\left(M \uparrow_{K}, N \uparrow_{K}\right)$. Let $\left(M^{\prime}, N^{\prime}\right)=(M / K, N / K)$. Since $L$ is a critical set for $\left(M^{\prime}, N^{\prime}\right)$, there exists a covering $\left(I^{\prime}, J^{\prime}\right)$ for $\left(M^{\prime} \uparrow_{L}, N^{\prime} \uparrow_{L}\right)$. See the figure 5.3.1. Since $I^{\prime}$ is independent in $M^{\prime}=M / K$, we have $K \cup I^{\prime}$ is independent in $M$, hence $I \cup I^{\prime}$ is independent in $M$. Similarly, $J \cup J^{\prime}$ is independent in $N$. Thus $\left(I \cup I^{\prime}, J \cup J^{\prime}\right)$ is a covering for $\left(M \uparrow_{(K \cup L)}, N \uparrow_{(K \cup L)}\right)$. To show that $K \cup L$ is a critical set for $(M, N)$, it remains to show that any covering $\left(I^{\prime \prime}, J^{\prime \prime}\right)$ for $\left(M \uparrow_{(K \cup L)}, N \uparrow_{(K \cup L)}\right)$ is also a packing. Since $K$ is critical for $(M, N)$ and $\left(I^{\prime \prime} \cap K, J^{\prime \prime} \cap K\right)$ is a covering for $\left(M \uparrow_{K}, N \uparrow_{K}\right)$, the set $I^{\prime \prime} \cap K$ spans $K$ in $M$ and the set $J^{\prime \prime} \cap K$ spans $K$ in $N$. So, it remains to show that $I^{\prime \prime}$ spans $L$ in $M$ and $J^{\prime \prime}$ spans $L$ in $N$. Now, consider $\left(I^{\prime \prime} \cap L, J^{\prime \prime} \cap L\right)$ which is a covering for $\left(M \uparrow_{L}, N \uparrow_{L}\right)$.

First, we show that $\left(I^{\prime \prime} \cap L, J^{\prime \prime} \cap L\right)$ is also a covering for $\left(M^{\prime} \uparrow_{L}, N^{\prime} \uparrow_{L}\right)$. It is enough to show that $I^{\prime \prime} \cap L$ is independent in $M^{\prime}$ and $J^{\prime \prime} \cap L$ is independent in $N^{\prime}$. Suppose, for a contradiction, that there exists a circuit $C$ of $M^{\prime}$ such that $C \subseteq I^{\prime \prime} \cap L$. Since $I^{\prime \prime} \cap L$ is independent in $M$, the set $C$ is independent in $M$ and can be extended to $C^{M}$ a circuit of $M$ such that

$$
C^{M} \subseteq\left(I^{\prime \prime} \cap L\right) \cup K
$$

Since $(M, N)$ is singular, $M=\bigoplus_{r \in R} M_{r}$ with each $M_{r}$ being either a uniform matroid of rank one or a uniform matroid of co-rank one on the set $E_{r}$. So, there exists some $r \in R$ such that $C^{M}$ is a circuit of the matroid $M_{r}$. Now, we have two possibilities: either $M_{r}$ is a uniform matroid of 
rank one, or $M_{r}$ is a uniform matroid of co-rank one.

If $M_{r}$ is a uniform matroid of rank one, then the circuit $C^{M}=\{a, b\}$ with $a \in I^{\prime \prime} \cap L$ and $b \in K$. Observe that since $I^{\prime \prime}$ is independent in $M$, we have $b \in J^{\prime \prime} \cap K$. Since $I^{\prime \prime} \cap K$ spans $K$ in $M$, it spans $\{b\}$ in $M$. Thus, there is a circuit $C_{b}$ of $M$ such that

$$
b \in C_{b} \subseteq\left(I^{\prime \prime} \cap K\right) \cup\{b\} .
$$

Observe that $C_{b}$ is also a circuit of the matroid $M_{r}$. Therefor, $C_{b}=\{b, c\}$ with $c \in\left(I^{\prime \prime} \cap K\right)$. Now by the circuit elimination axiom and eliminating $\{b\}$ from the circuits $C^{M}$ and $C_{b}$, there is a circuit $C^{\prime}$ of $M_{r}$ such that $C^{\prime} \subseteq\{a, c\}$. Since $M_{r}$ is a uniform matroid of rank one, the set $\{a, c\}$ is the circuit $C^{\prime}$ of $M_{r}$. But, $\{a, c\} \subseteq I^{\prime \prime}$ which is a contradiction with $I^{\prime \prime}$ is independent in $M$.

If $M_{r}$ is a uniform matroid of co-rank one, then $C^{M}=E_{r}$. Since $I^{\prime \prime}$ is independent in $M$, we have $C^{M} \cap J^{\prime \prime} \cap K \neq \emptyset$. Let

$$
x \in C^{M} \cap J^{\prime \prime} \cap K .
$$

Since $I^{\prime \prime} \cap K$ spans $K$ in $M$, there is a circuit $C_{x}$ of $M$ such that

$$
x \in C_{x} \subseteq\left(I^{\prime \prime} \cap K\right) \cup\{x\} .
$$

Since $x \in E_{r}$ and $M_{r}$ is a uniform matroid of co-rank one, $C_{x}=E_{r}$. This implies that $C^{M}=C_{x}$, but $C_{x} \subseteq K$ and hence $C_{x} \cap L=\emptyset$. This is a contradiction because $C^{M} \cap L \neq \emptyset$.

So, we proved that $I^{\prime \prime} \cap L$ is independent in $M^{\prime}$. Similarly, it can be shown that $J^{\prime \prime} \cap L$ is independent in $N^{\prime}$. Thus, $\left(I^{\prime \prime} \cap L, J^{\prime \prime} \cap L\right)$ is a covering for $\left(M^{\prime} \uparrow_{L}, N^{\prime} \uparrow_{L}\right)$. Since $L$ is a critical set for $\left(M^{\prime}, N^{\prime}\right)$, the covering $\left(I^{\prime \prime} \cap L, J^{\prime \prime} \cap L\right)$ is also a packing for $\left(M^{\prime} \uparrow_{L}, N^{\prime} \uparrow_{L}\right)$. Hence, $I^{\prime \prime} \cap L$ spans $L$ in $M^{\prime}$. Let $y \in\left(J^{\prime \prime} \cap L\right)$. There exists a circuit $C_{y}$ of $M^{\prime}$ such that

$$
y \in C_{y} \subseteq I^{\prime \prime} \cap L .
$$


If $C_{y}$ is a circuit of $M$, then $\{y\}$ is spanned in $M$ by $I^{\prime \prime} \cap L$. Otherwise, the set $C_{y}$ can be extended to $C_{y}^{M}$ a circuit of $M$ such that

$$
y \in C_{y} \subseteq C_{y}^{M} \subseteq\left(I^{\prime \prime} \cap L\right) \cup K \cup\{y\} .
$$

Let $s \in R$ be such that the circuit $C_{y}^{M} \subseteq E_{s}$.

First assume $M_{s}$ is a uniform matroid of rank one. If $E_{s} \cap I^{\prime \prime} \neq \emptyset$, then $\{y\}$ is spanned in $M$ by $I^{\prime \prime}$. Otherwise, $E_{s} \cap I^{\prime \prime}=\emptyset$ and hence $C_{y}^{M} \cap J^{\prime \prime} \cap K \neq \emptyset$. Let

$$
z \in C_{y}^{M} \cap J^{\prime \prime} \cap K
$$

Since $K$ is critical for $(M, N)$, the set $\{z\}$ is spanned in $M$ by $I^{\prime \prime} \cap K$. Thus there is a circuit $C_{z}$ of $M$ such that

$$
z \in C_{z} \subseteq\left(I^{\prime \prime} \cap K\right) \cup\{z\}
$$

Then $C_{z} \cap I^{\prime \prime} \subseteq E_{s}$ which is a contradiction with $E_{s} \cap I^{\prime \prime}=\emptyset$.

Now, assume that $M_{s}$ is a uniform matroid of co-rank one. Then $C_{y}^{M}=E_{s}$. If

$$
C_{y}^{M} \subseteq I^{\prime \prime} \cup\{y\}
$$

then $\{y\}$ is spanned in $M$ by $I^{\prime \prime}$. Otherwise, $C_{y}^{M} \cap J^{\prime \prime} \cap K \neq \emptyset$. Let

$$
w \in C_{y}^{M} \cap J^{\prime \prime} \cap K .
$$

Since $K$ is critical for $(M, N)$, the set $\{w\}$ is spanned in $M$ by $I^{\prime \prime} \cap K$. Therefore, there exists a circuit $C_{w}$ of $M$ such that

$$
w \in C_{w} \subseteq\left(I^{\prime \prime} \cap K\right) \cup\{w\}
$$

Since $M_{s}$ is a uniform matroid of co-rank one and $w \in E_{s}$, the circuit $C_{w}=E_{s}$. Hence $C_{y}^{M}=C_{w}$. But since $C_{w} \subseteq K$, we have $C_{w} \cap L=\emptyset$ and this is a contradiction with $C_{y}^{M} \cap L \neq \emptyset$. 
Thus, we proved that for any $y \in J^{\prime \prime} \cap L$, the set $\{y\}$ is spanned in $M$ by $I^{\prime \prime}$. This implies that $I^{\prime \prime}$ is a spanning set for the matroid $M \uparrow_{(K \cup L)}$. By a similar argument, it can be shown that $J^{\prime \prime}$ is a spanning set for the matroid $N \uparrow_{(K \cup L)}$. Therefore, $\left(I^{\prime \prime}, J^{\prime \prime}\right)$ is a packing for $\left(M \uparrow_{(K \cup L)}, N \uparrow_{(K \cup L)}\right)$. This completes the proof that $K \cup L$ is a critical set for $(M, N)$.

Lemma 5.3.3. Let $(M, N)$ be such that it has a special covering. Let $A \subseteq E$ be such that for any covering $(I, J)$ of $(M, N), A \subseteq \mathrm{cl}_{N}(J)$. Then there exists a critical set $K \subseteq E$ for $(M, N)$ such that $A \subseteq K$.

Proof. We want to show that any $a \in A$ is essential for $(M, N)$. Suppose, for a contradiction, that there is some $a \in A$ that is not essential for $(M, N)$. Therefore, $(M /\{a\}, N /\{a\})$ has a covering. Let $(I, J)$ be a covering for $(M /\{a\}, N /\{a\})$. Then $(I \cup\{a\}, J)$ is a covering for $(M, N)$ and $a \notin \operatorname{cl}_{N}(J)$ which is a contradiction with $a \in A \subseteq \operatorname{cl}_{N}(J)$. So, for any $a \in A$, $a$ is essential for $(M, N)$. Since $(M, N)$ has a special covering, by lemma 4.1.11 there exist a critical set $K \subseteq E$ for $(M, N)$ such that $A \subseteq K$.

By a similar argument we can show the following result.

Lemma 5.3.4. Let $(M, N)$ be such that it has a special covering. Let $A \subseteq E$ be such that for any covering $(I, J)$ of $(M, N), A \subseteq \mathrm{cl}_{M}(I)$. Then there exists a critical set $K \subseteq E$ for $(M, N)$ such that $A \subseteq K$.

Now we are ready to prove Theorem 5.1.3.

Proof of Theorem 5.1.3. If $(M, N)$ has a covering, then by the lemma 4.4.10, there exists a maximal critical set. Otherwise, let $E^{\prime} \subseteq E$ be a maximal subset of $E$ such that $\left(M \uparrow_{E^{\prime}}, N \uparrow_{E^{\prime}}\right)$ has a covering. Let $(I, J)$ be a covering for $\left(M \uparrow_{E^{\prime}}, N \uparrow_{E^{\prime}}\right)$ and $a \in E \backslash E^{\prime}$. Since $E^{\prime}$ is maximal, $\left(M \uparrow_{E^{\prime \prime}}, N \uparrow_{E^{\prime \prime}}\right)$ has no covering in which $E^{\prime \prime}=E^{\prime} \cup\{a\}$. Therefore,

$$
a \in \operatorname{cl}_{M}(I) \text { and } a \in \operatorname{cl}_{N}(J)
$$




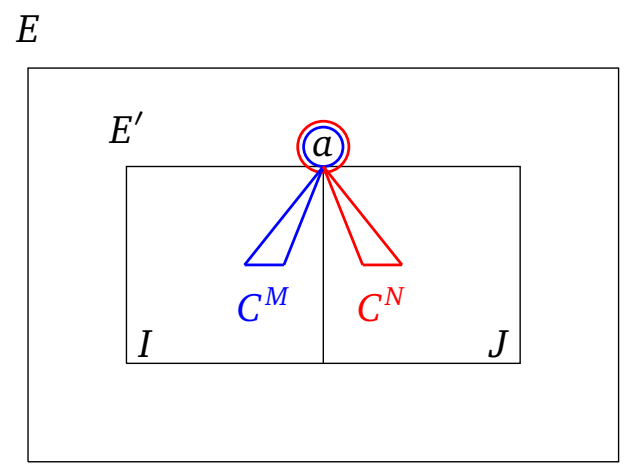

Figure 5.3.2: The circuits $C^{M}$ and $C^{N}$.

Let $C^{M}$ be the circuit of $M$ such that

$$
a \in C^{M} \subseteq I \cup\{a\}
$$

and $C^{N}$ be the circuit of $N$ such that

$$
a \in C^{N} \subseteq J \cup\{a\}
$$

See the figure 5.3.2. We first show that there exists a critical set $K_{1} \subseteq E^{\prime}$ for $(M, N)$ such that

$$
C^{M} \backslash\{a\} \subseteq K_{1}
$$

Since $(M, N)$ is singular, $M=\bigoplus_{r \in R} M_{r}$ with each $M_{r}$ being either a uniform matroid of rank one or uniform matroid of co-rank one on the set $E_{r}$. Let $r \in R$ be such that $C^{M} \subseteq E_{r}$.

If $M_{r}$ is a uniform matroid of rank one, then $C^{M}=\{a, x\}$ with $x \in I$. We want to show that for any covering $\left(I^{\prime}, J^{\prime}\right)$ for $\left(M \uparrow_{E^{\prime}}, N \uparrow_{E^{\prime}}\right)$, we have $x \in \mathrm{cl}_{N}\left(J^{\prime}\right)$. Suppose, for a contradiction, that there exists a covering $\left(I^{\prime}, J^{\prime}\right)$ for $\left(M \uparrow_{E^{\prime}}, N \uparrow_{E^{\prime}}\right)$ such that $x \notin \mathrm{cl}_{N}\left(J^{\prime}\right)$. This implies that $x \in I^{\prime}$ and $J^{\prime} \cup\{x\}$ is independent in $N$. Since $x \in I^{\prime}$ and $I^{\prime}$ is independent in $M$, we have $E_{r} \cap I^{\prime}=\emptyset$. 
Thus, $I^{\prime} \cup\{a\} \backslash\{x\}$ is independent in $M$. Therefore

$$
\left(I^{\prime} \cup\{a\} \backslash\{x\}, J^{\prime} \cup\{x\}\right)
$$

is a covering for $\left(M \uparrow_{E^{\prime \prime}}, N \uparrow_{E^{\prime \prime}}\right)$. This is a contradiction with $E^{\prime}$ being a maximal set such that $\left(M \uparrow_{E^{\prime}}, N \uparrow_{E^{\prime}}\right)$ has a covering.

If $M_{r}$ is a uniform matroid of co-rank one, then $C^{M}=E_{r}$. We want to show that for any covering $\left(I^{\prime}, J^{\prime}\right)$ for $\left(M \uparrow_{E^{\prime}}, N \uparrow_{E^{\prime}}\right)$, we have

$$
C^{M} \backslash\{a\} \subseteq \operatorname{cl}_{N}\left(J^{\prime}\right)
$$

Suppose, for a contradiction, that there exists a covering $\left(I^{\prime}, J^{\prime}\right)$ for $\left(M \uparrow_{E^{\prime}}, N \uparrow_{E^{\prime}}\right)$ and some

$$
y \in C^{M} \backslash\{a\}
$$

such that $\mathrm{y} \notin \mathrm{cl}_{N}\left(J^{\prime}\right)$. So, $J^{\prime} \cup\{y\}$ is independent in $N$. Observe that $I^{\prime} \cup\{a\} \backslash\{y\}$ is independent in $M$ because if there is a circuit $C$ of $M$ such that $C \subseteq I^{\prime} \cup\{a\} \backslash\{y\}$, then sine $a \in C$ and $M_{r}$ is a uniform matroid of co-rank one, we have $C=E_{r}$. Hence $C^{M}=C$, but $y \in C^{M} \backslash C$ which is a contradiction. Therefore,

$$
\left(I^{\prime} \cup\{a\} \backslash\{y\}, J^{\prime} \cup\{y\}\right)
$$

is a covering for $\left(M \uparrow_{E^{\prime \prime}}, N \uparrow_{E^{\prime \prime}}\right)$. This is a contradiction with $E^{\prime}$ being a maximal set such that $\left(M \uparrow_{E^{\prime}}, N \uparrow_{E^{\prime}}\right)$ has a covering.

Thus, we proved that for any covering $\left(I^{\prime}, J^{\prime}\right)$ of $\left(M \uparrow_{E^{\prime}}, N \uparrow_{E^{\prime}}\right)$, we have $C^{M} \backslash\{a\} \subseteq \mathrm{cl}_{N}\left(J^{\prime}\right)$. Now we can apply lemma 5.3.3 for the matroids $\left(M \uparrow_{E^{\prime}}, N \uparrow_{E^{\prime}}\right)$. Since $\left(M \uparrow_{E^{\prime}}, N \uparrow_{E^{\prime}}\right)$ has a covering and is singular, it has a special covering. Thus, by the lemma 5.3.3 there exists a critical set $K_{1} \subseteq E^{\prime}$ for $\left(M \uparrow_{E^{\prime}}, N \uparrow_{E^{\prime}}\right)$ such that $C^{M} \backslash\{a\} \subseteq K_{1}$. By a similar argument, we can show that exists a critical set $K_{2} \subseteq E^{\prime}$ for $\left(M \uparrow_{E^{\prime}}, N \uparrow_{E^{\prime}}\right)$ such that $C^{N} \backslash\{a\} \subseteq K_{2}$.

Since $\left(M \uparrow_{E^{\prime}}, N \uparrow_{E^{\prime}}\right)$ has a covering and $K_{1}$ and $K_{2}$ are critical sets for $\left(M \uparrow_{E^{\prime}}, N \uparrow_{E^{\prime}}\right)$ by the 
lemma 4.4.10, we have $K=K_{1} \cup K_{2}$ is a critical set for $\left(M \uparrow_{E^{\prime}}, N \uparrow_{E^{\prime}}\right)$, such that

$$
\left(C^{M} \cup C^{N}\right) \backslash\{a\} \subseteq K
$$

Therefore, we proved that for any $a \in E \backslash E^{\prime}$, there exists a critical set $K \subseteq E^{\prime}$ for $\left(M \uparrow_{E^{\prime}}, N \uparrow_{E^{\prime}}\right)$ such that $\left(C^{M} \cup C^{N}\right) \backslash\{a\} \subseteq K$ where $C^{M}$ and $C^{N}$ are the unique circuits of $M$ and $N$ with $a \in C^{M} \subseteq I \cup\{a\}$ and $a \in C^{N} \subseteq J \cup\{a\}$.

Now, let

$$
\mathscr{A}=\left\{K \subseteq E^{\prime}: K \text { is a critical set for }(M, N)\right\} \text {. }
$$

Since $\left(M \uparrow_{E^{\prime}}, N \uparrow_{E^{\prime}}\right)$ has a covering by the lemma 4.4.10, we have $\bigcup \mathscr{A}$ is a critical set for $(M, N)$. We want to show that $\bigcup \mathscr{A}$ is a maximal critical set for $(M, N)$. Suppose, for a contradiction, that $\bigcup \mathscr{A}$ is not maximal and it can be extended to a critical set $\bar{K}$ for $(M, N)$ with

$$
\bigcup \mathscr{A} \subseteq \bar{K}
$$

Observe that $\bar{K} \backslash E^{\prime} \neq \emptyset$. Let $a \in \bar{K} \backslash E^{\prime}$ and $C^{M}$ be the unique circuit of $M$ such that

$$
a \in C^{M} \subseteq I \cup\{a\}
$$

and $C^{N}$ the unique circuit of $N$ such that

$$
a \in C^{N} \subseteq J \cup\{a\}
$$

Let $(\bar{I}, \bar{J})$ be a covering for $\left(M \uparrow_{\bar{K}}, N \uparrow_{\bar{K}}\right)$. Since $a \in \bar{K} \backslash E^{\prime}$, there exists a critical set $K \subseteq E^{\prime}$ for $\left(M \uparrow_{E^{\prime}}, N \uparrow_{E^{\prime}}\right)$ such that $\left(C^{M} \cup C^{N}\right) \backslash\{a\} \subseteq K$. Since $K \subseteq E^{\prime}$ is a critical set for $\left(M \uparrow_{E^{\prime}}, N \uparrow_{E^{\prime}}\right)$, we have $K \subseteq \bigcup \mathscr{A}$. This implies that $K \subseteq \bar{K}$ and in particular

$$
C^{M} \cup C^{N} \subseteq \bar{K}
$$


Observe that $(\bar{I} \cap K, \bar{J} \cap K)$ is a covering for $\left(M \uparrow_{K}, N \uparrow_{K}\right)$. Since $K$ is a critical set for $\left(M \uparrow_{E^{\prime}}, N \uparrow_{E^{\prime}}\right)$, the covering $(\bar{I} \cap K, \bar{J} \cap K)$ is also a packing.

If $a \in \bar{I}$, then $C^{M} \nsubseteq \bar{I}$ because $\bar{I}$ is independent in $M$. Let $z \in C^{M} \backslash \bar{I}$. Since $z \in \bar{J} \cap K$ and $(\bar{I} \cap K, \bar{J} \cap K)$ is a packing, we have $z \in \operatorname{cl}_{M}(\bar{I} \cap K)$. Thus, there exists a circuit $C_{z}$ of $M$ such that

$$
z \in C_{z} \subseteq(\bar{I} \cap K) \cup\{z\}
$$

Let $s \in R$ be such that $z \in E_{s}$.

If $M_{s}$ is a uniform matroid of rank one, then $C^{M}=\{a, z\}$ and $C_{z}=\{z, w\}$ for some $w \in \bar{I}$. By the circuit elimination axiom and eliminating $z$ from the circuits $C^{M}$ and $C_{z}$ we get $\{a, w\}$ is a circuit of $M$. But, $\{a, w\} \subseteq \bar{I}$ which is a contradiction with $\bar{I}$ is independent in $M$. If $M_{s}$ is a uniform matroid of co-rank one, then $C^{M}=E_{s}=C_{z}$ but $a \in C^{M} \backslash C_{z}$ which is a contradiction.

If $a \in \bar{J}$, then $C^{N} \nsubseteq \bar{J}$ because $\bar{J}$ is independent in $N$. Then, by a similar argument we get a contradiction. This completes the proof that $\bigcup \mathscr{A}$ is a maximal critical set for $(M, N)$.

\subsection{Proof of Theorem 5.1.4 and Corollary 5.1.5}

In this section, we first provide some required lemma for our proof of Theorem 5.1.4 and then we prove Theorem 5.1.4.

Lemma 5.4.1. Let $(M, N)$ be singular, $e \in E$, and $K$ a maximal critical set for $(M \backslash\{e\}, N \backslash\{e\})$ such that $e \notin \mathrm{cl}_{N}(K)$. Then, there exists no critical set $K^{*}$ for $(M \backslash\{e\}, N \backslash\{e\})$ such that $e \in \mathrm{cl}_{N}\left(K^{*}\right)$.

Proof. Suppose, for a contradiction, that there is a critical set $K^{*}$ for $(M \backslash\{e\}, N \backslash\{e\})$ such that $e \in \mathrm{cl}_{N}\left(K^{*}\right)$.

First observe that $K^{*} \backslash \mathrm{cl}_{N}(K) \neq \emptyset$. Otherwise, if $K^{*} \subseteq \mathrm{cl}_{N}(K)$, then

$$
\mathrm{cl}_{N}\left(K^{*}\right) \subseteq \mathrm{cl}_{N}\left(\mathrm{cl}_{N}(K)\right)=\mathrm{cl}_{N}(K)
$$




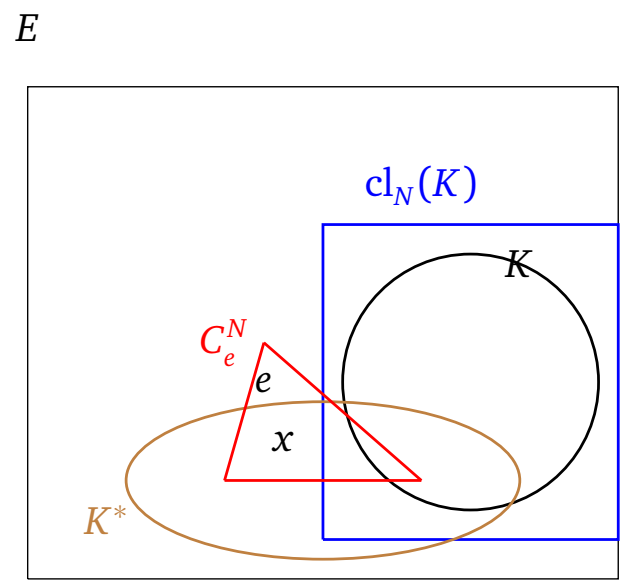

Figure 5.4.1: Critical sets $K$ and $K^{*}$ and the circuit $C_{e}^{N}$.

But $e \in \mathrm{cl}_{N}\left(K^{*}\right)$ and this implies that $e \in \mathrm{cl}_{N}(K)$ which is a contradiction with the assumption. Therefore, $K^{*} \backslash \operatorname{cl}_{N}(K) \neq \emptyset$. Since $e \in \mathrm{cl}_{N}\left(K^{*}\right)$, there is a circuit $C_{e}^{N}$ of $N$ such that

$$
e \in C_{e}^{N} \subseteq\left(K^{*} \cup\{e\}\right)
$$

Observe that $C_{e}^{N} \backslash\left(\mathrm{cl}_{N}(K) \cup\{e\}\right) \neq \emptyset$ because otherwise $e \in \mathrm{cl}_{N}(K)$ which is a contradiction with the assumption. Let $x \in C_{e}^{N} \backslash\left(\mathrm{cl}_{N}(K) \cup\{e\}\right)$. See the figure 5.4.1. We want to show that $x \notin \mathrm{cl}_{M}(K)$.

Suppose, for a contradiction, that $x \in \operatorname{cl}_{M}(K)$. We get a contradiction by showing that $K \cup\{x\}$ is a critical set for $(M, N)$. Since $K$ is a critical set for $(M, N)$, for any covering $(I, J)$ for $\left(M \uparrow_{K}, N \uparrow_{K}\right)$, we have $x \in \operatorname{cl}_{M}(I)$. Since $x \notin K$, this implies that for any covering $(I, J)$ for $\left(M \uparrow_{K}, N \uparrow_{K}\right)$, we have $I \cup\{x\}$ is dependent in $M$. Since $x \notin \mathrm{cl}_{N}(K)$, we have $K \cup\{x\}$ is independent in $N$. Thus, for any covering $(I, J)$ for $\left(M \uparrow_{K}, N \uparrow_{K}\right)$, we have $J \cup\{x\}$ is independent in $N$. Therefore, $(I, J \cup\{x\})$ is a covering for $\left(M \uparrow_{(K \cup\{x\})}, N \uparrow_{(K \cup\{x\})}\right)$. Now, we want to show that any covering $\left(I^{\prime}, J^{\prime}\right)$ for $\left(M \uparrow_{(K \cup\{x\})}, N \uparrow_{(K \cup\{x\})}\right)$ is also a packing. First observe that $\left(I^{\prime}, J^{\prime} \backslash\{x\}\right)$ is a covering for $\left(M \uparrow_{K}, N \uparrow_{K}\right)$. Since $K$ is a critical set for $(M, N),\left(I^{\prime}, J^{\prime} \backslash\{x\}\right)$ is also a packing for $\left(M \uparrow_{K}, N \uparrow_{K}\right)$. Therefore, $I^{\prime}$ is spanned in $N$ by $J^{\prime} \backslash\{x\}$ and $J^{\prime} \backslash\{x\}$ is spanned in $M$ by $I^{\prime}$. So, to show that $\left(I^{\prime}, J^{\prime} \backslash\{x\}\right)$ is a packing, it remains to show that $\{x\}$ is spanned in $M$ by 


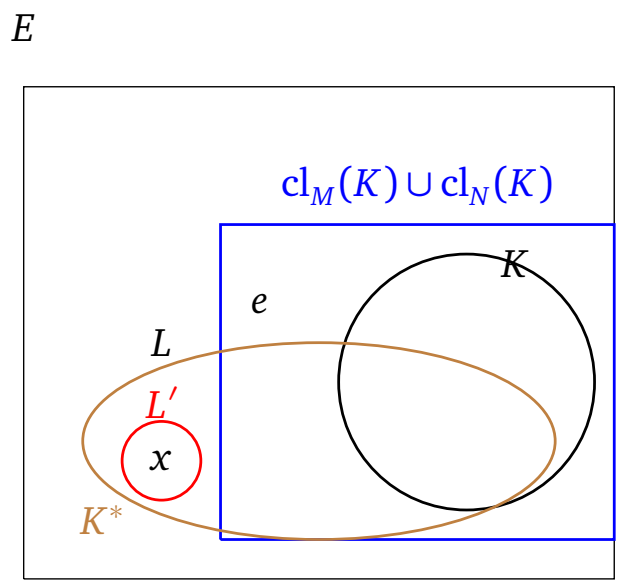

Figure 5.4.2: The sets $L$ and $L^{\prime}$.

$I^{\prime}$. This is true because $I^{\prime} \cup\{x\}$ is dependent in $M$. This completes the proof that $K \cup\{x\}$ is a critical set for $(M, N)$. As $e \notin(K \cup\{x\})$, we have $K \cup\{x\}$ is a critical set for $(M \backslash\{e\}, N \backslash\{e\})$. Now, this is a contradiction with $K$ being a maximal critical set for $(M \backslash\{e\}, N \backslash\{e\})$.

Since $x \in K^{*}$ and $x \notin \mathrm{cl}_{N}(K)$ and we proved that $x \notin \mathrm{cl}_{M}(K)$, we have

$$
K^{*} \backslash\left(\mathrm{cl}_{M}(K) \cup \mathrm{cl}_{N}(K)\right) \neq \emptyset \text {. }
$$

Let $L=K^{*} \backslash\left(\operatorname{cl}_{M}(K) \cup \mathrm{cl}_{N}(K)\right)$. We want to prove that there exists a critical set $L^{\prime} \subseteq L$ for $(M / K, N / K)$. Let $\left(M^{\prime}, N^{\prime}\right)=(M / K, N / K)$.

Let $(I, J)$ be a covering for $\left(M \uparrow_{K^{*}}, N \uparrow_{K^{*}}\right)$. If

$$
\left(I \backslash\left(\mathrm{cl}_{M}(K) \cup \mathrm{cl}_{N}(K)\right), J \backslash\left(\mathrm{cl}_{M}(K) \cup \mathrm{cl}_{N}(K)\right)\right)
$$

are independent in $\left(M^{\prime}, N^{\prime}\right)$, then it is a covering for $\left(M^{\prime} \uparrow_{K}, N^{\prime} \uparrow_{K}\right)$ and we let $L^{\prime}=L$. Otherwise, either $I \backslash\left(\mathrm{cl}_{M}(K) \cup \mathrm{cl}_{N}(K)\right)$ is dependent in $M^{\prime}$ or $J \backslash\left(\mathrm{cl}_{M}(K) \cup \mathrm{cl}_{N}(K)\right)$ is dependent in $N^{\prime}$. If $I \backslash\left(\mathrm{cl}_{M}(K) \cup \mathrm{cl}_{N}(K)\right)$ is dependent in $M^{\prime}$, then there exists a circuit $C^{M^{\prime}}$ of $M^{\prime}$ such that

$$
C^{M^{\prime}} \subseteq I \backslash\left(\mathrm{cl}_{M}(K) \cup \mathrm{cl}_{N}(K)\right)
$$


Since $I$ is independent in $M$, the set $C^{M^{\prime}}$ can be extended to a circuit $C^{M}$ of $M$ such that $C^{M} \backslash C^{M^{\prime}} \subseteq K$. Since $(M, N)$ is singular, $M=\bigoplus_{r \in R} M_{r}$ with each $M_{r}$ being either a uniform matroid of rank one or a uniform matroid of co-rank one on the set $E_{r}$. Let $r \in R$ be such that $C^{M} \subseteq E_{r}$. We first show that $M_{r}$ can not be a uniform matroid of rank one. This is true because if $M_{r}$ is a uniform matroid of rank one, then $C^{M}=\{a, b\}$ such that $a \in L$ and $b \in K$. Since $b \in K$, and $\{a, b\}$ is a circuit of $M$, we have $a \in \operatorname{cl}_{M}(K)$ which is a contradiction with $a \in L$. This implies that $M_{r}$ is a uniform matroid of co-rank 1 and $C^{M}=E_{r}$. Let

$$
\mathscr{C}=\left\{C^{M^{\prime}} \subseteq I \backslash\left(\operatorname{cl}_{M}(K) \cup \mathrm{cl}_{N}(K)\right): C^{M^{\prime}} \text { is a circuit of } M^{\prime}\right\}
$$

If $J \backslash\left(\operatorname{cl}_{M}(K) \cup \mathrm{cl}_{N}(K)\right)$ is dependent in $N^{\prime}$, by a similar argument we can show that any circuit $C^{N^{\prime}}$ of $N^{\prime}$ such that $C^{N^{\prime}} \subseteq I \backslash\left(\operatorname{cl}_{M}(K) \cup \mathrm{cl}_{N}(K)\right)$ does not belong to a uniform matroid of rank one. Let

$$
\mathscr{D}=\left\{C^{N^{\prime}} \subseteq I \backslash\left(\mathrm{cl}_{M}(K) \cup \mathrm{cl}_{N}(K)\right): C^{N^{\prime}} \text { is a circuit of } N^{\prime}\right\}
$$

Therefore

$$
(I \cap L) \backslash \bigcup \mathscr{C}
$$

is independent in $M^{\prime}$ and

$$
(J \cap L) \backslash \bigcup \mathscr{D}
$$

is independent in $N$. Let

$$
L^{\prime}=L \backslash \bigcup(\mathscr{C} \cup \mathscr{D})
$$

We first show that $x \in L^{\prime}$ which implies that $L^{\prime} \neq \emptyset$. Since $e \in C_{e}^{N} \subseteq K^{*} \cup\{e\}$ and $K^{*}$ is a critical set for $(M, N)$ and $(I, J)$ is a covering for $\left(M \uparrow_{K^{*}}, N \uparrow_{K^{*}}\right)$, we have $C_{e}^{N} \backslash\{e\} \subseteq J$. Since $x \in C_{e}^{N}$, we have $x \in J$. Since $x \in L$, if $x \notin L^{\prime}$, then $x \in C^{N^{\prime}}$ such that $C^{N^{\prime}}$ is a circuit of $N^{\prime}$ and it can be extended to $C \subseteq K \cup C^{N^{\prime}}$ a circuit of a uniform matroid of co-rank one. Let $s \in R$ such that $C=E_{s}$. Since $C_{e}^{N}=E_{s}$, we get $C_{e}^{N}=C$, but $e \in C_{e}^{N} \backslash C$ which is a contradiction. Therefore $x \in L^{\prime}$ and hence $L^{\prime} \neq \emptyset$ and $\left(I \cap L^{\prime}, J \cap L^{\prime}\right)$ is a covering for $\left(M^{\prime} \uparrow_{L^{\prime}}, N^{\prime} \uparrow_{L^{\prime}}\right)$. To show that $L^{\prime}$ is 
a critical set for $\left(M^{\prime}, N^{\prime}\right)$, it remains to show that any covering $\left(I^{\prime}, J^{\prime}\right)$ for $\left(M^{\prime} \uparrow_{L^{\prime}}, N^{\prime} \uparrow_{L^{\prime}}\right)$ is also a packing.

Let $\left(I^{\prime}, J^{\prime}\right)$ be a covering for $\left(M^{\prime} \uparrow_{L^{\prime}}, N^{\prime} \uparrow_{L^{\prime}}\right)$. We want to show that $I^{\prime} \cup\left(I \backslash L^{\prime}\right)$ is independent in $M$ and $J^{\prime} \cup\left(J \backslash L^{\prime}\right)$ is independent in $N$, and hence

$$
\left(I^{\prime} \cup\left(I \backslash L^{\prime}\right), J^{\prime} \cup\left(J \backslash L^{\prime}\right)\right)
$$

is a covering for $\left(M \uparrow_{K^{*}}, N \uparrow_{K^{*}}\right)$. Suppose, for a contradiction, that $I^{\prime} \cup\left(I \backslash L^{\prime}\right)$ is dependent in $M$. Then, there exists a circuit $\hat{C}$ of $M$ such that $\hat{C} \subseteq I^{\prime} \cup\left(I \backslash L^{\prime}\right)$. Since $I \backslash L^{\prime}$ is independent in $M$, we have $\hat{C} \cap I^{\prime} \neq \emptyset$. Let $w \in R$ be such that $\hat{C} \subseteq E_{w}$.

If $M_{w}$ is a unifrom matroid of rank one, then $\hat{C}=\{c, d\}$ with $c \in I^{\prime}$ and $d \in I \backslash L^{\prime}$. Observe that $\hat{C} \nsubseteq L$ because otherwise $\hat{C} \subseteq L^{\prime}$ and hence $\hat{C} \subseteq I^{\prime}$ which is a contradiction with $I^{\prime}$ is independent in $M$. Thus, $\hat{C} \backslash L \neq \emptyset$ and $d \in \hat{C} \backslash L$. Therefore $d \in \mathrm{cl}_{M}(K) \cup \mathrm{cl}_{N}(K)$. Observe that $d \notin K$ because otherwise $c \in \operatorname{cl}_{M}(K)$ which is a contradiction with $c \in I^{\prime}$. Since $I^{\prime}$ is independent in $M^{\prime}=M / K$, we have $E_{w} \cap K=\emptyset$. Thus $d \notin \mathrm{cl}_{M}(K)$ and so $d \in \mathrm{cl}_{M}(K) \cup \operatorname{cl}_{N}(K)$ implies that $d \in \operatorname{cl}_{N}(K)$. Now, we show that $K \cup\{d\}$ is a critical set for $(M, N)$. Since $K \cup\{d\}$ is independent in $M$, we have $\left(M \uparrow_{(K \cup\{d\})}, N \uparrow_{(K \cup\{d\})}\right)$ has a covering. It remains to show that any covering $(A, B)$ for $\left(M \uparrow_{(K \cup\{d\})}, N \uparrow_{(K \cup\{d\})}\right)$ is also a packing. Let $(A, B)$ be a covering for $\left(M \uparrow_{(K \cup\{d\})}, N \uparrow_{(K \cup\{d\})}\right)$. Then $(A \backslash\{d\}, B \backslash\{d\})$ is a covering for $\left(M \uparrow_{K}, N \uparrow_{K}\right)$. Since $K$ is a critical set for $(M, N),(A \backslash\{d\}, B \backslash\{d\})$ is also a packing. Since $d \in \mathrm{cl}_{N}(K)$, we have $d \notin B$. So, $d \in A$ and $d \in \mathrm{cl}_{N}(K)$ and this completes the proof that the covering $(A, B)$ is also a packing for $\left(M \uparrow_{(K \cup\{d\})}, N \uparrow_{(K \cup\{d\})}\right)$. So, $K \cup\{d\}$ is a critical set for $(M, N)$ which is a contradiction with $K$ is a maximal critical set for $(M \backslash\{e\}, N \backslash\{e\})$.

If $M_{w}$ is a uniform matroid of co-rank one, then $\hat{C}=E_{w}$. Since $I$ is independent in $M$, we have $\hat{C} \cap J \neq \emptyset$. Therefore $\hat{C} \cap I \cap L$ is independent in $M / K$. Thus $\hat{C} \cap L \subseteq L^{\prime}$. Now, since $\hat{C} \subseteq I^{\prime} \cup\left(I \backslash L^{\prime}\right)$ we get $\hat{C} \cap L \subseteq I^{\prime}$. Since $\hat{C} \backslash L \subseteq K$, we have $\hat{C} \cap L$ is a circuit of $M / K$ which is a contradiction with $I^{\prime}$ is independent in $M / K$. 
Thus, we proved that $I^{\prime} \cup\left(I \backslash L^{\prime}\right)$ is independent in $M$. By a similar argument, it can be shown that $J^{\prime} \cup\left(J \backslash L^{\prime}\right)$ is independent in $N$. Hence,

$$
\left(I^{\prime} \cup\left(I \backslash L^{\prime}\right), J^{\prime} \cup\left(J \backslash L^{\prime}\right)\right)
$$

is a covering for $\left(M \uparrow_{K^{*}}, N \uparrow_{K^{*}}\right)$. Since $K^{*}$ is a critical set for $(M, N)$, the covering

$$
\left(I^{\prime} \cup\left(I \backslash L^{\prime}\right), J^{\prime} \cup\left(J \backslash L^{\prime}\right)\right)
$$

is also a packing. We want to show that $\left(I^{\prime}, J^{\prime}\right)$ is a packing for $\left(M^{\prime} \uparrow_{L^{\prime}}, N^{\prime} \uparrow_{L^{\prime}}\right)$. Let $y \in J^{\prime}$. Then $\{y\}$ is spanned in $M$ by $I^{\prime} \cup\left(I \backslash L^{\prime}\right)$. So, there exists a circuit $C_{y}^{M}$ of $M$ such that

$$
y \in C_{y}^{M} \subseteq I^{\prime} \cup\left(I \backslash L^{\prime}\right) \cup\{y\}
$$

Let $o \in R$ be such that $C_{y}^{M} \subseteq E_{o}$. If $M_{o}$ is a uniform matroid of co-rank one, then $C_{y}^{M}=E_{o}$. We want to show that $C_{y}^{M} \cap L^{\prime}$ is a circuit of $M / K$. Suppose, for a contradiction, that $C_{y}^{M} \cap L^{\prime}$ is not a circuit of $M / K$. This implies that $C_{y}^{M} \backslash\left(L^{\prime} \cup K\right) \neq \emptyset$. Let $q \in C_{y}^{M} \backslash\left(L^{\prime} \cup K\right)$. First observe that $q \notin \mathrm{cl}_{M}(K) \cup \mathrm{cl}_{N}(K)$. So, $q \in L$. But since $q \in I$ and $q \notin L^{\prime}$, there is a circuit $C_{q}$ of $M$ such that $C_{q}$ was removed from $L$. Since $q \in E_{o}$ and $M_{o}$ is a uniform matroid of co-rank one, we have $C_{q}=E_{o}$. Therefore $C_{q}=C_{y}^{M}$. But, $C_{y}^{M} \cap L^{\prime} \neq \emptyset$ and $C_{q} \cap L^{\prime}=\emptyset$ which is a contradiction with $C_{q}=C_{y}^{M}$. Thus $y \in \operatorname{cl}_{M^{\prime}}\left(I^{\prime}\right)$.

If $M_{o}$ is a uniform matroid of rank one, then $C_{y}^{M}=\{y, p\}$ such that $p \in E_{o}$. We want to show that $p \in I^{\prime}$, and hence $C_{y}^{M}$ is a circuit of $M / K$. Since $y \in L^{\prime}$, we have $y \notin \mathrm{cl}_{M}(K)$, so $K \cap E_{o}=\emptyset$. This implies $p \in L$ because otherwise if $p \in \operatorname{cl}_{M}(K) \cup \mathrm{cl}_{N}(K)$ we get a contradiction with $K$ is a maximal critical set for $(M \backslash\{e\}, N \backslash\{e\})$. If $p \notin L^{\prime}$ since $p \in L$, there is a circuit $C_{p}$ of $M$ such that $C_{p}$ was removed from $L$ which implies that $C_{p}$ is a circuit of a uniform matroid of co-rank one, and hence $M_{o}$ is a uniform matroid of co-rank one which is a contradiction with assumption. Thus $y \in \mathrm{cl}_{M^{\prime}}\left(I^{\prime}\right)$. 
So, we proved that $I^{\prime}$ spans $L^{\prime}$ in $M^{\prime}$. Similarly, it can be shown that $J^{\prime}$ spans $L^{\prime}$ in $M^{\prime}$. Hence, the covering $\left(I^{\prime}, J^{\prime}\right)$ is also a packing for $\left(\left.M^{\prime}\right|_{L^{\prime}}, N^{\prime} \uparrow_{L^{\prime}}\right)$. This completes the proof that $L^{\prime}$ is a critical set for $\left(M^{\prime}, N^{\prime}\right)$. Now, by the lemma 5.3.2, we have $K \cup L^{\prime}$ is a critical set for $(M, N)$. Since $e \notin\left(K \cup L^{\prime}\right)$, we get $K \cup L^{\prime}$ is a critical set for $(M \backslash\{e\}, N \backslash\{e\})$ which is a contradiction with $K$ is a maximal critical set for $(M \backslash\{e\}, N \backslash\{e\})$.

Lemma 5.4.2. Let $(M, N)$ be singular, $K^{\prime} \subseteq E \backslash\left\{e_{i}\right\}$ a critical set for $\left(M^{\prime}, N^{\prime}\right)=\left(M \backslash\left\{e_{i}\right\}, N /\left\{e_{i}\right\}\right)$, and $e_{j} \in E \backslash\left(K^{\prime} \cup\left\{e_{i}\right\}\right)$ such that $e_{j} \in \mathrm{cl}_{M^{\prime}}\left(K^{\prime}\right)$ and $e_{j} \in \mathrm{cl}_{N^{\prime}}\left(K^{\prime}\right)$. Then, for any covering $(I, J)$ for $\left(M^{\prime} \uparrow_{K^{\prime}}, N^{\prime} \uparrow_{K^{\prime}}\right), e_{j} \in \mathrm{cl}_{N^{\prime}}(J)$.

Proof. Let $(I, J)$ be a covering for $\left(M^{\prime} \uparrow_{K^{\prime}}, N^{\prime} \uparrow_{K^{\prime}}\right)$. Since $e_{j} \in \mathrm{cl}_{N^{\prime}}\left(K^{\prime}\right)$, there exists a circuit $C^{N^{\prime}}$ of $N^{\prime}$ such that

$$
e_{j} \in C^{N^{\prime}} \subseteq K^{\prime} \cup\left\{e_{j}\right\}
$$

If $C^{N^{\prime}} \cap I=\emptyset$, then $e_{j} \in \mathrm{cl}_{N^{\prime}}(J)$. So, suppose $C^{N^{\prime}} \cap I \neq \emptyset$ and let $x \in C^{N^{\prime}} \cap I$. Since $K^{\prime}$ is a critical set for $\left(M^{\prime}, N^{\prime}\right)$, we have $x \in \mathrm{cl}_{N^{\prime}}(J)$. Let $C$ be the circuit of $N^{\prime}$ such that $x \in C \subseteq J \cup\{x\}$. Since $(M, N)$ is singular, $M=\bigoplus_{r \in R} M_{r}$ with each $M_{r}$ being either a uniform matroid of rank one or a uniform matroid of co-rank one on the set $E_{r}$ and $N=\bigoplus_{s \in S} N_{s}$ with each $N_{s}$ being a either a uniform matroid of rank one or a uniform matroid of co-rank one on the set $E_{s}^{\prime}$. Since $x \in C^{N^{\prime}} \cap C$, there exists some $s \in S$ such that $C^{N^{\prime}} \subseteq E_{s}^{\prime}$ and $C \subseteq E_{s}^{\prime}$. Observe that $N_{s}$ can not be a uniform matroid of co-rank one because otherwise $C^{N^{\prime}}=C=E_{s}^{\prime}$ but $e_{j} \in C^{N^{\prime}}$ and $e_{j} \notin C$ which is a contradiction. So, $N_{s}$ is a uniform matroid of rank one. This implies that $C^{N^{\prime}}$ is also a circuit of $N$. Because otherwise, $C^{N^{\prime}} \cup\left\{e_{i}\right\}$ is a circuit of $N$. Then, we have $\left\{e_{i}, e_{j}, x\right\} \subseteq C^{N^{\prime}}$ which is a contradiction with $N_{s}$ is a uniform matroid of rank 1.

Therefore, $C^{N^{\prime}}=\left\{e_{j}, x\right\}$. If $C \cup\left\{e_{i}\right\}$ is a circuit of the matroid $N$, then $\left\{e_{i}, x\right\}$ is a circuit of $N$. Now, by the circuit elimination axiom and eliminating $x$ from the circuits $C^{N^{\prime}}=\left\{e_{j}, x\right\}$ and $\left\{e_{i}, x\right\}$, we have the set $\left\{e_{i}, e_{j}\right\}$ is a circuit of $N$. Therefore, $\left\{e_{j}\right\}$ is a loop of $N^{\prime}$. But $\left\{e_{j}\right\} \subseteq\left\{e_{j}, x\right\}$ which is a contradiction because both are circuits of $N^{\prime}$. Thus, $C$ is a circuit of the matroid $N$ and $C=\{x, y\}$ for some $y \in J$. Then, by the circuit elimination axiom and 
eliminating $x$ from the circuits $C^{N^{\prime}}$ and $C$, we get $\left\{e_{j}, y\right\}$ is a circuit of $N$. This implies that $e_{j} \in \mathrm{cl}_{N^{\prime}}(J)$.

Definition. Recall Definition 5.1.1. We say $(M, N)$ has a blockage if and only if

- there exists a critical set $K \subseteq E$ for $(M, N)$ and $a \in E \backslash K$ such that $a \in \mathrm{cl}_{M}(K)$ and $a \in \mathrm{cl}_{N}(K)$.

Lemma 5.4.3. Let $(M, N)$ be singular with no blockage. Let $e_{i} \in E$ and $K \subseteq E \backslash\left\{e_{i}\right\}$ be a maximal critical set for $\left(M \backslash\left\{e_{i}\right\}, N \backslash\left\{e_{i}\right\}\right)$. If $e_{i} \notin \mathrm{cl}_{N}(K)$, then

$$
\left(M \backslash\left\{e_{i}\right\}, N /\left\{e_{i}\right\}\right)
$$

also has no blockage and if $e_{i} \notin \mathrm{cl}_{M}(K)$, then

$$
\left(M /\left\{e_{i}\right\}, N \backslash\left\{e_{i}\right\}\right)
$$

also has no blockage.

Proof. Let $e_{i} \in E$ and $K \subseteq E \backslash\left\{e_{i}\right\}$ be a maximal critical set for $\left(M \backslash\left\{e_{i}\right\}, N \backslash\left\{e_{i}\right\}\right)$. We first show that if $e_{i} \notin \mathrm{cl}_{N}(K)$ then,

$$
\left(M^{\prime}, N^{\prime}\right)=\left(M \backslash\left\{e_{i}\right\}, N /\left\{e_{i}\right\}\right)
$$

also has no blockage. Suppose, for a contradiction, that $\left(M^{\prime}, N^{\prime}\right)$ has a blockage. This means there exists a critical set $K^{\prime} \subseteq E \backslash\left\{e_{i}\right\}$ for $\left(M^{\prime}, N^{\prime}\right)$ and $e_{j} \in E \backslash\left(K^{\prime} \cup\left\{e_{i}\right\}\right)$ such that $e_{j} \in \mathrm{cl}_{M^{\prime}}\left(K^{\prime}\right)$ and $e_{j} \in \mathrm{cl}_{N^{\prime}}\left(K^{\prime}\right)$. We want to show that for each of the following cases we get a contradiction.

[A1] $\left(M \uparrow_{\left(K^{\prime} \cup\left\{e_{j}\right\}\right)}, N \uparrow_{\left(K^{\prime} \cup\left\{e_{j}\right\}\right)}\right)$ has a covering.

[A2] $\left(M \uparrow_{\left(K^{\prime} \cup\left\{e_{j}\right\}\right)}, N \uparrow_{\left(K^{\prime} \cup\left\{e_{j}\right\}\right)}\right)$ has no covering.

First, we consider the case [A1]. Let $(I, J)$ be a covering for $\left(M \uparrow_{\left(K^{\prime} \cup\left\{e_{j}\right\}\right)}, N \uparrow_{\left(K^{\prime} \cup\left\{e_{j}\right\}\right)}\right)$. We want to show that $e_{i} \in \mathrm{cl}_{N}(J)$. 
We have $\left(I \backslash\left\{e_{j}\right\}, J \backslash\left\{e_{j}\right\}\right)$ is a covering for $\left(M \uparrow_{K^{\prime}}, N \uparrow_{K^{\prime}}\right)$. If

$$
J \cup\left\{e_{i}\right\} \backslash\left\{e_{j}\right\}
$$

is dependent in $N$, then $e_{i} \in \operatorname{cl}_{N}\left(J \backslash\left\{e_{j}\right\}\right)$ and hence $e_{i} \in \mathrm{cl}_{N}(J)$. Otherwise,

$$
J \cup\left\{e_{i}\right\} \backslash\left\{e_{j}\right\}
$$

is independent in $N$. Then, $\left(I \backslash\left\{e_{j}\right\}, J \backslash\left\{e_{j}\right\}\right)$ is a covering for $\left(M^{\prime} \uparrow_{K^{\prime}}, N^{\prime} \uparrow_{K^{\prime}}\right)$. Now, by the lemma 5.4.2 we have $e_{j} \in \mathrm{cl}_{N^{\prime}}\left(J \backslash\left\{e_{j}\right\}\right)$. Therefore, there exists a circuit $C$ of $N^{\prime}$ such that

$$
e_{j} \subseteq C \subseteq J \cup\left\{e_{j}\right\}
$$

If $e_{j} \in J$, then since $J$ is independent in $N$ we have $C \cup\left\{e_{i}\right\}$ is a circuit of $N$. This implies that $e_{i} \in \mathrm{cl}_{N}(J)$. Now, we show that $e_{j} \notin I$.

Suppose, for a contradiction, that $e_{j} \in I$. Since $e_{j} \in \mathrm{cl}_{M^{\prime}}\left(K^{\prime}\right)$, there exists a circuit $C^{M}$ of $M$ such that $e_{j} \in C^{M} \subseteq K^{\prime} \cup\left\{e_{j}\right\}$. Observe that $C^{M} \cap J \neq \emptyset$ and let $x^{\prime} \in C^{M} \cap J$. Since $\left(I \backslash\left\{e_{j}\right\}, J \backslash\left\{e_{j}\right\}\right)$ is a covering for $\left(M^{\prime} \uparrow_{K^{\prime}}, N^{\prime} \uparrow_{K^{\prime}}\right)$ and $K^{\prime}$ is a critical set for $\left(M^{\prime}, N^{\prime}\right)$ we have $\left(I \backslash\left\{e_{j}\right\}, J \backslash\left\{e_{j}\right\}\right)$ is a packing. Hence, $x^{\prime} \in \operatorname{cl}_{M}\left(I \backslash\left\{e_{j}\right\}\right)$. So, there exists a circuit $C_{x^{\prime}}$ of $M$ such that

$$
x^{\prime} \in C_{x^{\prime}} \subseteq\left(I \backslash\left\{e_{j}\right\}\right) \cup\{x\} .
$$

Since $(M, N)$ is singular, $M=\bigoplus_{r \in R} M_{r}$ with each $M_{r}$ being either a uniform matroid of rank one or a uniform matroid of co-rank one on the set $E_{r}$ and $N=\bigoplus_{s \in S} N_{s}$ with each $N_{s}$ being either a uniform matroid of rank one or a uniform matroid of co-rank one on the set $E_{s}^{\prime}$. Since $x^{\prime} \in C_{x^{\prime}} \cap C^{M}$, there exists some $r \in R$ such that $C^{M} \subseteq E_{r}$ and $C_{x^{\prime}} \subseteq E_{r}$. If $M_{r}$ is a uniform matroid of rank one, then $C^{M}=\left\{e_{j}, x^{\prime}\right\}$. By the circuit elimination axiom and eliminating $x^{\prime}$ from the circuits $C^{M}$ and $C_{x^{\prime}}$ we get a circuit of $M$ in $I$ which is a contradiction with $I$ is independent in $M$. If $M_{r}$ is a uniform matroid of co-rank one, then $C^{M}=E_{r}=C_{x^{\prime}}$. But, 
$e_{j} \in C^{M}$ and $e_{j} \notin C_{x^{\prime}}$ which is a contradiction with $C^{M}=C_{x^{\prime}}$. Therefore, we proved that $e_{j} \notin I$ and hence $e_{i} \in \mathrm{cl}_{N}(J)$ for any covering $(I, J)$ for $\left(M \uparrow_{\left(K^{\prime} \cup\left\{e_{j}\right\}\right)}, N \uparrow_{\left(K^{\prime} \cup\left\{e_{j}\right\}\right)}\right)$.

Now, let $\left(I^{\prime}, J^{\prime}\right)$ be a covering for $\left(M \uparrow_{\left(K^{\prime} \cup\left\{e_{j}\right\}\right)}, N \uparrow_{\left(K^{\prime} \cup\left\{e_{j}\right\}\right)}\right)$ and $C^{N}$ be the circuit of $N$ such that

$$
e_{i} \in C^{N} \subseteq J^{\prime} \cup\left\{e_{i}\right\}
$$

We want to show that for any covering $\left(I^{\prime \prime}, J^{\prime \prime}\right)$ for $\left(M \uparrow_{\left(K^{\prime} \cup\left\{e_{j}\right\}\right)}, N \uparrow_{\left(K^{\prime} \cup\left\{e_{j}\right\}\right)}\right)$ we have

$$
C^{N} \backslash\left\{e_{i}\right\} \subseteq \mathrm{cl}_{N}\left(J^{\prime \prime}\right)
$$

Let $\left(I^{\prime \prime}, J^{\prime \prime}\right)$ be a covering for $\left(M \uparrow_{\left(K^{\prime} \cup\left\{e_{j}\right\}\right)}, N \uparrow_{\left(K^{\prime} \cup\left\{e_{j}\right\}\right)}\right)$. Suppose, for a contradiction, that there exists some

$$
b \in C^{N} \backslash\left(\operatorname{cl}_{N}\left(J^{\prime \prime}\right) \cup\left\{e_{i}\right\}\right)
$$

So, $b \in I^{\prime \prime}$ and $J^{\prime \prime} \cup\{b\}$ is independent in $N$. Then,

$$
\left(I^{\prime \prime} \backslash\left\{e_{j}\right\}, J^{\prime \prime} \cup\{b\} \backslash\left\{e_{j}\right\}\right)
$$

is a covering for $\left(M \uparrow_{K^{\prime}}, N \uparrow_{K^{\prime}}\right)$ but it is not a covering for $\left(M^{\prime} \uparrow_{K^{\prime}}, N^{\prime} \uparrow_{K^{\prime}}\right)$ because $K^{\prime}$ is a critical set for $\left(M^{\prime}, N^{\prime}\right)$ and

$$
\left(I^{\prime \prime} \backslash\left\{e_{j}\right\}, J^{\prime \prime} \cup\{b\} \backslash\left\{e_{j}\right\}\right)
$$

is not a packing. This implies that $J^{\prime \prime} \cup\{b\} \backslash\left\{e_{j}\right\}$ is dependent in $N^{\prime}$. So, there exists a circuit $C_{b}$ of $N^{\prime}$ such that

$$
b \in C_{b} \subseteq J^{\prime \prime} \cup\{b\} \backslash\left\{e_{j}\right\}
$$

Since $J^{\prime \prime} \cup\{b\} \backslash\left\{e_{j}\right\}$ is independent in $N$, the set $C_{b} \cup\left\{e_{i}\right\}$ is a circuit of $N$. We also know that $e_{i} \in \mathrm{cl}_{N}\left(J^{\prime \prime}\right)$. So, there exists a circuit $\bar{C}$ of $N$ such that

$$
e_{i} \in \bar{C} \subseteq J^{\prime \prime} \cup\left\{e_{i}\right\}
$$


Now, by the circuit elimination axiom and eliminating $e_{i}$ from the circuits $\bar{C}$ and $C_{b} \cup\left\{e_{i}\right\}$, we get a circuit $\overline{C_{0}}$ of $N$ such that $\overline{C_{0}} \subseteq\left(\bar{C} \cup C_{b}\right) \backslash\left\{e_{i}\right\}$. This implies that $\overline{C_{0}} \subseteq J^{\prime \prime} \cup\{b\}$ which is a contradiction with $J^{\prime \prime} \cup\{b\}$ is independent in $N$.

Thus, we proved that for any covering $\left(I^{\prime \prime}, J^{\prime \prime}\right)$ for $\left(M \uparrow_{\left(K^{\prime} \cup\left\{e_{j}\right\}\right)}, N \uparrow_{\left(K^{\prime} \cup\left\{e_{j}\right\}\right)}\right)$, we have

$$
C^{N} \backslash\left\{e_{i}\right\} \subseteq \mathrm{cl}_{N}\left(J^{\prime \prime}\right)
$$

Now, by the lemma 5.3.3, there exists a critical set $K^{*} \subseteq K^{\prime} \cup\left\{e_{j}\right\}$ for $\left(M \uparrow_{\left(K^{\prime} \cup\left\{e_{j}\right\}\right)}, N \uparrow_{\left(K^{\prime} \cup\left\{e_{j}\right\}\right)}\right)$ such that

$$
C^{N} \backslash\left\{e_{i}\right\} \subseteq K^{*}
$$

This implies that $e_{i} \in \mathrm{cl}_{N}\left(K^{*}\right)$ where $K^{*}$ is also a critical set for $(M, N)$. Since $e_{i} \notin K^{*}$, we have $K^{*}$ is a critical set for $\left(M \backslash\left\{e_{i}\right\}, N \backslash\left\{e_{i}\right\}\right)$ and $e_{i} \in \mathrm{cl}_{N}\left(K^{*}\right)$. By the lemma 5.3.3, this is a contradiction with $K \subseteq E \backslash\left\{e_{i}\right\}$ being a maximal critical set for $\left(M \backslash\left\{e_{i}\right\}, N \backslash\left\{e_{i}\right\}\right)$ with $e_{i} \notin \mathrm{cl}_{N}(K)$.

Now, we consider the case [A2] that $\left(M \uparrow_{\left(K^{\prime} \cup\left\{e_{j}\right\}\right)}, N \uparrow_{\left(K^{\prime} \cup\left\{e_{j}\right\}\right)}\right)$ has no covering. Let $(\hat{I}, \hat{J})$ be a covering for $\left(M^{\prime} \uparrow_{K^{\prime}}, N^{\prime} \uparrow_{K^{\prime}}\right)$. Observe that $(\hat{I}, \hat{J})$ is also a covering for $\left(M \uparrow_{K^{\prime}}, N \uparrow_{K^{\prime}}\right)$. Since $\left(M \uparrow_{\left(K^{\prime} \cup\left\{e_{j}\right\}\right)}, N \uparrow_{\left(K^{\prime} \cup\left\{e_{j}\right\}\right)}\right)$ has no covering, $\hat{J} \cup\left\{e_{j}\right\}$ is dependent in $N$. So, there exists a circuit $\hat{C}$ of $N$ such that

$$
e_{j} \in \hat{C} \subseteq \hat{J} \cup\left\{e_{j}\right\}
$$

Since by the assumption, $e_{j} \in \mathrm{cl}_{N^{\prime}}\left(K^{\prime}\right)$, there exists a circuit $C^{N^{\prime}}$ of $N^{\prime}$ such that

$$
e_{j} \in C^{N^{\prime}} \subseteq K^{\prime} \cup\left\{e_{j}\right\}
$$

Here, we want to show that $C^{N^{\prime}}$ is also a circuit of $N$. Suppose, for a contradiction, that $C^{N^{\prime}} \cup\left\{e_{i}\right\}$ is a circuit of $N$. Since $e_{j} \in \hat{C} \cap C^{N^{\prime}}$, there is some $s_{j} \in S$ such that $\hat{C} \subseteq E_{s_{j}}^{\prime}$ and $C^{N^{\prime}} \subseteq E_{s_{j}}^{\prime}$. If $N_{s_{j}}$ is a uniform matroid of rank one, then $C^{N^{\prime}} \cup\left\{e_{i}\right\}=\left\{e_{j}, e_{i}\right\}$ and $\hat{C}=\left\{e_{j}, c\right\}$ for some $c \in \hat{J}$. Now, by the circuit elimination axiom and eliminating $e_{j}$ from the circuits $\hat{C}$ and $C^{N^{\prime}}$, we have 
the set $\left\{e_{i}, c\right\}$ is a circuit of $N$. Hence, $\{c\}$ is a loop of $N^{\prime}$. This is a contradiction because $\{c\} \subseteq \hat{J}$ and $\hat{J}$ is independent in $N^{\prime}$. If $N_{s_{j}}$ is a uniform matroid of co-rank one, then $C^{N^{\prime}} \cup\left\{e_{i}\right\}=E_{s_{j}}^{\prime}=\hat{C}$. But $e_{i} \in \hat{C}$ which is a contradiction with $C^{N^{\prime}} \cup\left\{e_{i}\right\}=\hat{C}$. Thus, we proved that $C^{N^{\prime}}$ is a circuit of $N$.

Now, we want to show that for any covering $\left(I^{\prime \prime \prime}, J^{\prime \prime \prime}\right)$ for $\left(M \uparrow_{K^{\prime}}, N \uparrow_{K^{\prime}}\right)$, we have

$$
C^{N^{\prime}} \backslash\left\{e_{j}\right\} \subseteq \mathrm{cl}_{N}\left(J^{\prime \prime \prime}\right)
$$

Suppose, for a contradiction, that there exists a covering $\left(I^{\prime \prime \prime}, J^{\prime \prime \prime}\right)$ for $\left(M \uparrow_{K^{\prime}}, N \uparrow_{K^{\prime}}\right)$ and

$$
d \in C^{N^{\prime}} \backslash\left(\mathrm{cl}_{N}\left(J^{\prime \prime \prime}\right) \cup\left\{e_{j}\right\}\right)
$$

So, $d \in I^{\prime \prime \prime}$ and $d \notin \mathrm{cl}_{N}\left(J^{\prime \prime \prime}\right)$. Observe that $\left(M \uparrow_{\left(K^{\prime} \cup\left\{e_{j}\right\}\right)}, N \uparrow_{\left(K^{\prime} \cup\left\{e_{j}\right\}\right)}\right)$ has no covering implies that $J^{\prime \prime \prime} \cup\left\{e_{j}\right\}$ is dependent in $N$. So, there exists a circuit $\widetilde{C}$ of $N$ such that

$$
e_{j} \in \widetilde{C} \subseteq J^{\prime \prime \prime} \cup\left\{e_{j}\right\}
$$

Now, since $e_{j} \in C^{N^{\prime}} \cap \widetilde{C}$, there exists some $s_{0} \in S$ such that $C^{N^{\prime}} \subseteq E_{s_{0}}^{\prime}$ and $\widetilde{C} \subseteq E_{s_{0}}^{\prime}$. If $N_{s_{0}}$ is a uniform matroid of rank one, then $C^{N^{\prime}}=\left\{e_{j}, d\right\}$ and $\widetilde{C}=\left\{e_{j}, f\right\}$ for some $f \in J^{\prime \prime \prime}$. Now, by the circuit elimination axiom and eliminating $e_{j}$ from the circuits $C^{N^{\prime}}$ and $\widetilde{C}$, we have $\{d, f\}$ is a circuit of $N$. This implies that $d \in \mathrm{cl}_{N}\left(J^{\prime \prime \prime}\right)$ which is a contradiction with the assumption. If $N_{s_{0}}$ is a uniform matroid of co-rank one, then $C^{N^{\prime}}=E_{s_{0}}^{\prime}=\widetilde{C}$. But $d \in C^{N^{\prime}}$ and $d \notin \widetilde{C}$ which is a contradiction with $C^{N^{\prime}}=\widetilde{C}$. Thus, we proved that for any covering $\left(I^{\prime \prime \prime}, J^{\prime \prime \prime}\right)$ for $\left(M \uparrow_{K^{\prime}}, N \uparrow_{K^{\prime}}\right)$, we have

$$
C^{N^{\prime}} \backslash\left\{e_{j}\right\} \subseteq \mathrm{cl}_{N}\left(J^{\prime \prime \prime}\right)
$$

Now, by the lemma 5.3.3, there exists a critical set $K_{1} \subseteq K^{\prime}$ for $\left(M \uparrow_{K^{\prime}}, N \uparrow_{K^{\prime}}\right)$ such that

$$
C^{N^{\prime}} \backslash\left\{e_{j}\right\} \subseteq K_{1}
$$


This implies that $e_{j} \in \mathrm{cl}_{N}\left(K_{1}\right)$. Now, we want to show that there exists a critical set $K_{2} \subseteq K^{\prime}$ for $(M, N)$ such that $e_{j} \in \mathrm{cl}_{M}\left(K_{2}\right)$.

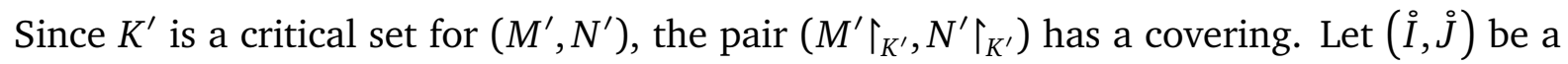
covering for $\left(M^{\prime} \uparrow_{K^{\prime}}, N^{\prime} \uparrow_{K^{\prime}}\right)$. Since $J$ is independent in $N^{\prime}$, we have $J \cup\left\{e_{i}\right\}$ is independent in

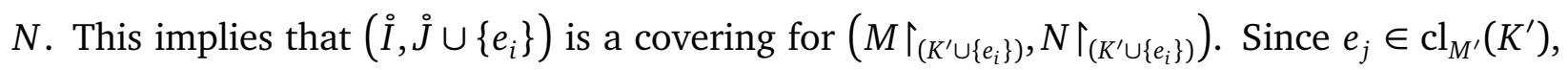
there exists a circuit $C^{M}$ of $M$ such that $e_{j} \in C^{M} \subseteq K^{\prime} \cup\left\{e_{j}\right\}$. Now, we want to show that for any covering $\left(I^{*}, J^{*}\right)$ for $\left(M \uparrow_{\left(K^{\prime} \cup\left\{e_{i}\right\}\right)}, N \uparrow_{\left(K^{\prime} \cup\left\{e_{i}\right\}\right)}\right)$, we have $C^{M} \backslash\left\{e_{j}\right\} \subseteq \mathrm{cl}_{M}\left(I^{*}\right)$.

Let $\left(I^{*}, J^{*}\right)$ be a covering for $\left(M \uparrow_{\left(K^{\prime} \cup\left\{e_{i}\right\}\right)}, N \uparrow_{\left(K^{\prime} \cup\left\{e_{i}\right\}\right)}\right)$. Since $J^{*}$ is independent in $N \uparrow_{\left(K^{\prime} \cup\left\{e_{i}\right\}\right.}$, we have $J^{*}$ is independent in $N^{\prime}$. Therefore, $\left(I^{*} \backslash\left\{e_{i}\right\}, J^{*} \backslash\left\{e_{i}\right\}\right)$ is a covering for $\left(M^{\prime} \uparrow_{K^{\prime}}, N^{\prime} \uparrow_{K^{\prime}}\right)$. Since $K^{\prime}$ is a critical set for $\left(M^{\prime}, N^{\prime}\right)$, we have $\left(I^{*} \backslash\left\{e_{i}\right\}, J^{*} \backslash\left\{e_{i}\right\}\right)$ is also a packing. Let $g \in C^{M} \backslash\left(I^{*} \cup\left\{e_{j}\right\}\right)$. Since $I^{*} \backslash\left\{e_{i}\right\}$ spans $K^{\prime}$ in $M, g \in \operatorname{cl}_{M}\left(I^{*} \backslash\left\{e_{i}\right\}\right)$ which implies that $g \in \mathrm{cl}_{M}\left(I^{*}\right)$. This completes the proof that

$$
C^{M} \backslash\left\{e_{j}\right\} \subseteq \mathrm{cl}_{M}\left(I^{*}\right)
$$

for any covering $\left(I^{*}, J^{*}\right)$ for $\left(M \uparrow_{\left(K^{\prime} \cup\left\{e_{i}\right\}\right)}, N \uparrow_{\left(K^{\prime} \cup\left\{e_{i}\right\}\right)}\right)$. Now, by the lemma 5.3.3, there exists a critical set $K_{2} \subseteq K^{\prime} \cup\left\{e_{i}\right\}$ for $\left(M \uparrow_{\left(K^{\prime} \cup\left\{e_{i}\right\}\right)}, N \uparrow_{\left(K^{\prime} \cup\left\{e_{i}\right\}\right)}\right)$ such that

$$
C^{M} \backslash\left\{e_{j}\right\} \subseteq K_{2}
$$

This implies that $e_{j} \in \mathrm{cl}_{M}\left(K_{2}\right)$. Since $\left(M \uparrow_{\left(K^{\prime} \cup\left\{e_{i}\right\}\right)}, N \uparrow_{\left(K^{\prime} \cup\left\{e_{i}\right\}\right)}\right)$ has a covering, by the lemma 4.4.10, $\bar{K}=K_{1} \cup K_{2}$ is a critical set for $\left(M \uparrow_{\left(K^{\prime} \cup\left\{e_{i}\right\}\right)}, N \uparrow_{\left(K^{\prime} \cup\left\{e_{i}\right\}\right)}\right)$. So, we have $\bar{K}$ is a critical set for $(M, N)$ with

$$
e_{j} \in \mathrm{cl}_{M}(\bar{K}) \text { and } e_{j} \in \mathrm{cl}_{N}(\bar{K}) \text {. }
$$

This is a contradiction with $(M, N)$ has no blockage. This completes the proof that if $e_{i} \notin \mathrm{cl}_{N}(K)$, then

$$
\left(M \backslash\left\{e_{i}\right\}, N /\left\{e_{i}\right\}\right)
$$


also has no blockage. By a similar argument, it can be shown that if $e_{i} \notin \mathrm{cl}_{M}(K)$, then

$$
\left(M /\left\{e_{i}\right\}, N \backslash\left\{e_{i}\right\}\right)
$$

also has no blockage.

Lemma 5.4.4. Let $(M, N)$ be singular, $e \in E$, and $K \subseteq E \backslash\{e\}$ a maximal critical set for $(M \backslash\{e\}, N \backslash\{e\})$ such that $e \notin \mathrm{cl}_{N}(K)$ and $\left(M^{\prime}, N^{\prime}\right)=(M \backslash\{e\}, N /\{e\})$. Then if $e \in E_{s}^{\prime}$ such that $N_{s}$ is a uniform matroid of co-rank one and $E_{s}^{\prime}$ is infinite, there exists some $v \in E_{s}^{\prime} \backslash\{e\}$ such that $K$ is also a maximal critical set for $\left(M^{\prime} \backslash\{v\}, N^{\prime} \backslash\{v\}\right)$ and $v \notin \mathrm{cl}_{M^{\prime}}(K)$.

Proof. Let $s \in S$ be such that $e \in E_{s}^{\prime}$ and assume that $N_{s}$ is a uniform matroid of co-rank one and $E_{s}^{\prime}$ is an infinite set. Since $e \notin \mathrm{cl}_{N}(K)$, there exists an element $v \in E_{s}^{\prime}$ such that $v \notin K$. We first show that

$$
v \notin \mathrm{cl}_{M}(K) \text { and } v \notin \mathrm{cl}_{N}(K)
$$

Since $e \notin K$, so $v \notin \mathrm{cl}_{N}(K)$. So, $K \cup\{v\}$ is independent in $N$. Now suppose, for a contradiction, that $v \in \mathrm{cl}_{M}(K)$. Let $(I, J)$ be a covering for $\left(M \uparrow_{K}, N \uparrow_{K}\right)$. Since $K$ is a critical set for $(M, N)$, we have $(I, J)$ is also a packing for $\left(M \uparrow_{K}, N \uparrow_{K}\right)$. So $v \in \mathrm{cl}_{M}(K)$ implies that $v \in \mathrm{cl}_{M}(I)$. Since $v \notin \mathrm{cl}_{N}(K)$, for any covering $(I, J)$ for $\left(M \uparrow_{K}, N \uparrow_{K}\right)$, the pair $(I, J \cup\{v\})$ is a covering for $\left(M \uparrow_{(K \cup\{v\})}, N \uparrow_{(K \cup\{v\})}\right)$. We want to show that $K \cup\{v\}$ is a critical set for $(M, N)$. Since $\left(M \uparrow_{(K \cup\{v\})}, N \uparrow_{(K \cup\{v\})}\right)$ has a covering, it remains to show that each of its covering is also a packing. Let $(\bar{I}, \bar{J})$ be a covering for $\left(M \uparrow_{(K \cup\{v\})}, N \uparrow_{(K \cup\{v\})}\right)$. Observe that $(\bar{I} \backslash\{v\}, \bar{J} \backslash\{v\})$ is a covering for $\left(M \uparrow_{K}, N \uparrow_{K}\right)$. Since $K$ is a critical set for $(M, N)$ and $v \in \operatorname{cl}_{M}(K)$ we have

$$
v \in \mathrm{cl}_{M}(\bar{I} \backslash\{v\})
$$

This implies that $v \in \bar{J}$. On the other hand, since $K$ is a critical set for $(M, N)$, we have $(\bar{I} \backslash\{v\}, \bar{J} \backslash\{v\})$ is also a packing. Therefore, we have 


$$
\begin{aligned}
& v \in \operatorname{cl}_{M}(\bar{I}) \\
& K \in \operatorname{cl}_{M}(\bar{I} \backslash\{v\}) \\
& K \in \operatorname{cl}_{N}(\bar{J} \backslash\{v\}) .
\end{aligned}
$$

This implies that $(\bar{I}, \bar{J})$ is a packing for $\left(M \uparrow_{(K \cup\{v\})}, N \uparrow_{(K \cup\{v\})}\right)$. This completes the proof that $K \cup\{v\}$ is a critical set for $(M, N)$. Now, since $e \notin K \cup\{v\}$, we have $K \cup\{v\}$ is a critical set for for $(M \backslash\{e\}, N \backslash\{e\})$ which is a contradiction with $K$ is a maximal critical set for $(M \backslash\{e\}, N \backslash\{e\})$. So, we proved that $v \notin \mathrm{cl}_{M}(K)$ and $v \notin \mathrm{cl}_{N}(K)$.

Let $\left(M^{\prime}, N^{\prime}\right)=(M \backslash\{e\}, N /\{e\})$. We want to show that $K$ is also a maximal critical set for $\left(M^{\prime} \backslash\{v\}, N^{\prime} \backslash\{v\}\right)$. We first show that $K$ is a critical set for $\left(M^{\prime}, N^{\prime}\right)$. Let $(I, J)$ be a covering for $\left(M \uparrow_{K}, N \uparrow_{K}\right)$. Since $I$ is independent in $M$, it is independent in $M^{\prime}=M \backslash\{e\}$. Since $v \notin J$, the set $J$ is independent in $N^{\prime}=N /\{e\}$. Thus, $(I, J)$ is a covering for $\left(M^{\prime} \uparrow_{K}, N^{\prime} \uparrow_{K}\right)$. It remains to show that every covering is a packing. Let $\left(I^{\prime}, J^{\prime}\right)$ be a covering for $\left(M^{\prime} \uparrow_{K}, N^{\prime} \uparrow_{K}\right)$. Then $\left(I^{\prime}, J^{\prime}\right)$ is a covering for $\left(M \uparrow_{K}, N \uparrow_{K}\right)$. Since $K$ is a critical set for $(M, N)$, the pair $\left(I^{\prime}, J^{\prime}\right)$ is also a packing for $\left(M \uparrow_{K}, N \uparrow_{K}\right)$. Since $I^{\prime}$ spans $K$ in $M$, it also spans $K$ in $M^{\prime}$. Since $J^{\prime}$ spans $K$ in $N$ and $v \notin K$, $J^{\prime}$ spans $K$ in $N^{\prime}=N /\{e\}$. This implies that $\left(I^{\prime}, J^{\prime}\right)$ is also a packing for $\left(M^{\prime} \uparrow_{K}, N^{\prime} \uparrow_{K}\right)$. Thus, $K$ is also a critical set for $\left(M^{\prime}, N^{\prime}\right)$. Observe that $K$ is a maximal critical set for $\left(M^{\prime} \backslash\{v\}, N^{\prime} \backslash\{v\}\right)$ because otherwise if it can be extended to a larger critical set $\bar{K}$ with $K \subseteq \bar{K}$, then $\bar{K}$ is also a critical set for $(M \backslash\{e\}, N \backslash\{e\})$ which is a contradiction with $K$ is a maximal critical set for $(M \backslash\{e\}, N \backslash\{e\})$. Now, since $v \notin \mathrm{cl}_{M}(K)$, we have $v \notin \mathrm{cl}_{M^{\prime}}(K)$.

By a similar argument as in the proof of the lemma 5.4.4, we have the following result.

Lemma 5.4.5. Let $(M, N)$ be singular, $e \in E$, and $K \subseteq E \backslash\{e\}$ a maximal critical set for $(M \backslash\{e\}, N \backslash\{e\})$ such that $e \notin \mathrm{cl}_{M}(K)$ and $\left(M^{\prime}, N^{\prime}\right)=(M /\{e\}, N \backslash\{e\})$. Then if $e \in E_{r}$ such that $M_{r}$ is a uniform matroid of co-rank 1 and $E_{r}$ is infinite, there exists some $w \in E_{r} \backslash\{e\}$ such that $K$ is also a maximal critical set for $\left(M^{\prime} \backslash\{w\}, N^{\prime} \backslash\{w\}\right)$ and $w \notin \mathrm{cl}_{N^{\prime}}(K)$.

Now we are ready to prove Theorem 5.1.4. 
Proof of Theorem 5.1.4. First we show that for any arbitrary matroids $(M, N)$ on any common set $E$, (1) always implies (2). Assume that $(M, N)$ has a covering and a blockage. So, there exists a critical set $K \subseteq E$ for $(M, N)$ and $a \in E \backslash K$ such that $a \in \operatorname{cl}_{M}(K)$ and $a \in \operatorname{cl}_{N}(K)$. Let $(I, J)$ be a covering for $(M, N)$. Then, $(I \cap K, J \cap K)$ is a covering for $\left(M \uparrow_{K}, N \uparrow_{K}\right)$. Since $K$ is a critical set for $(M, N)$, the covering $(I \cap K, J \cap K)$ is also a packing. Since $a \in \mathrm{cl}_{M}(K)$ and $a \in \mathrm{cl}_{N}(K)$ and $(I \cap K, J \cap K)$ is a packing, we have $a \in \operatorname{cl}_{M}(I \cap K)$ and $a \in \operatorname{cl}_{N}(J \cap K)$. Now, if $a \in I$, since $a \in \mathrm{cl}_{M}(I \cap K)$ we get a contradiction with $I$ is independent in $M$. If $a \in J$, since $a \in \mathrm{cl}_{N}(J \cap K)$ we get a contradiction with $J$ is independent in $N$.

Now we want to show that (2) implies (1). Assume that $(M, N)$ is singular on an infinite countable set $E$ and has no blockage. Let $E=\left\{e_{i}: i \in \mathbb{N}\right\}$. Using induction, we want to construct a covering $(A, B)$ for $(M, N)$. Since $(M, N)$ is singular, $M=\bigoplus_{r \in R} M_{r}$ with each $M_{r}$ being either a uniform matroid of rank one or a uniform matroid of co-rank one on the set $E_{r}$ and $N=\bigoplus_{s \in S} N_{s}$ with each $N_{s}$ being a either a uniform matroid of rank one or a uniform matroid of co-rank one on the set $E_{s}^{\prime}$. Let

$$
R^{\prime}=\left\{r \in R: M_{r} \text { is an infinite co-rank one matroid }\right\}
$$

and

$$
S^{\prime}=\left\{s \in S: N_{s} \text { is an infinite co-rank one matroid }\right\}
$$

Since $R$ and $S$ are countable sets, we can enumerate the set $R^{\prime} \cup S^{\prime}=\{t: t \in T\}$ such that either $T=\mathbb{N}$ or $T=\{1, \ldots, n\}$ for some $n \in \mathbb{N}$. By induction on $i \in \mathbb{N}$, we will define a pair $\left(A_{i}, B_{i}\right)$ of finite disjoint subsets of $E$ such that

$$
\left\{e_{1}, \cdots, e_{i-1}\right\} \subseteq A_{i} \cup B_{i}
$$

and a pair $\left(M_{i}, N_{i}\right)$ of matroids on the common set $E_{i}=E \backslash\left(A_{i} \cup B_{i}\right)$ such that $\left(M_{i}, N_{i}\right)$ has no blockage. Then we take $A=\bigcup_{i \in \mathbb{N}} A_{i}$ and $B=\bigcup_{i \in \mathbb{N}} B_{i}$ and we will show that $(A, B)$ is a covering for 
$(M, N)$.

Let $A_{1}=B_{1}=\emptyset$ and $\left(M_{1}, N_{1}\right)=(M, N)$. Now, suppose the pair $\left(A_{i}, B_{i}\right)$ and the matroids $\left(M_{i}, N_{i}\right)$ on the set $E_{i}$ without a blockage are defined. If $e_{i} \in A_{i} \cup B_{i}$, then let

$$
\begin{aligned}
\left(A_{i+1}, B_{i+1}\right) & =\left(A_{i}, B_{i}\right), \\
\left(M_{i+1}, N_{i+1}\right) & =\left(M_{i}, N_{i}\right), \\
E_{i+1} & =E_{i} .
\end{aligned}
$$

Otherwise, let the set $K_{i} \subseteq E_{i} \backslash\left\{e_{i}\right\}$ be a maximal critical set for $\left(M_{i} \backslash\left\{e_{i}\right\}, N_{i} \backslash\left\{e_{i}\right\}\right)$. Since $\left(M_{i}, N_{i}\right)$ has no blockage, we can not have both $e_{i} \in \mathrm{cl}_{M_{i}}\left(K_{i}\right)$ and $e_{i} \in \mathrm{cl}_{N_{i}}\left(K_{i}\right)$. We are going to consider the following cases:

Case1 $e_{i} \notin \operatorname{cl}_{N_{i}}\left(K_{i}\right)$

Case2 $e_{i} \in \mathrm{cl}_{N_{i}}\left(K_{i}\right)$

First, assume that we have the [Case1] that $e_{i} \notin \mathrm{cl}_{N_{i}}\left(K_{i}\right)$. We perform an induction that stops after finitely many steps and as a result of this induction, we obtain a finite subset $V_{i}=\left\{v_{i}^{0}, v_{i}^{1}, v_{i}^{2}, \ldots, v_{i}^{n-1}\right\}$ of $E$ such that $v_{0}=e_{i}$ and

$$
A_{i+1} \cup B_{i+1}=A_{i} \cup B_{i} \cup V_{i}
$$

and a pair of matroids $\left(M_{i}^{j}, N_{i}^{j}\right)$ on the set $E_{i}^{j}=E_{i} \backslash\left\{v_{i}^{0}, v_{i}^{1}, v_{i}^{2}, \ldots, v_{i}^{j-1}\right\}$ for each $1 \leq j \leq n$ such that it has no blockage and a pair $\left(A_{i}^{j}, B_{i}^{j}\right)$ of finite disjoint subsets of $E$.

Let $v_{i}^{0}=e_{i}$ and

$$
\begin{aligned}
\left(A_{i}^{1}, B_{i}^{1}\right) & =\left(A_{i}, B_{i} \cup\left\{v_{i}^{0}\right\}\right) \\
\left(M_{i}^{1}, N_{i}^{1}\right) & =\left(M_{i} \backslash\left\{v_{i}^{0}\right\}, N_{i} /\left\{v_{i}^{0}\right\}\right) .
\end{aligned}
$$

Then by the lemma 5.4.3, $\left(M_{i}^{1}, N_{i}^{1}\right)$ also has no blockage. If $v_{i}^{0} \notin E_{t}^{\prime}$ for any $t \in T$, then let

$$
n=1 \text {. }
$$


Otherwise, $v_{i}^{0} \in E_{t_{1}}^{\prime}$ for some $t_{1} \in T$, then by the lemma 5.4.4, there exists some $v_{i}^{1} \in E_{t_{1}}^{\prime} \backslash\left\{v_{i}^{0}\right\}$ such that $K_{i}$ is also a maximal critical set for $\left(M_{i}^{1} \backslash\left\{v_{i}^{1}\right\}, N_{i}^{1} \backslash\left\{v_{i}^{1}\right\}\right)$ and $v_{i}^{1} \notin \mathrm{cl}_{M_{i}^{1}}\left(K_{i}\right)$. Then let

$$
\begin{aligned}
\left(A_{i}^{2}, B_{i}^{2}\right) & =\left(A_{i} \cup\left\{v_{i}^{1}\right\}, B_{i} \cup\left\{v_{i}^{0}\right\}\right), \\
\left(M_{i}^{2}, N_{i}^{2}\right) & =\left(M_{i}^{1} /\left\{v_{i}^{1}\right\}, N_{i}^{1} \backslash\left\{v_{i}^{1}\right\}\right) .
\end{aligned}
$$

Since $\left(M_{i}^{1}, N_{i}^{1}\right)$ has no blockage, by the lemma 5.4.3, $\left(M_{i}^{2}, N_{i}^{2}\right)$ also has no blockage. If $v_{i}^{1} \notin E_{t}$ for any $t \in T$, or if $v_{i}^{1} \in E_{t}$ for some $t \in T$ such that $t>t_{1}$, then let

$$
n=2 .
$$

Now, suppose that the matroids $\left(M_{i}^{j}, N_{i}^{j}\right)$ on the set $E_{i}^{j}=E_{i} \backslash\left\{v_{i}^{0}, v_{i}^{1}, v_{i}^{2}, \ldots, v_{i}^{j-1}\right\}$ without a blockage and the finite sets $\left(A_{i}^{j}, B_{i}^{j}\right)$ are defined. We want to either define the element $v_{j}$, the matroids $\left(M_{i}^{j+1}, N_{i}^{j+1}\right)$ on the set $E_{i}^{j+1}=E_{i}^{j} \backslash\left\{v_{i}^{j}\right\}$, and the sets $\left(A_{i}^{j+1}, B_{i}^{j+1}\right)$, or finish the induction and define $n=j$.

Suppose $j$ is an even number. If $v_{i}^{j-1} \notin E_{t}$ for any $t \in T$, or if $v_{i}^{j-1} \in E_{t}$ for some $t \in T$ such that $t>t_{j-1}$ in which $v_{i}^{j-1} \in E_{t_{j-1}}^{\prime}$, then let

$$
n=j .
$$

Otherwise, $v_{i}^{j-1} \in E_{t_{j}}$ for some $t_{j} \in T$ such that $t_{j}<t_{j-1}$. Then by the lemma 5.4.5, there exists some $v_{i}^{j} \in E_{t_{j}} \backslash\left\{v_{i}^{j-1}\right\}$ such that $K_{i}$ is also a maximal critical set for $\left(M_{i}^{j} \backslash\left\{v_{i}^{j}\right\}, N_{i}^{j} \backslash\left\{v_{i}^{j}\right\}\right)$ and $v_{i}^{j} \notin \mathrm{cl}_{N_{i}^{j}}\left(K_{i}\right)$. Then let

$$
\begin{aligned}
\left(A_{i}^{j+1}, B_{i}^{j+1}\right) & =\left(A_{i}^{j}, B_{i}^{j} \cup\left\{v_{i}^{j}\right\}\right) \\
\left(M_{i}^{j+1}, N_{i}^{j+1}\right) & =\left(M_{i}^{j} \backslash\left\{v_{i}^{j}\right\}, N_{i}^{j} /\left\{v_{i}^{j}\right\}\right) .
\end{aligned}
$$

Since $\left(M_{i}^{j}, N_{i}^{j}\right)$ has no blockage, by the lemma 5.4.3, $\left(M_{i}^{j+1}, N_{i}^{j+1}\right)$ also has no blockage. 
We claim that for some $j$ this induction stops and $n=j$. Suppose, for a contradiction, that the induction does not stop. Then we get an infinite decreasing sequence

$$
t_{1}>t_{2}>\ldots>t_{j-1}>t_{j}>\ldots
$$

of elements of $\mathbb{N}$ which is a contradiction. Therefore there exists some $n<t_{1}$ such that

$$
V_{i}=\left\{v_{i}^{0}, v_{i}^{1}, v_{i}^{2}, \ldots, v_{i}^{n-1}\right\}
$$

Now, suppose $j$ is an odd number. If $v_{i}^{j-1} \notin E_{s}^{\prime}$ for any $s \in T$, or if $v_{i}^{j-1} \in E_{s}^{\prime}$ for some $s \in T$ such that $s>s_{j-1}$ in which $v_{i}^{j-1} \in E_{s_{j-1}}$, then let

$$
n=j
$$

Otherwise, $v_{i}^{j-1} \in E_{s_{j}}^{\prime}$ for some $s_{j} \in T$ such that $s_{j}<s_{j-1}$. Then by the lemma 5.4.4, there exists some $v_{i}^{j} \in E_{s_{j}}^{\prime} \backslash\left\{v_{i}^{j-1}\right\}$ such that $K_{i}$ is also a maximal critical set for $\left(M_{i}^{j} \backslash\left\{v_{i}^{j}\right\}, N_{i}^{j} \backslash\left\{v_{i}^{j}\right\}\right)$ and $v_{i}^{j} \notin \mathrm{cl}_{M_{i}^{j}}\left(K_{i}\right)$. Then let

$$
\begin{aligned}
\left(A_{i}^{j+1}, B_{i}^{j+1}\right) & =\left(A_{i}^{j} \cup\left\{v_{i}^{j}\right\}, B_{i}^{j}\right) \\
\left(M_{i}^{j+1}, N_{i}^{j+1}\right) & =\left(M_{i}^{j} /\left\{v_{i}^{j}\right\}, N_{i}^{j} \backslash\left\{v_{i}^{j}\right\}\right)
\end{aligned}
$$

Since $\left(M_{i}^{j}, N_{i}^{j}\right)$ has no blockage, by the lemma 5.4.3, $\left(M_{i}^{j+1}, N_{i}^{j+1}\right)$ also has no blockage. We claim that for some $j$ we will stop the induction and $n=j$. Suppose, for a contradiction, that the induction does not stop. Then we get an infinite decreasing sequence

$$
s_{1}>s_{2}>\ldots>s_{j-1}>s_{j}>\ldots
$$

of elements of $\mathbb{N}$ with $s_{1}=t_{1}$ which is a contradiction. Therefore there exists some $n<t_{1}$ such 
that

$$
V_{i}=\left\{v_{i}^{0}, v_{i}^{1}, v_{i}^{2}, \ldots, v_{i}^{n-1}\right\}
$$

Now, we let

$$
\begin{aligned}
\left(A_{i+1}, B_{i+1}\right) & =\left(A_{i}^{n}, B_{i}^{n}\right) \\
\left(M_{i+1}, N_{i+1}\right) & =\left(M_{i}^{n}, N_{i}^{n}\right) \\
E_{i+1} & =E_{i} \backslash V_{i} .
\end{aligned}
$$

Now, assume that we have the [Case2] that $e_{i} \in \mathrm{cl}_{N_{i}}\left(K_{i}\right)$. Since $\left(M_{i}, N_{i}\right)$ has no blockage, we have $e_{i} \notin \mathrm{cl}_{M_{i}}\left(K_{i}\right)$. Similarly as in the [Case1], we can construct a finite subset

$$
V_{i}=\left\{v_{i}^{0}, v_{i}^{1}, v_{i}^{2}, \ldots, v_{i}^{n-1}\right\}
$$

of $E$ such that $v_{i}^{0}=e_{i}$ and

$$
A_{i+1} \cup B_{i+1}=A_{i} \cup B_{i} \cup V_{i}
$$

and a pair of matroids $\left(M_{i}^{j}, N_{i}^{j}\right)$ on the set $E_{i}^{j}=E_{i} \backslash\left\{v_{i}^{0}, v_{i}^{1}, v_{i}^{2}, \ldots, v_{i}^{j-1}\right\}$ for each $1 \leq j \leq n$ such that it has no blockage and a pair $\left(A_{i}^{j}, B_{i}^{j}\right)$ of finite disjoint subsets of $E$. The difference is that

$$
\begin{aligned}
\left(A_{i}^{1}, B_{i}^{1}\right) & =\left(A_{i} \cup\left\{v_{i}^{0}\right\}, B_{i}\right), \\
\left(M_{i}^{1}, N_{i}^{1}\right) & =\left(M_{i} /\left\{v_{i}^{0}\right\}, N_{i} \backslash\left\{v_{i}^{0}\right\}\right) .
\end{aligned}
$$

Then, we let

$$
\begin{aligned}
\left(A_{i+1}, B_{i+1}\right) & =\left(A_{i}^{n}, B_{i}^{n}\right) \\
\left(M_{i+1}, N_{i+1}\right) & =\left(M_{i}^{n}, N_{i}^{n}\right) \\
E_{i+1} & =E_{i} \backslash V_{i} .
\end{aligned}
$$

Now, let $A=\bigcup_{i \in \mathbb{N}} A_{i}$ and $B=\bigcup_{i \in \mathbb{N}} B_{i}$. Observe that $(A, B)$ is a pair of disjoint subsets of $E$ because for each $i \in \mathbb{N}$ we have $\left(A_{i}, B_{i}\right)$ is a pair of disjoint subsets of $E$. We want to show that $(A, B)$ 
is a covering for $(M, N)$. It is enough to show that $(A, B)$ are independent sets in $(M, N)$. We first show that $B$ is independent in $N$. Suppose, for a contradiction, that there is a circuit $C$ of $N$ such that $C \subseteq B$.

First assume that $C$ is a finite circuit. Let enumerate the circuit $C=\left\{e_{c_{1}}, e_{c_{2}}, \ldots, e_{c_{m}}\right\}$ such that each $c_{i} \in \mathbb{N}$ and $c_{i}<c_{j}$ if and only if $i<j$ for the $1 \leq i, j \leq m$. Let $p \in \mathbb{N}$ be that such that $e_{p}=e_{c_{m}}$. Then, $\left\{e_{p}\right\}$ is a loop in the matroid $N_{p}$. Let $K_{p} \subseteq E_{p} \backslash\left\{e_{p}\right\}$ be a maximal critical set for $\left(M_{p} \backslash\left\{e_{p}\right\}, N_{p} \backslash\left\{e_{p}\right\}\right)$. Since $\left\{e_{p}\right\}$ is a loop of $N_{p}$ we have $e_{p} \in \mathrm{cl}_{N_{p}}\left(K_{p}\right)$. On the other side, we know that $\left(M_{p}, N_{p}\right)$ has no blockage. This implies that $e_{p} \notin \mathrm{cl}_{M_{p}}\left(K_{p}\right)$. So $e_{p} \in A_{p}^{1}$ which implies that $e_{p} \in A$. So, we have $e_{p} \in C \cap A$ which is a contradiction with the assumption that $C \subseteq B$.

Now, assume that $C$ is an infinite circuit. Let $i \in \mathbb{N}$ be such that $e_{i} \in C$ and $i<j$ for any $e_{j} \in C$. Consider the subset $\left\{e_{1}, e_{2}, \ldots, e_{i-1}\right\}$ of $E$ and the family of sets $\left\{V_{1}, V_{2}, \ldots, V_{i-1}\right\}$. Since the set $V_{\alpha}$ is finite for each $1 \leq m \leq i-1$, we have

$$
\bigcup_{\alpha=1}^{i-1} V_{\alpha}
$$

is finite. Therefore

$$
C \cap \bigcup_{\alpha=1}^{i-1} V_{\alpha}
$$

is finite. Let $j \in \mathbb{N}$ be the smallest index such that

$$
e_{j} \in C \backslash \bigcup_{\alpha=1}^{i-1} V_{\alpha}
$$

Since $e_{j} \notin \bigcup_{\alpha=1}^{i-1} V_{\alpha}$, we have $e_{j} \notin A_{j} \cup B_{j}$. Let the set $K_{j} \subseteq E_{j} \backslash\left\{e_{j}\right\}$ be a maximal critical set for

$$
\left(M_{j} \backslash\left\{e_{j}\right\}, N_{j} \backslash\left\{e_{j}\right\}\right)
$$

Since $e_{j} \in B$ we have $e_{j} \notin \mathrm{cl}_{N_{j}}\left(K_{j}\right)$. So, there exists some $v_{j}^{1} \in C \backslash\left\{v_{j}^{0}\right\}$ such that $v_{j}^{1} \in A_{j}^{2}$. This implies that $v_{j}^{1} \in A$. So, we have $v_{j}^{1} \in C \cap A$ which is a contradiction with $C \subseteq B$. This 
completes the proof that $B$ is independent in $N$. By a similar argument, it can be shown that $A$ is independent in $M$. Thus, $(A, B)$ is a covering for $(M, N)$.

Now we are ready to conclude the the Matroid Intersection Conjecture for singular matroids on an infinite countable set.

Proof of Corollary 5.1.5. Since $M$ is singular if and only if $M^{*}$ is singular, it is enough to show that $M^{*}$ and $N$ satisfies the Matroid Intersection Conjecture. Since matroids $M$ and $N$ are singular on an infinite countable set $E$, by Theorem 5.1.4, we have the followings are equivalent:

1. $(M, N)$ has a covering.

2. $(M, N)$ has no blockage.

Now, by Theorem 5.1.2, we have $(M, N)$ has the Packing/Covering Property. Finally, by Theorem 3.1.3, we have $\left(M^{*}, N\right)$ satisfies the Matroid Intersection Conjecture. 


\section{Chapter 6}

\section{Conclusion and Future Work}

\subsection{Summary}

For this chapter, we assume that $M$ and $N$ are matroids on a common ground set $E$. In this section, we summarize the main results of this dissertation that imply the Matroid Intersection Conjecture 1.2.1.

Theorem. 3.1.9. If $(M, N)$ has the Almost Intersection Property, then it satisfies the Matroid Intersection Conjecture.

Theorem. 5.1.2. If the followings are equivalent:

1. $(M, N)$ has a covering.

2. $(M, N)$ has no blockage.

than $(M, N)$ has the Packing/Covering Property.

We proved that the Matroid Intersection Conjecture 1.2.1 is true for $(M, N)$ in the following cases:

- $M$ has finite rank and $N$ is arbitrary (Section 3.4).

- $M$ is patchwork and $N$ is arbitrary (Section 3.4). 
- $M$ and $N$ are singular matroids on an infinite countable set $E$ (Section 5.4).

We also provided a new proof that the Matroid Intersection Conjecture 1.2.1 is true for $(M, N)$ in the following case:

- $M$ and the dual of $N$ are nearly finitary matroids (Section 3.4).

\subsection{Statements Equivalent to the Matroid Intersection Con- jecture}

In this section, we summarize the conjectures related to this dissertation. We first state the conjectures that are equivalent to the Matroid Intersection Conjecture. Then, in Corollary 6.2.3, we provide more statements equivalent to the Packing/Covering Conjecture.

The equivalency of the following conjectures is proved in [12].

The Matroid Intersection Conjecture: Any two matroids $M$ and $N$ on a common set $E$ have a common independent set $I$ admitting a partition $I=J_{M} \sqcup J_{N}$ such that $\operatorname{cl}_{M}\left(J_{M}\right) \cup \operatorname{cl}_{N}\left(J_{N}\right)=E$.

The pairwise Packing/Covering Conjecture: Any pair of matroids on the same ground set has the Packing/Covering property.

The Packing/Covering Conjecture: Any family of matroids on the same ground set has the Packing/Covering property.

The Packing Conjecture: A family of matroids $\left(M_{k}: k \in K\right)$ on the same ground set $E$ has a packing if and only if the following condition is true for every $Y \subseteq E$ :

If $\left(M_{k} \cdot Y: k \in K\right)$ has a covering, then it also has a packing.

The Covering Conjecture: A family of matroids $\left(M_{k}: k \in K\right)$ on the same ground set $E$ has a covering if and only if the following condition is true for every $Y \subseteq E$ :

If $\left(M_{k} \uparrow_{Y}: k \in K\right)$ has a packing, then it also has a covering.

Remark 6.2.1. Here, we sum up the cases for which the pairwise Packing/Covering conjecture is known to be true: 
1. When both matroids are finitary [7].

2. When both matroids are nearly finitary [7].

3. When one matroid is finitary the other is a countable direct sum of matroids whose duals are of finite rank [5].

4. When both matroids has only countably many circuits [12].

5. When one matroid is $M_{F C}(G)$ and the other is $M_{T C}(G)$ for a locally finite graph $G$ with a tree-decomposition into finite parts of adhesion at most 2 [11].

6. When one matroid is $M_{\Psi_{1}}(G)$ and the other is $M_{\Psi_{2}}(G)$ for a a locally finite graph $G$ with a tree-decomposition into finite parts of adhesion at most 2 where $\Psi_{1}$ and $\Psi_{2}$ are Borel sets of ends of $G[11]$.

7. When one is a finite co-rank matroid and the other is arbitrary (Section 3.4).

8. When one is a patchwork matroid and the other is arbitrary (Section 3.4).

9. When both matroids are singular on a countable ground set (Section 5.4).

Definition 6.2.2. Let $\mathscr{M}=\left(M_{k}: k \in K\right)$ be a family of matroids on $P$ and $P \subseteq E$. A hindrance for $\mathscr{M}$ on $P$ is a packing $\left(S_{k}: k \in K\right)$ of $\mathscr{M} \uparrow_{P}=\left(M_{k} \uparrow_{P}: k \in K\right)$ with $\bigcup_{k \in K} S_{k} \neq P$, that is, it is a packing of $\mathscr{M} \uparrow_{P}$ that is not a covering. An obstruction for $\mathscr{M}$ on $P$ is a packing of $\mathscr{M} \uparrow_{P}$ such that $\mathscr{M} \uparrow_{P}$ has no covering. The family $\mathscr{M}$ is called unhindered if and only if it has no hindrance (on any $P \subseteq E$ ) and unobstructed if and only if it has no obstruction (on any $P \subseteq E$ ).

In the following, we provide more statements that are all equivalent to the Packing/Covering conjecture.

Corollary 6.2.3. Let $\mathscr{A}$ be a class of matroids closed under contractions (in particular, it can be the class of all matroids). The following conditions are equivalent: 
1. For every family $\mathscr{M}$ of matroids from $\mathscr{A}$ on the same set, $\mathscr{M}$ is unobstructed if and only if it has a covering.

2. For every family $\mathscr{M}$ of matroids from $\mathscr{A}$ on the same set, if $\mathscr{M}$ is unobstructed then it has a covering.

3. For every family $\mathscr{M}$ of matroids from $\mathscr{A}$ on the same set, if $\mathscr{M}$ is unhindered then it has a covering.

4. For every family $\mathscr{M}$ of matroids from $\mathscr{A}$ on the same set, if $\mathscr{M}$ is loose then it has a covering.

5. Every family of matroids from $\mathscr{A}$ on the same set has the Packing/Covering property.

Proof. Clearly (1) implies (2), (2) implies (3), and (3) implies (4).

To see that (4) implies (5), let $\mathscr{M} \subseteq \mathscr{A}$ be a family of matroids on $E$. There is $P \subseteq E$ such that $\mathscr{M} \uparrow_{P}$ has a packing and $\mathscr{M}$.C is loose (where $C=E \backslash P$ ). Since $\mathscr{A}$ is closed under contractions, by (4) $\mathscr{M} . C$ has a covering.

To see that (5) implies (1), let $\mathscr{M}$ be unobstructed and $E=P \sqcup C$ be a partition such that $\mathscr{M} \uparrow_{P}$ has a packing and $\mathscr{M} . C$ has a covering. Since $\mathscr{M}$ is unobstructed, the family $\mathscr{M} \uparrow_{P}$ has a covering. If $\left(A_{i}: i \in I\right)$ is a covering of $\mathscr{M} \uparrow_{P}$ and $\left(A_{i}^{\prime}: i \in I\right)$ is a covering of $\mathscr{M}$.C, then $\left(A_{i} \cup A_{i}^{\prime}: i \in I\right)$ is a covering of $\mathscr{M}$.

\subsection{Future Work}

We are certain that we can use our tools and results in Chapter 4 to attack the Matroid Intersection Conjecture 1.2.1 for a more general family of matroids. We propose the following definition and conjecture.

Definition 6.3.1. Let $M=\bigoplus_{i \in I} M_{i}$ be a matroid corresponding to the partition $E=\bigsqcup_{i \in I} E_{i}$ with each $M_{i}$ is either a uniform matroid of rank finite or a uniform matroid co-rank finite on the 
set $E_{i}$, and $N=\bigoplus_{j \in J} N_{j}$ corresponding to the partition $E=\bigsqcup_{j \in J} E_{j}^{\prime}$ with each $N_{j}$ is either a uniform matroid of rank finite or a uniform matroid co-rank finite on the set $E_{j}^{\prime}$. We call such matroids $M$ and $N$ strong.

Conjecture 6.3.2. If $M$ and $N$ are strong on an infinite countable set $E$, then the followings are equivalent:

1. $(M, N)$ has a covering.

2. $(M, N)$ has no blockage.

If we prove that this conjecture is true, then by Theorem 5.1.2 we can prove that the Matroid Intersection Conjecture is true for strong matroids. 


\section{Bibliography}

[1] R. Aharoni, On a duality principle in infinite bipartite graphs, J. Lond. Math. Soc. (2), 28 (1983), 385-392.

[2] R. Aharoni, König's duality theorem for infinite bipartite graphs, J. Lond. Math. Soc. 29 (1984), 1-12.

[3] R. Aharoni , C. St. J. A. Nash-Williams, and S. Shelah, A general criterion for the existence of transversals, Proc. Lond. Math. Soc. 47 (1983), 43-68.

[4] R. Aharoni and C. Thomassen, Infinite, highly connected digraphs with no two arcdisjoint spanning trees, J. Graph Theory, 13 (1989) 71-74.

[5] R. Aharoni and R. Ziv, The intersection of two infinite matroids, J. Lond. Math. Soc. 58 (1998), 513-525.

[6] E. Aigner-Horev, J. Carmesin, and J. Fröhlich, Infinite matroid union I, preprint arXiv:1111.0602 (2011).

[7] E. Aigner-Horev, J. Carmesin, and J. Fröhlich, On the intersection of infinite matroids, preprint arXiv:1111.0606 (2011).

[8] D.W.T. Bean, A connected finitary co-finitary matroid is finite, Proceedings of the Seventh Southeastern Conference on Combinatorics, Graph Theory and Computing, Congressus Numerantium 17 (1976), 115-19. 
[9] N. Bowler and J. Carmesin, An excluded minors method for infinite matroids, preprint arXiv:1212.3939 (2013).

[10] N. Bowler and J. Carmesin, Matroids with an infinite circuit-cocircuit intersection, J. Combin. Theory, Ser. B 107 (2014), 78-91.

[11] N. Bowler and J. Carmesin, On the intersection conjecture for infinite trees of matroids, preprint arXiv:1404.6067 (2014).

[12] N. Bowler and J. Carmesin, Matroid intersection, base packing and base covering for infinite matroids, Combinatorica 35 (2015), no. 2, 153-180.

[13] N. Bowler and J. Carmesin, private communication.

[14] N. Bowler, J. Carmesin, S. Ghaderi, and J. Wojciechowski, The almost intersection property for pairs of matroids on common ground set, in preparation.

[15] N. Bowler and St. Geschke, Self-dual uniform matroids on infinite sets, Proc. Amer. Math. Soc. 144 (2015), no. 2, 459-471.

[16] N. Bowler and M. Storm, Patchwork matroids, in preparation.

[17] R. A. Brualdi, Strong transfinite version of König's duality theorem, Monatsh. Math., 75 (1971), 106-110.

[18] H. Bruhn and R. Diestel, Infinite matroids in graphs, Discrete Math. 311 (2011), $1461-1471$.

[19] H. Bruhn, R. Diestel, M. Kriesell, R. Pendavingh, and P. Wollan, Axioms for infinite matroids, Adv. Math., 239 (2013), 18-46.

[20] H. Bruhn and P. Wollan, Finite connectivity in infinite matroids, European J. Comb. 33 (2012), 1900-1912. 
[21] R. Diestel, Graph Theory (4th edition), Springer, 2010. Electronic edition available at: http://diestel-graph-theory.com/index.html

[22] J. Edmonds, Submodular functions, matroids, and certain polyhedra, Combinatorial structures and their applications, Proc. Calgary Internat. Conf. (1970), 69-87.

[23] S. Ghaderi and J. Wojciechowski, Matroid intersection for singular matroids, in preparation.

[24] P. Hall, On representatives of subsets, J. Lond. Math. Soc. 10 (1935), 26-30.

[25] D. Higgs, Equicardinality of bases in B-matroids, Canad. Math. Bull. 12 (1969), 861-862.

[26] D. Higgs, Infinite graphs and matroids, Recent Progress in Combinatorics, Proceedings Third Waterloo Conference on Combinatorics, Academic Press, 1969, 245-53.

[27] D. Higgs, Matroids and duality, Colloq. Math. 20 (1969), 21-220.

[28] M. Holz, K.-P. Podewski, K. Steffens, Injective Choice Functions, Springer, 1987.

[29] M. Las Vergnas, Sur la dualité en théorie des matröids, Théorie des Matröids, Lecture notes in mathematics, vol. 211, Springer, 1971, 67-85.

[30] H. Nishimura and S. Kuroda, A lost mathematician, Takeo Nakasawa, The forgotten father of matroid theory, Birkhäuser, 2009.

[31] J. Oxley, Infinite matroids, Proc. Lond. Math. Soc. (3) 37 (1978), no. 2, 259-272.

[32] J. Oxley, Matroid Theory, Oxford University Press, 1992.

[33] K.-P. Podewski and K. Steffens, Injective choice functions for countable families, J. Combin. Theory. Ser. B, 21 (1976), 40-46.

[34] R. Rado, Abstract linear dependence, Colloq. Math. 14 (1966), 257-264. 
[35] W. Sierpiński, Sur les espaces (V) de M. Fréchet dense en soi, Fund. Math. 33 (1945), 174-176.

[36] W. Sierpiński, General Topology, Oxford University Press, 1956.

[37] K. Steffens, Injective choice functions, J. Combin. Theory, Ser. A, 17 (1974), 138-144.

[38] H. Whitney, On the abstract properties of linear dependence, Amer. J. Math. 57 (1935) no. 3, 509-533.

[39] J. Wojciechowski, Infinite matroidal version of Hall's matching theorem, J. Lond. Math. Soc. 71 (2005), 563-578. 